\title{
WestVirginiaUniversity
}

THE RESEARCH REPOSITORY @ WVU

Graduate Theses, Dissertations, and Problem Reports

2002

\section{Aging model for commercial polymers}

\author{
Kevin J. Ford \\ West Virginia University
}

Follow this and additional works at: https://researchrepository.wvu.edu/etd

\section{Recommended Citation}

Ford, Kevin J., "Aging model for commercial polymers" (2002). Graduate Theses, Dissertations, and Problem Reports. 1410.

https://researchrepository.wvu.edu/etd/1410

This Thesis is protected by copyright and/or related rights. It has been brought to you by the The Research Repository @WVU with permission from the rights-holder(s). You are free to use this Thesis in any way that is permitted by the copyright and related rights legislation that applies to your use. For other uses you must obtain permission from the rights-holder(s) directly, unless additional rights are indicated by a Creative Commons license in the record and/ or on the work itself. This Thesis has been accepted for inclusion in WVU Graduate Theses, Dissertations, and Problem Reports collection by an authorized administrator of The Research Repository @ WVU. For more information, please contact researchrepository@mail.wvu.edu. 


\section{Aging Model for Commercial Polymers}

\section{Kevin J. Ford}

Thesis submitted to the College of Engineering and Mineral Resources

at West Virginia University in partial fulfillment of the requirements

for the degree of

Master of Science

in

Mechanical Engineering

Ever J. Barbero, Ph.D., Chair

Wade W. Huebsch, Ph.D.

Jacky Prucz, Ph.D.

Department of Mechanical and Aerospace Engineering

Morgantown, West Virginia

2002

Keywords: creep, time-temperature superposition principle, polyvinyl chloride, thermoset, thermoplastic 


\title{
ABSTRACT
}

\section{Aging of Commercial Polymers}

\begin{abstract}
Kevin J. Ford
Physical aging of polyvinyl chloride, high-density polyethylene, and polyester reinforced with polyester fibers specimens have been investigated. These materials are commercially available and are currently used in trenchless relining of sewer pipes. The analysis uses the effective-time theory and time-temperature superposition (TTSP) methods proposed by Struik, as well as variations of these methods, as proposed by Chai and McCrum, Guerdoux et al. and others. The creep data generated by this study show that the time-dependant creep properties of these materials can be modeled within experimental error with slight changes to the above methods. To predict the creep behavior of full-scale specimens; these properties were then shifted from their testing age and temperature to the testing age and temperature of the full-scale specimens. It was shown that a rotation, along with a horizontal shift of the aging data, is needed to obtain the ageing parameter of the materials.
\end{abstract}




\section{DEDICATION}

This thesis is dedicated to my grandparents, the late John and Anna Kondik and the late Ross and Martha Ford. Without their influence in my life this would not have been possible, thanks. 


\section{ACKNOWLEDGEMENTS}

I would like to thank Dr. John Loth for encouraging me to pursue my master's degree and advising me throughout my time at West Virginia University. Additionally I would like to thank all of the professors in the Mechanical and Aerospace Engineering Department at West Virginia University who suffered through the difficult process of teaching me as an undergraduate and graduate student.

I would like to thank Dr. Ever Barbero for hiring me and being my advisor on this project and encouraging me to stay for my Ph.D. I would also like to thank Dr. Jacky Prucz and Dr. Wade Huebsch for serving on my committee. Much thanks goes to the National Science Foundation and the Pipe Rehabilitation Council for funding this project. I would also like to thank Shalini Rangarajan, Michael Julius, and Edward Wen for their help on this project. Thanks to Chuck Coleman for helping me with the wiring of the optical encoders, Clifford Judy for teaching and helping me in the machine shop, and Lee Metheney for his help with problems in the lab.

Thanks to all of my friends who supported me throughout my time in college. I would also like to thank my family for helping me get through college and for always being there for me, no matter how big of pain I was. Lastly, if I forgot to mention anyone forgive me and write your name in the space below: 


\section{TABLE OF CONTENTS}

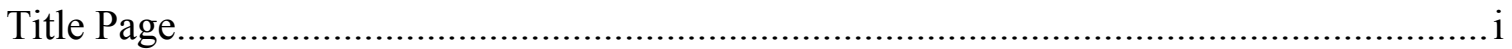

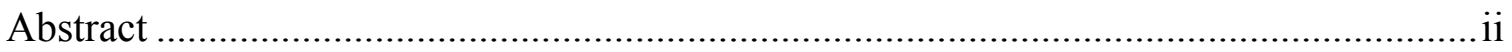

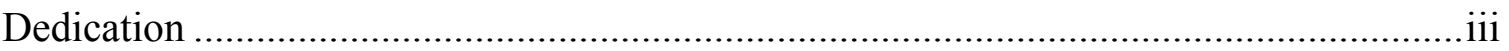

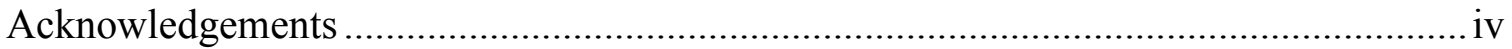

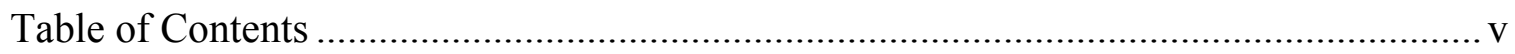

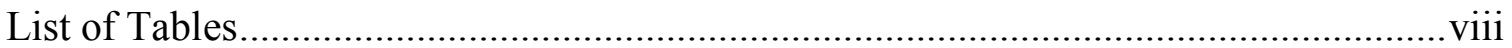

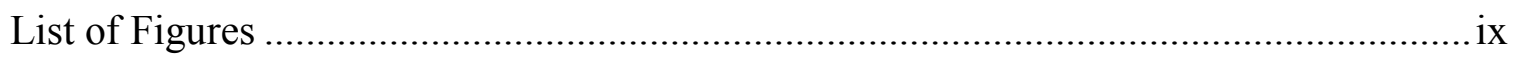

Chapter 1 Introduction and Literature Review...................................................... 1

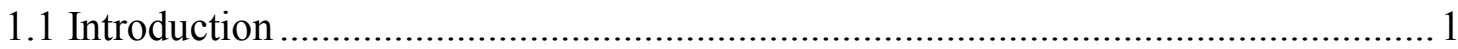

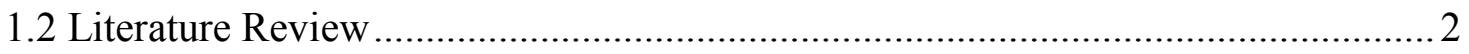

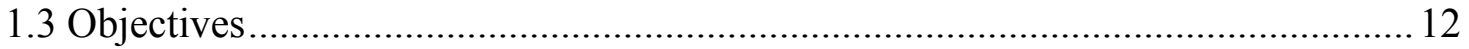

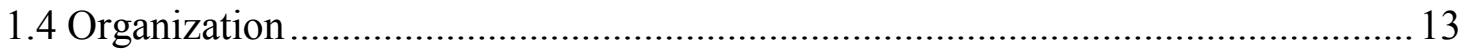

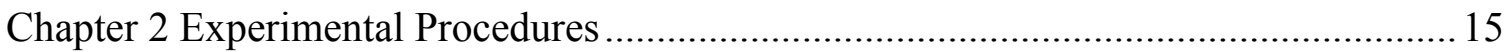

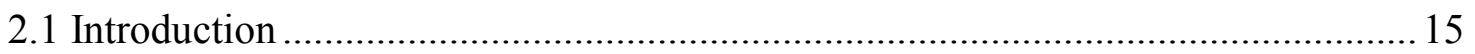

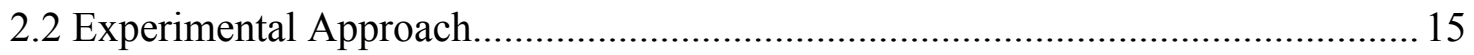

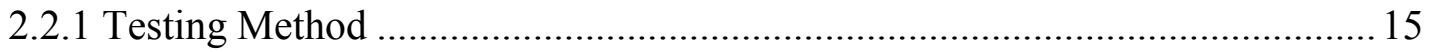

2.2.2 Design and Fabrication of Testing Apparatus ............................................ 15

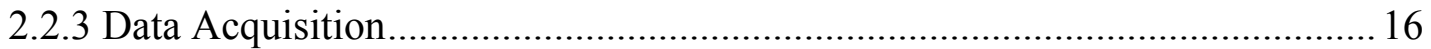

2.3 Test Environmental Conditions................................................................... 17

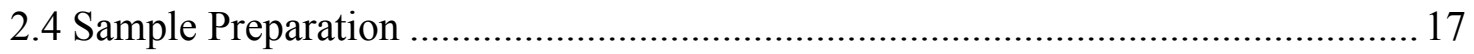

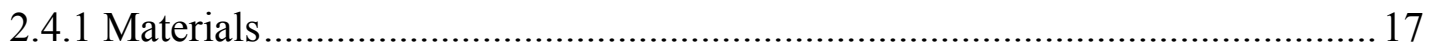

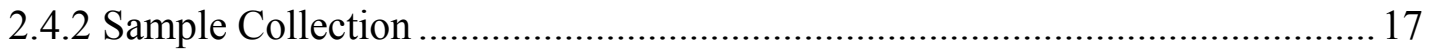




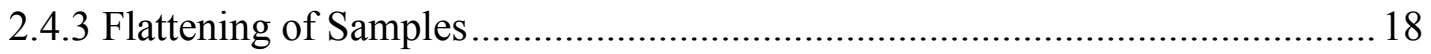

2.4.4 Sample Sizing and Preparation .................................................................. 18

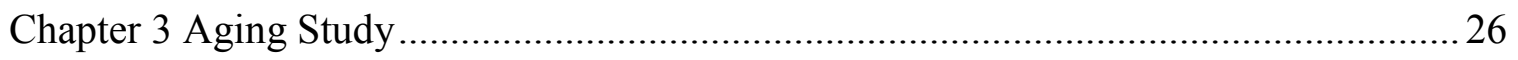

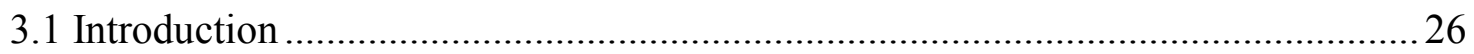

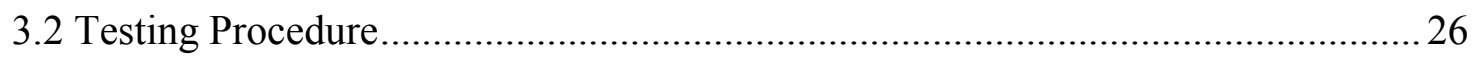

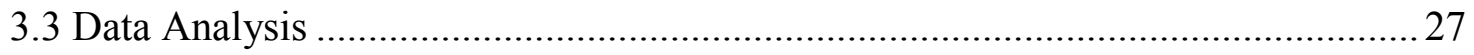

Chapter 4 Time-Temperature Superposition Principle ……….......................................... 45

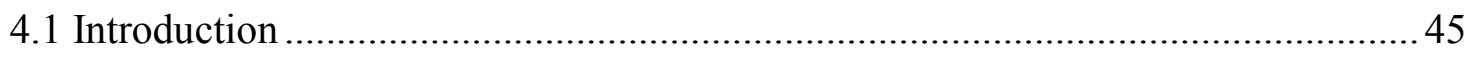

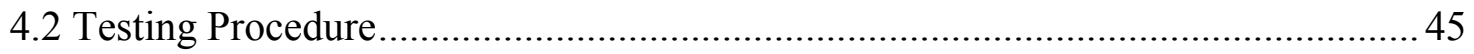

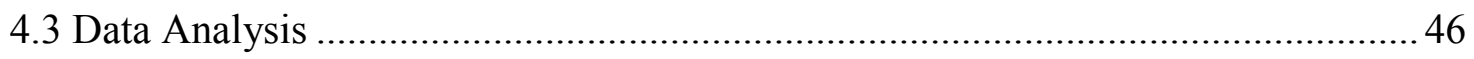

Chapter 5 Long-Term Testing and Correlation with Full Size Specimens ........................53

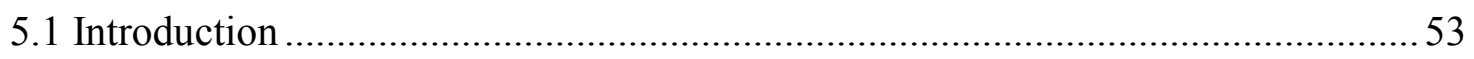

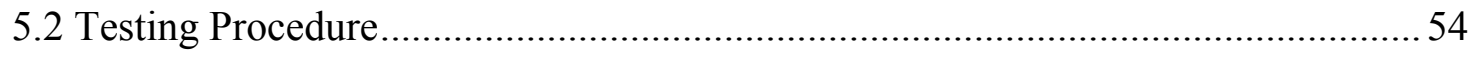

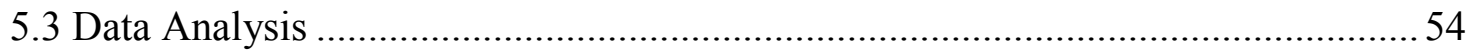

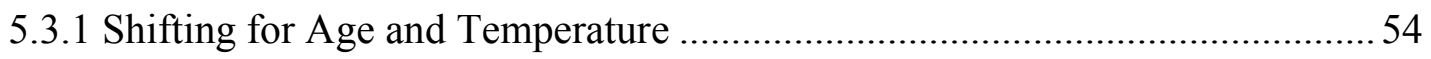

5.3.2 Calculating Ageing Shift Factors ....................................................................5

5.3.3 Calculating Temperature Shift Factors ..........................................................56

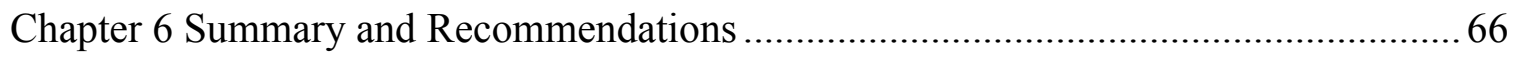

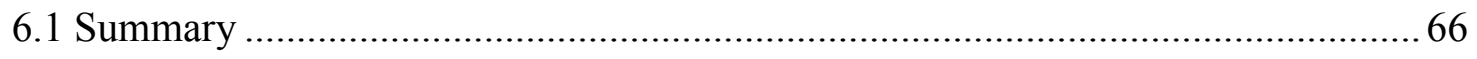

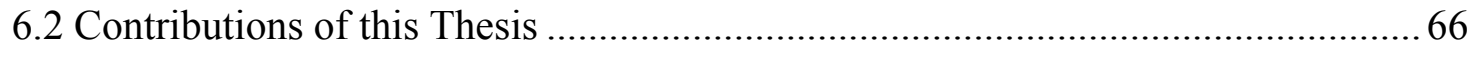

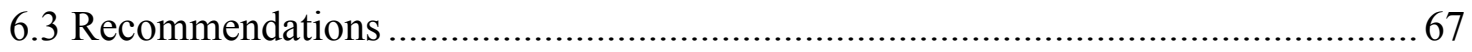

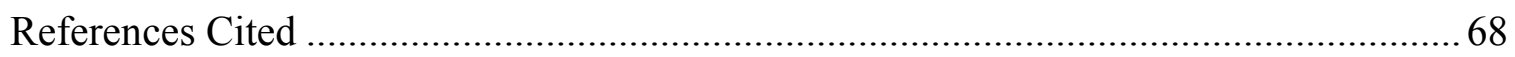

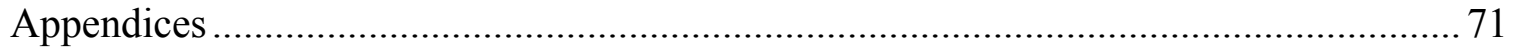


Appendix A - Drawing Package of Testing Apparatus .......................................... 71

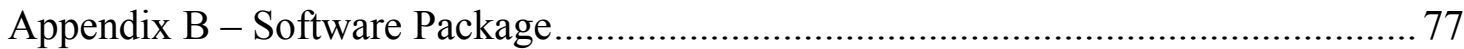




\section{LIST OF TABLES}

Table 2.1. Material Properties................................................................................. 20

Table 3.1. Typical testing schedule for an aging study .................................................. 33

Table 3.2. Comparison of aging studies of material A......................................................33

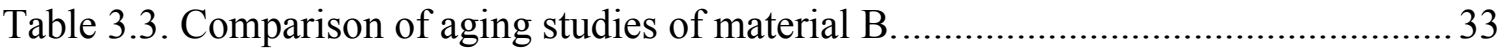

Table 3.4. Comparison of aging studies of material C.................................................... 34

Table 3.5. Comparison of aging studies of material D....................................................... 34

Table 3.6. Comparison of aging studies of material E...................................................... 34

Table 3.7. Comparison of master $\mu$ at $21.1{ }^{\circ} \mathrm{C}$ and $40{ }^{\circ} \mathrm{C}$ for material A............................35

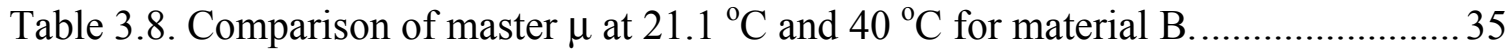

Table 3.9. Comparison of master $\mu$ at $21.1{ }^{\circ} \mathrm{C}$ and $40{ }^{\circ} \mathrm{C}$ for material C............................35

Table 5.1. Start date for testing of full-scale pipes......................................................5 


\section{LIST OF FIGURES}

Figure 1.1. Illustration of trenchless lining of a pipe. ................................................. 14

Figure 1.2. Idealized temperature scan of a polymer [Fig. 5.4, 12] ............................... 14

Figure 2.1. Illustration of the location from which the samples are removed...................20

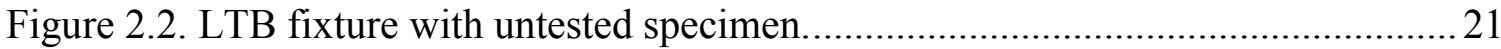

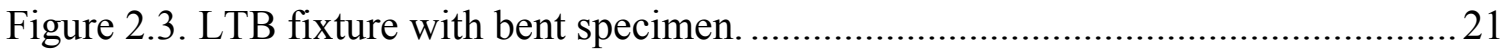

Figure 2.4. LTB fixtures in environmental chamber under testing................................. 22

Figure 2.5. Specimen held in place with rubber bands. ...............................................22

Figure 2.6. Illustration of specimen under four-point bending, the dashed line represents

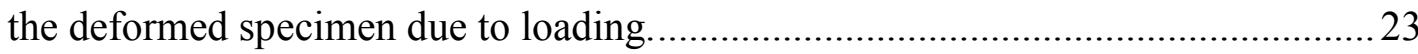

Figure 2.7. Computers used for data acquisition............................................................... 23

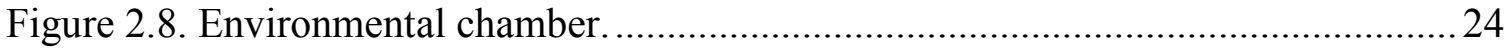

Figure 2.9. Illustration of one-third of pipe between two plates for flattening purposes. . 24

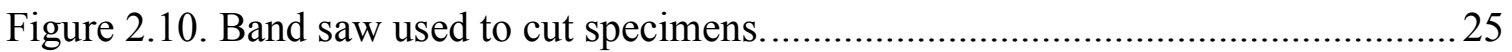

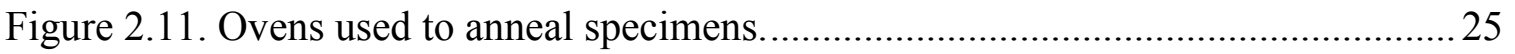

Figure 3.1. Deflection versus time for aging study test. ................................................... 36

Figure 3.2. Illustration of the reasoning for mechanical conditioning..............................36

Figure 3.3. Fit of the data using the Modified Power Law............................................. 37

Figure 3.4. Aging study data transformed into the Power Law for material A................. 37

Figure 3.5. Aging study data transformed into the Power Law for material B.................38

Figure 3.6. Aging study data transformed into the Power Law for material C................. 38

Figure 3.7. Aging study data transformed into the Power Law for material D................. 39

Figure 3.8. Aging study data transformed into the Power Law for material E................ 39 
Figure 3.9. Aging study data shifted to obtain $\mu$ for material A. ................................... 40

Figure 3.10. Aging study data shifted to obtain $\mu$ for material B. .................................. 40

Figure 3.11. Aging study data shifted to obtain $\mu$ for material C. ............................... 41

Figure 3.12. Aging study data shifted to obtain $\mu$ for material D............................... 41

Figure 3.13. Aging study data shifted to obtain $\mu$ for material E................................. 42

Figure 3.14. Theoretical and experimental values of the aging shift factors for material A

Figure 3.15. Theoretical and experimental values of the aging shift factors for material B.

Figure 3.16. Theoretical and experimental values of the aging study shift factors for material C.

Figure 3.17. Theoretical and experimental values of the aging shift factors for material D.

Figure 3.18. Theoretical and experimental values of the aging shift factors for material E.

Figure 4.1. TTSP graphs and master curve shifted to $21.1{ }^{\circ} \mathrm{C}$ of material A.................. 48

Figure 4.2. TTSP graphs and master curve shifted to $20.27^{\circ} \mathrm{C}$ of material B................ 48

Figure 4.3. TTSP graphs and master curve shifted to $20.28{ }^{\circ} \mathrm{C}$ of material C................ 49

Figure 4.4. TTSP graphs and master curve shifted to $21.9{ }^{\circ} \mathrm{C}$ of material D.................49

Figure 4.5. TTSP graphs and master curve shifted to $40{ }^{\circ} \mathrm{C}$ of material E. ....................50

Figure 4.6. Theoretical and experimental values of the TTSP shift factors for material A. 
Figure 4.7. Theoretical and experimental values of the TTSP shift factors for material B.

Figure 4.8. Theoretical and experimental values of the TTSP shift factors for material C.

Figure 4.9. Theoretical and experimental values of the TTSP shift factors for material D.

Figure 4.10. Theoretical and experimental values of the TTSP shift factors for material E.

Figure 5.1. TTSP and prediction at $40{ }^{\circ} \mathrm{C}$ and $\mathrm{t}_{\mathrm{e}}=1 \mathrm{hr}$ for material A......................58

Figure 5.2. TTSP and prediction at $40{ }^{\circ} \mathrm{C}$ and $\mathrm{t}_{\mathrm{e}}=1 \mathrm{hr}$ for material B......................59

Figure 5.3. TTSP and prediction at $40{ }^{\circ} \mathrm{C}$ and $\mathrm{t}_{\mathrm{e}}=1 \mathrm{hr}$ for material C........................59

Figure 5.4. TTSP and prediction at $40{ }^{\circ} \mathrm{C}$ and $\mathrm{t}_{\mathrm{e}}=1 \mathrm{hr}$ for material D.......................60

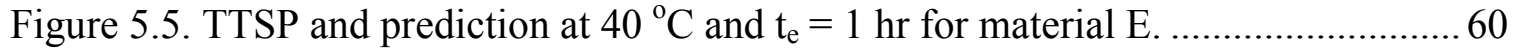

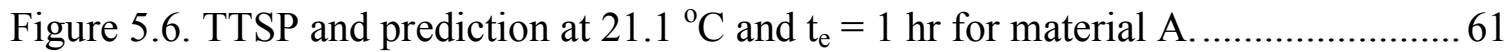

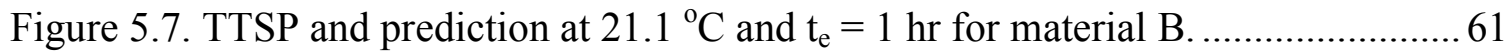

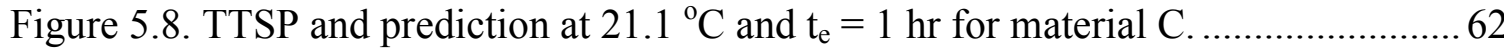

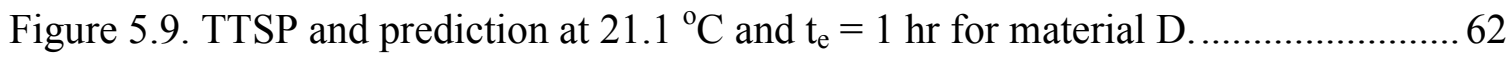

Figure 5.10. Comparison of full-size pipes and prediction for material A. .................... 63

Figure 5.11. Comparison of full-size pipes and prediction for material B.....................63

Figure 5.12. Comparison of full-size pipes and prediction for material C...................... 64

Figure 5.13. Comparison of full-size pipes and prediction for material D. .................... 64

Figure 5.14. Comparison of full-size pipes and prediction for material E..................... 65

Figure A2 Testing fixture load support arm side view.............................................. 72 
Figure A3 Testing fixture load support arm bottom view........................................ 72

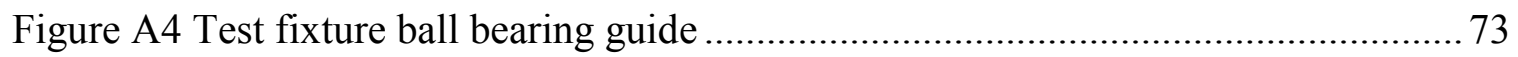

Figure A5 Test fixture carriage assembly top and outside views ............................... 74

Figure A6 Testing fixture carriage assembly inside view ........................................ 75

Figure A7 Testing fixture spar and mirror measuring device ................................... 75

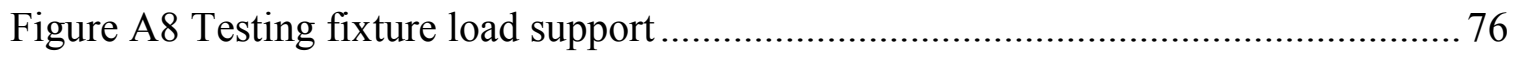

Figure B.1 Screen shot of the form frmSelections, which allows users to enter the filename to write to and select the time interval to write the data. 94

Figure B.2 Screen shot of the from frmF5312 which allows users to zero the system..... 95 


\section{CHAPTER 1 INTRODUCTION AND LITERATURE REVIEW}

\subsection{Introduction}

The Urban Wet-Weather Federal Advisory Committee (FAC) and the Environmental Protection Agency (EPA) are developing a new set of standards and regulations that the current sewer systems in the United States must comply with. These new standards and regulations will require municipalities to invest heavily on the rehabilitation of the collection sewer systems. Due to high costs and traffic disruptions, trenchless lining of existing pipes is being used. In trenchless lining, a polymer or reinforced-polymer is applied to the inside of the existing host-pipe, without disturbing the soil or any aboveground facilities (Fig. 1.1). Several technologies offer various solutions, including thermoplastic and thermoset polymers and composites, which can be cured within the host-pipe or mechanically installed to fit the host pipe. No accepted unbiased testing procedure has yet been established that allows a fair comparison of the various products for a particular field installation.

The main purpose of the liner is to prevent water leakage into the sewer pipe. Therefore, a liner encased by a host sewer pipe is subject to hydrostatic external pressure, but it may also carry some soil pressure if the host pipe is deteriorated severely. Regardless of the source of external pressure, the encased liner fails by creep-buckling under external pressure. If the time-dependent properties of the liner are known, realistic predictions of the buckling pressure of a liner can be done by rigorous numerical modeling of the buckling of a confined pipe. 


\subsection{Literature Review}

There has been a significant amount of work previously conducted that relates to the prediction of time-dependent properties of materials, particularly PVCs and composite materials. This literature review discusses the relevant studies to this project.

Lakes [1] describes a viscoelastic material as those for which the relationship between stress and strain depends on time. He further explains that some phenomena of viscoelastic materials are: 1) if the stress is held constant, the strain increases with time (creep) and 2) if the strain is held constant, the stress decreases with time (relaxation). He defines the creep compliance as

$$
J(t)=\frac{\varepsilon(t)}{\sigma_{o}}
$$

where $\mathrm{J}$ is the torsional compliance, $\varepsilon(\mathrm{t})$ is the strain as a function of time, and $\sigma$ is stress.

Struik [2] has shown that creep curves of quenched polymers are nearly horizontally shifted towards longer times with increase in aging time. He postulates that the retardation spectrum is shifted without any modification during aging. Furthermore, he postulates that the $\log \left(t_{\mathrm{e}}\right)$ vs. $\log \left(\mathrm{a}_{\mathrm{e}}\right)$ plot is linear with slope $\mu$, called the aging parameter. In addition, $\mu$ may or may not be a function of temperature in the temperature range of interest for the use of the material.

His effective-time theory for long-term creep makes the following assumptions. First, all mechanical relaxation times change in the same way. They increase by a horizontal shift factor, $a_{e}$, which he defines as

$$
a\left(t_{e}^{\prime}, t_{e}\right)=\frac{M\left(t_{e}^{\prime}\right)}{M\left(t_{e}\right)}
$$


where $\mathrm{a}_{\mathrm{e}}$ is the horizontal shift factor, $\mathrm{M}$ denotes the segmental mobility, and $\mathrm{t}_{\mathrm{e}}$ is the age of the material.

Second, the acceleration factor, $a_{e}$, varies with the age of the material, $t_{e}$, according to a power law. He theoretically derived and confirmed by experiments that the power laws remain valid and the exponent $\mu$ remains constant for very long periods of time. He defines an effective time interval $\mathrm{d} \lambda$ by

$$
d \lambda=a(t) d t
$$

where $\lambda$ is the effective time and $\mathrm{a}(\mathrm{t})$ is the acceleration factor. This is based on the fact that at creep time $\mathrm{t}$, the shift factor is less than unity. All relaxation processes are $1 / a_{e}$ times slower than at $\mathrm{t}=0$. Based on this, the effective time, $\lambda$, was defined as

$$
\lambda=\frac{t_{e}}{1-\mu}\left[\left(1+\frac{t}{t_{e}}\right)^{1-\mu}-1\right]
$$

where $\lambda$ is the effective time, $\mu$ is the aging parameter, $t_{e}$ is the age of the material, and $t$ is time.

To obtain a momentary creep curve in order to predict the creep behavior of the material Struik proposes that the time-temperature superposition method, TTSP, be used. He discusses the physical meaning of the TTSP curve. He uses the argument that for all polymers the TTSP method works very well, therefore it cannot be considered an arbitrary data reduction method.

Sharma [3] explains that creep is one of the simplest experiments that can be used to describe the viscoelastic behavior of polymers. He further discusses that the retarded elastic deformation is due to the interplay of elastic and viscous effects and is a 
characteristic of polymers. Creep compliance monotonically increases with time as the material moves from the glassy region (perfect elastic behavior). For a linear viscoelastic solid, creep strain can be related to the stress by

$$
\varepsilon_{x y}=J(t) \sigma_{x y}
$$

where $J(t)$ is the torsional compliance, $\varepsilon$ is the strain, and $\sigma$ is stress.

He further explains that the stress is found to decrease with time when a material is subjected to an instantaneous strain $\varepsilon_{\mathrm{xy}}$ and can be expressed by the relation

$$
\sigma_{x y}=G(t) \varepsilon_{x y}
$$

where $G(t)$ is the relaxation modulus function. In stress relaxations the material behaves like an elastic solid at very short times and over time the material shows viscoelastic behavior. Sharma states, "The retardation spectrum, $J(\lambda)$ does not represent the response of the materials in any specific test. The retardation spectrum gives the closest representation of molecular processes occurring in viscoelastic deformation."

Wang, et al. [4] investigated the effect of physical aging on the viscoelastic creep properties of a thermoplastic-toughened cyanate ester resin (Fiberite 954-2) and its IM8/954-2 composites, and a semi-crystalline thermoplastic (Fiberite ITX) and its IM8/ITX composites. These tests were preformed using dynamic mechanical analysis (DMA) and tensile creep tests. Creep tests were conducted up to an aging time of $54 \mathrm{hr}$ with the logarithmic aging shift rate, $\mu$, and its dependence on sub-glass transition temperature, being determined. Momentary creep tests were conducted on both material systems at temperatures between $140{ }^{\circ} \mathrm{C}$ and $200{ }^{\circ} \mathrm{C}$ to study the effect of long-term aging. Master curve plots were drawn from these momentary creep tests using the timetemperature superposition principle (TTSP). Effective time theory (ETT) was then used 
to modify the TTSP and incorporate physical aging effects. Their study concluded that physical aging had a significant effect on the creep behavior of the 954-2 and ITX resinbased composite systems. They determined that as the aging time increased, the creep compliance decreased consistently. ETT was then used to predict the long-term creep behavior of the ITX system with reasonable accuracy. Due to the difficulties in rejuvenation, possible post-curing, degradation, and possible phase separation in the cyanate ester system, ETT could not be applied to Fiberite 954-2. Instead, their experimental results indicated that at longer creep times, $t>26000 \mathrm{~s}$, the creep curve could be modeled using the power law

$$
J_{\beta}(t)=J_{\beta_{o}}+m_{1} t^{n}
$$

where $J_{\beta}(t)$ is the creep compliance in the b region, $J_{\beta_{o}}$ is the initial compliance, $\mathrm{m}_{1}$ is the linear coefficient, $\mathrm{t}$ is time, and $\mathrm{n}$ is the relaxation time. While at shorter creep times, $1<\mathrm{t}<3475 \mathrm{~s}$, the creep compliance is best represented by Struik's relationship for tensile creep compliance

$$
J_{\alpha}(t)=J_{\alpha_{o}} \exp \left(t / t_{o}\right)^{m}
$$

where $J_{\alpha_{o}}$ is the initial compliance, $\mathrm{t}$ is time, and $\mathrm{t}_{\mathrm{o}}$ is a characteristic retardation time, and $\mathrm{m}$ is a shape parameter for each creep curve.

Knox, et al. [5] investigated creep analysis of adhesively bonded connections in glass reinforced epoxy pipes. Their approach concluded that the time-temperature superposition principle and the time-temperature stress superposition principle do not take into account physical aging and they are generally not applicable in the region of practical concern, under the glass transition temperature. They further validate the use of 
Findley's power law [6] to model the creep behavior over a long time span. The basic form of this approach is the power law with non-linear coefficients

$$
\varepsilon(t)=\varepsilon_{o}+m t^{n}
$$

where $\varepsilon(t)$ is the strain at time $t, \varepsilon_{o}$ the time independent strain, $m$ the coefficient of time dependent term and $\mathrm{n}$ is a constant independent of stress. Their data showed that viscoleastic deformation occurs and that creep occurs instantaneously. Their experimental results demonstrated that the creep rates have a definite dependency on temperature and stress.

Bradshaw and Brinson [7] present an analytic method based on error minimization, for the data reduction of the study of physical aging and for time-aging time superposition. Their analytic method used error minimization to determine the momentary curves for a set of test data, and to find the associated shift factors and master curve. Their computer program uses the Kohlrausch model

$$
S\left(t ; S_{o}, \tau, \beta ; t_{e}\right)=S_{o} \exp (t / \tau)^{\beta}
$$

presented in Struik to model their data, where $S_{o}$ is the initial compliance, $t$ is time, and $\tau$ is a characteristic retardation time, and $\beta$ is a shape parameter for each creep curve. When shifting data to the momentary curve to obtain a master curve, they select the longest aging time curve as the reference curve, since it spans the longest test time range. They further incorporate a total reference curve, which uses all of the short-term compliance curves to determine the parameters. They felt that the information about the shape of each curve is not fully represented by choosing one curve as a reference curve. Our experience with their computer program was disappointing. 
Beckmann et al. [8] performed creep experiments in uniaxial extension to explore the kinetics of the physical aging process in semicrystalline syndiotactic polystyrene (sPS) having two processing histories. Their test revealed that classical time-aging time superposition, TAS, behavior was found for both materials at temperatures from 70 to $95^{\circ} \mathrm{C}$ with the shift rate, $\mu$, decreasing as temperature was increased. They represented the creep behavior at each time using a Kohlarush-Williams-Watts (KWW) function

$$
D(t)=D_{o} \exp \left(t / \tau_{o}\right)^{\beta}
$$

where $\tau_{\mathrm{o}}$ is a characteristic retardation time and $\beta$ a shape parameter for each creep curve, and $\mathrm{D}_{\mathrm{o}}$ is a fitting parameter. They found the time shift factor $\mathrm{a}_{\text {te }}$ using the methods described by Struik. The mechanical tests were carried out in uniaxial extension in creep conditions. The load was controlled using a computer-controlled servo-hydraulic testing machine, equipped with an oven for temperature control. Sample strain was measured with an extensometer attached to the sample. Their aging experiments were carried out by first annealing the specimens at $160{ }^{\circ} \mathrm{C}$ for $1 \mathrm{hr}$. They then placed the samples in the test machine at the aging temperature, and began applying the "probe" stresses. Experiments were performed at aging test temperatures of $60,70,80,82.5,85,90,95$, 100,110 , and $120{ }^{\circ} \mathrm{C}$ following Struik's protocol for the loading history.

They concluded that if the shape of the creep curve is unaltered as the aging time progresses, that TAS can apply. Thus, the TAS is not limited to the KWW type of creep function and can be represented by any function that describes the data. Their experimental observations suggest that the sPS behaves much like an amorphous polymer below $T_{g}$, while above $T_{g}$ the response is unlike either amorphous materials or other semicrystalline polymers. 
Sullivan [9] demonstrated that physical aging is an important factor in the viscoelastic behavior of composites. He looked at the short-term creep behavior of a unidirectional composite material and of its corresponding resin. Next, he used timeaging time superposition to form momentary creep master curves and discussed the effect of temperature on the physical aging process. The author discusses the appropriateness of TTSP for use in making both momentary and long term creep master curves in polymer composites. The effective-time theory of Struik is used in the prediction of long-term creep behavior.

Sullivan tested a unidirectional glass fiber reinforced thermosetting resin system. It was comprised of 30 vol.\% glass fibers and Dow Derakane 470-36 resin. The $T_{g}$ for this system is around $125-130{ }^{\circ} \mathrm{C}$. All test were conducted in tension using tensile strips of dimensions $150 \mathrm{~mm} \times 13 \mathrm{~mm} \times 3 \mathrm{~mm}$. Both short term (momentary) and long term creep tests were conducted. For the short-term test Sullivan followed Struik's snap shot assumption and in long term creep tests, aging occurs simultaneously with the creep. Annealing and rejuvenation of test specimens was done at $170^{\circ} \mathrm{C}$ for $2 \mathrm{hrs}$.

Sullivan concluded that the creep compliance curves are dependent on the aging time, much like the results reported for glassy polymers. Shifting the momentary compliance curves outlined by Struik formed the momentary master curves. Using both horizontal and vertical shifting, his data showed excellent superposition.

Chai and McCrum [10] studied the mechanism of physical aging in crystalline polymers by thermally stimulated creep and by creep superposition. Their goal was to model an experiment where the specimen was heated to a temperature, $\mathrm{T} 2$, then quenched to and maintained at T1. The specimen's viscoelastic parameters then changed with time 
due to aging. They observed that aging for an elapsed time had no effect on retardation elements at $\tau$ for $\tau>>t_{\mathrm{e}}$.

McCrum [11] describes the computer modeling of physical aging using the Sequential Aging Theory. Chai and McCrum did not support the Sequential Aging Theory with numerical modeling, but this paper proposes a numerical application of the theory. He noticed considerable changes if the specimen is quenched to liquid nitrogen temperature and data was taken on the run as the temperature increased. Quenching increases the magnitudes of the $\beta$ - and $\gamma$-relaxations and if there is a shift of viscoelastic spectra produced by quenching, it isn't large.

Menard [12] characterizes the $\gamma, \beta$, and $\alpha$ relaxations in the following way:

The gamma transition is where the material begins to heat up and expand. Here, bending and stretching and side chain movements can occur due to an increase in the free volume. The beta transition is where the material begins to develop toughness. This occurs as the material continues to heat up and the whole side chains and localized groups of four to eight backbone atoms start to have enough space to move. The alpha transition is a slippage of the crystallites past each other.

McCrum further explains that small shifts of the spectrum in polymeric solids due to quenching and aging are conceivable. Sequential aging begins after a quench to temperature $\mathrm{T}$ where the viscoelastic elements with very small retardation times are aging. The elements are moving to their appropriate values corresponding to the temperature. The sequential aging theory was proposed to explain two experimental facts: 
- The pronounced change in shape of the retardation spectrum produced by aging, first observed by Kovacs et al. [13] and others [14], [10].

- Observations from thermally stimulated creep experiments in which, during aging up to a time $t_{\mathrm{e}}$, the retardation times at $\tau$ greatly in excess of $t_{e}$ do not age [10].

McCrum concludes that physical aging is not restricted to the temperature region between the alpha- and beta-relaxations, as originally proposed by Struik.

Guerdoux et al. [15] studied the physical aging properties of polycarbonate and poly (methyl methacrylate). They measured the aging properties by dynamic mechanical experiments at different temperatures. They argue that the horizontal shift proposed by Struik to superpose the experimental curves for different aging times is only a first approximation. They state, "Struik proposed that the primary mechanism of physical aging arises from changes in molecular mobility of the polymer segments. This results in a proportionate increase in all retardation times, which can be represented by a shift with aging time of the retardation time spectra towards longer times. Thus, creep curves in the linear viscoelastic region can be superposed by a 'nearly horizontal' shift along the $\log$ (time) axis." The aim of their study was to see if Struik's proposal could be generalized from creep to embrace stress relaxation and dynamic mechanical experiments. They measured the torsional viscoelastic properties of polymers with a Rheometrics mechanical spectrometer. They tested specimens with both cylindrical and rectangular cross-sections. The effective length of the cylindrical specimens was $40 \mathrm{~mm}$ and their diameter was $5 \mathrm{~mm}$. The parallelepipeds had the following dimensions: length 
$40 \mathrm{~mm}$, width $11 \mathrm{~mm}$, thickness $4 \mathrm{~mm}$. The specimens were annealed for one hour at 20 ${ }^{\circ} \mathrm{C}$ above their $\mathrm{T}_{\mathrm{g}}$. The specimens were then quenched to near ambient conditions.

Their testing procedure followed the snapshot assumption of Struik. They measured the relaxation modulus, $G^{\prime}(\omega)$ and the dynamic modulus, $G^{\prime \prime}(\omega)$. They tried to superimpose the curves using only horizontal shifts and obtained different values of $a_{v}$ for the three types of measurements. They concluded that the simple hypothesis of Struik is not valid for these data. They then used the model proposed by Chai and McCrum. In this model, it is assumed that the limiting values of the viscoelastic function vary with aging time. This hypothesis gives directly by a simple calculation the three following equations

$$
\begin{gathered}
G^{t_{v_{o}}}(t)=C_{v} G^{t_{v}}\left(t a_{v}\right)+B_{v} \\
G^{\prime t_{v_{o}}}(\omega)=C_{v} G^{\prime t_{v}}\left(\omega / a_{v}\right)+B_{v} \\
G^{\prime \prime t_{v o}}(\omega)=C_{v} G^{\prime \prime t_{v}}\left(\omega / a_{v}\right)
\end{gathered}
$$

where $t_{v_{0}}$ is the reference aging time, $B_{v}$ and $C_{v}$ describe changes in the relaxed and unrelaxed moduli. These equations imply that to superimpose the experimental curves it is necessary to make a vertical shift and a rotation, as well as the horizontal shift $\mathrm{a}_{\mathrm{v}}$. They conclude that the horizontal shift proposed by Struik to superimpose $G(t), G^{\prime}(\omega)$ of $\mathrm{G}^{\prime \prime}(\omega)$ for different aging times is only a first approximation and is not valid for the entire range of time or frequency.

Dean et al. [16] studied the creep behavior for poly(butylene terephtalate, PBT) over a range of elapsed times, $t_{e}$, between quenching from $80{ }^{\circ} \mathrm{C}$ and the start of the creep test. They tested specimens of PBT (POCAN B1505) that were $145 \mathrm{~mm} \times 12 \mathrm{~mm}$ x $6 \mathrm{~mm}$ for creep tests and $145 \mathrm{~mm} \times 10 \mathrm{~mm} \times 3 \mathrm{~mm}$ for dynamic tests. They then heated the 
specimens to $80{ }^{\circ} \mathrm{C}$ for $30 \mathrm{~min}$, quenched them in water at room temperature then stored them at $23{ }^{\circ} \mathrm{C}$ for various periods of time. They used the following equation to model the compliance in the $\alpha$ region

$$
D_{\alpha}(t)=\left(D_{R_{\alpha}}-D_{U_{\alpha}}\right)\left[1-\exp -\left(t / \tau_{\alpha}\right)^{m}\right]
$$

where $m$ is a parameter characterizing the width of the retardation time spectrum of the mechanism and $D_{U_{\alpha}}$ and $D_{R_{\alpha}}$ are compliances for the $\alpha$ process. They then expanded the exponential term in this function as a power series which gives

$$
D_{\alpha}(t)=\Delta D_{\alpha}\left[\left(t / \tau_{\alpha}\right)^{m}\left(1-\frac{1}{2}\left(t / \tau_{\alpha}\right)^{m}+\ldots\right)\right]
$$

Their results indicated a small variation in $m$ with elapsed time. Analyses in which $m$ was considered to vary with $t_{\mathrm{e}}$ were significantly more complicated than when $m$ was constant. They then used a constant, average value of $m$ to fit the data.

\subsection{Objectives}

The purpose of this study was to develop a low-cost, short-term testing procedure to predict the time dependent properties of commercial PVCs and High Density Polyethylene over the lifetime of the installation (50 years). Bending tests, in accordance with ASTM D6272-98 were used to produce momentary curves of creep compliance at various aging times, $t_{e}$, and temperatures $T_{i}$. Aging times were chosen following Struik's snapshot assumption. Data taken at the different temperatures (TTSP) and the effectivetime theory (ETT) were then used to develop a model predicting the time-dependent properties of the liner. 


\subsection{Organization}

This thesis is organized into six chapters. The first presents the scope of the project, a literature review of relevant work, and the project's objectives. Chapter two discusses the materials tested and the process in which they were tested. The research itself was divided into parts. Chapter three presents the testing method and data reduction of the effective-time theory. The testing method and data reduction for the time temperature superposition principle is discussed in chapter four. Chapter five compares the data from long-term testing at different temperatures and compares the data with full-scale tests. Lastly, chapter six presents a summary of the findings and recommendations. 

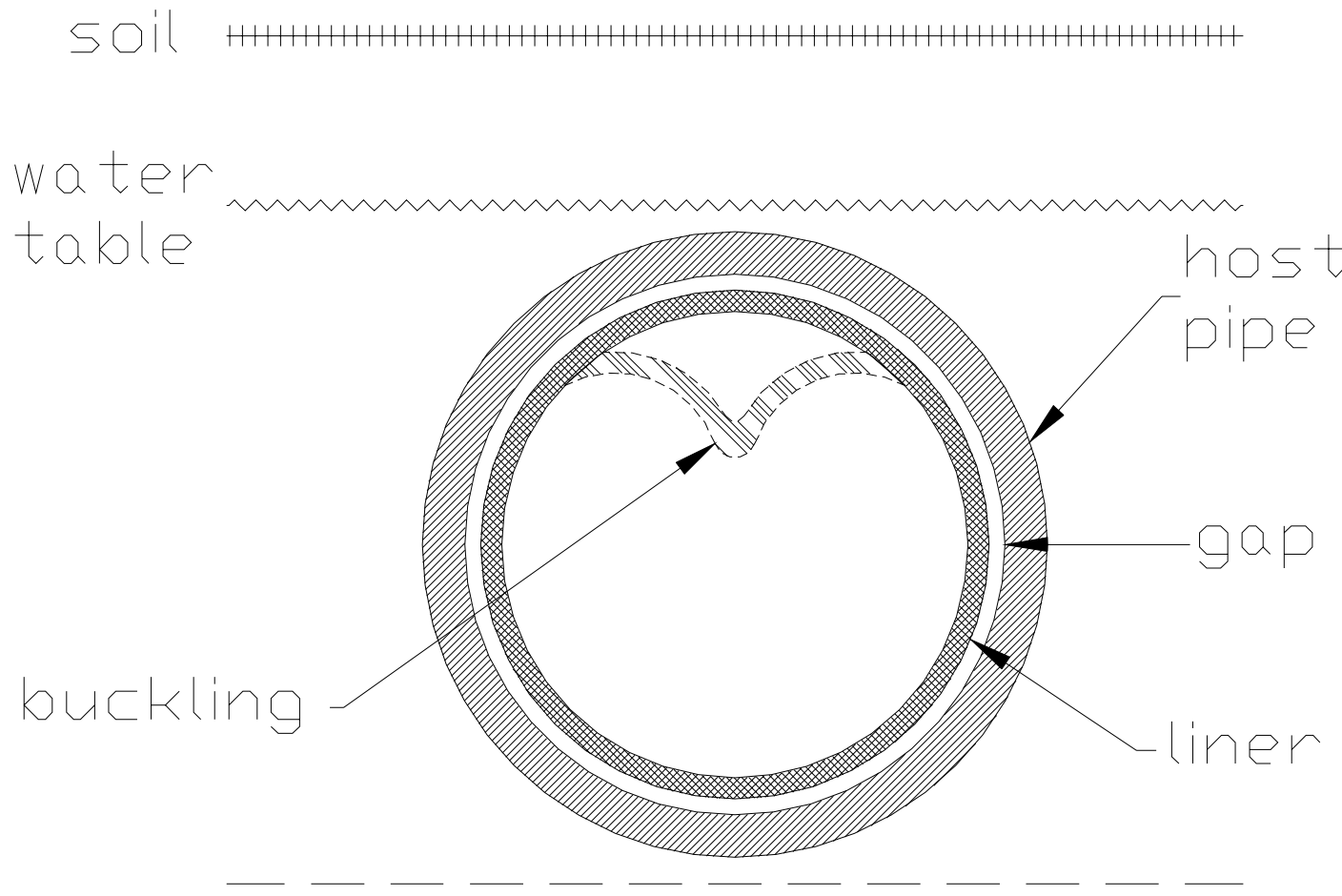

Figure 1.1. Illustration of trenchless lining of a pipe.

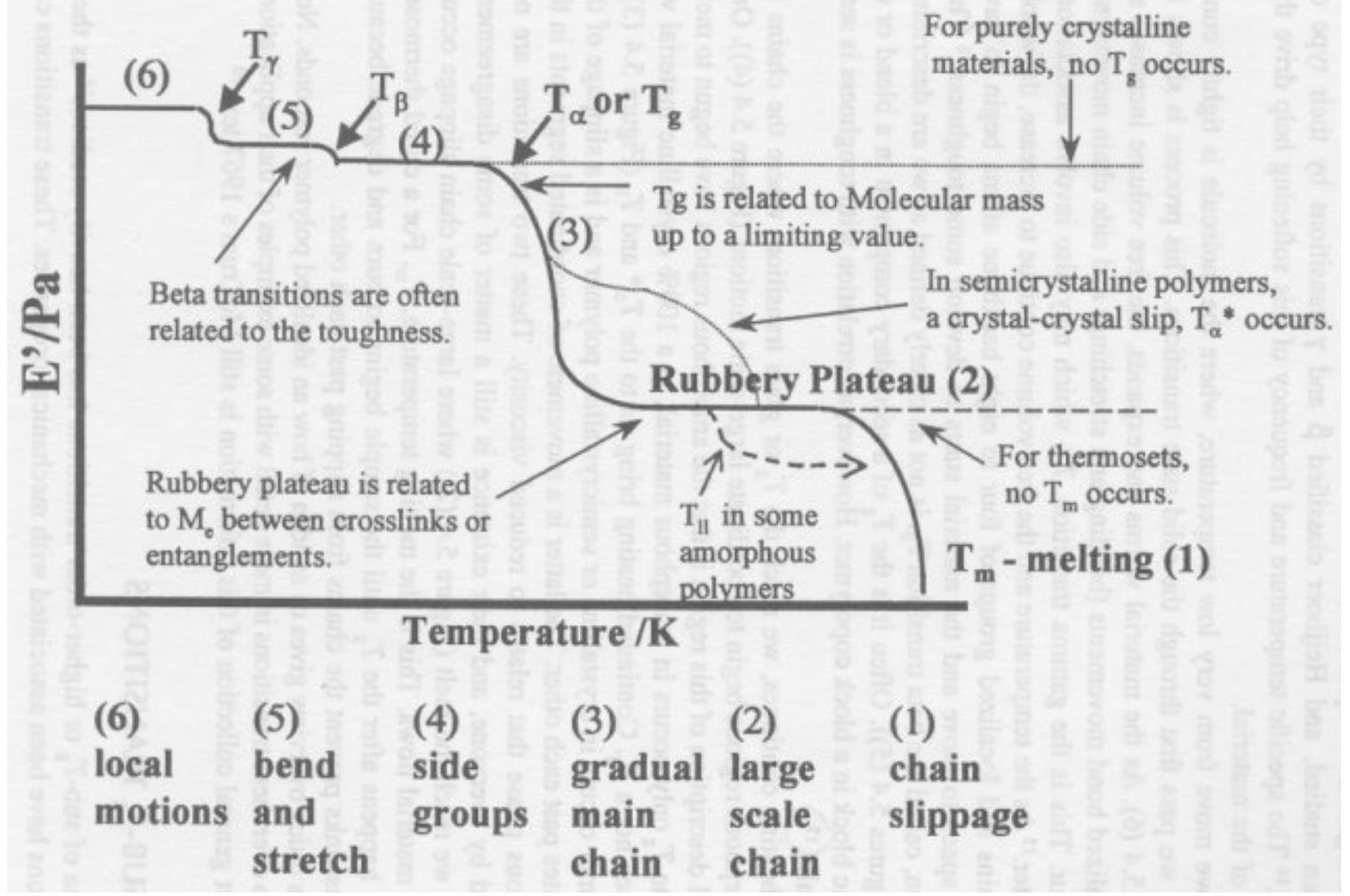

Figure 1.2. Idealized temperature scan of a polymer [Fig. 5.4, 12]. 


\section{CHAPTER 2 EXPERIMENTAL PROCEDURES}

\subsection{Introduction}

The relationship between stress and strain depends on time due to creep [1] and physical aging [2]. In a related study [17], creep and aging were studied in the frequency domain using Dynamic Mechanical Analysis (DMA). However, two drawbacks of Dynamic Mechanical Analysis (DMA) testing motivate long-term bending (LTB): specimen $^{1}$ size and test duration. DMA specimens are small on the order of $(10 \mathrm{~mm} \mathrm{x}$ $1.5 \mathrm{~mm} \times 15 \mathrm{~mm}$ ) and they must be cut from the pipe wall. Instead, LTB specimens use the full thickness of the pipe wall with a maximum testing size of (50 $\mathrm{mm} \times$ material thickness $\times 231 \mathrm{~mm}$ ). The larger size of the LTB specimens allows for a better representation of the material properties to be tested. Running long-term tests on a DMA ties up the machine and is costly. Therefore, another, less expensive method to test specimens needed to be developed. The LTB fixture was designed to perform inexpensive, long-term creep testing under ASTM D6272-98 four-point bending conditions.

\subsection{Experimental Approach}

\subsubsection{Testing Method}

All tests were performed using a four-point loading system, which uses two loading points with a spacing equal to one third of the support span. Testing was done in accordance to ASTM D6272-98.

\subsubsection{Design and Fabrication of Testing Apparatus}

Tests were conducted using the West Virginia University Long-Term Bending Fixture (LTB) based on ASTM D6272-98. This fixture uses two loading points with a 
spacing of $64 \mathrm{~mm}$ (2.52 in) or one third of the support span spacing of $192 \mathrm{~mm}$ (7.56 in).

Four fixtures can support a specimen with a maximum width of $50 \mathrm{~mm}$ (1.96 in) while four other fixtures can support a specimen with a maximum width of $25 \mathrm{~mm}$ (0.98 in). All four-load points have a radius of $7.94 \mathrm{~mm}(0.313 \mathrm{in})$, which allow for a minimum specimen depth of $4.96 \mathrm{~mm}$ (0.195 in) according to ASTM D6272-98 Section 6.2. Loadpoint displacement is measured with a linear optical encoder with an accuracy of 0.005 $\mathrm{mm}$ and a maximum travel of $50 \mathrm{~mm}$. The optical encoder, model LDK-4-4-B and spar and scale assembly, model B36679-4-0-50.0 were purchased from Dynamics Research Corporation. The majority of the testing apparatus was constructed out of aluminum, except for the load supports and the vertical rods where stainless steel was used. Linear bearings were used to allow the carriage to descend freely and apply the load to the specimen (refer to Appendix A for fixture schematics). A picture of the fixture with an untested specimen is shown in Fig. 2.2. Fig. 2.3 is a picture of the testing fixture with a tested specimen. Six fixtures shown during testing are in Fig. 2.4. Each specimen is fixed to the load points with rubber bands, as shown in Fig. 2.5. This allows the top of the specimen to remain in contact with the bottom of the loading arms at all times during the test. Refer to Fig. 2.6 for a depiction of four point bending. Further, this allows an accurate measuring of the deflection of the specimen, since the specimen may not always be flat and enables the fixture to measure the relaxation.

\subsubsection{Data Acquisition}

Data was acquired from the testing fixtures using an ACS-Tech80 5312B ISA, Encoder Interfacing card with four axes. A Pentium 166mhz and Pentium 150mhz computer were used to read the card and store the data (Fig. 2.7). Software drivers 
provided by the DAQ manufacturer were modified to write data to files in specific time increments using Visual Basic 6.0. Refer to Appendix B for the modified Visual Basic code.

\subsection{Test Environmental Conditions}

All tests were preformed in West Virginia University's environmental chamber located in room G86 of the Engineering Science Building. The environmental chamber is a Cincinnati Sub-Zero model Z-32 (Fig. 2.8) that uses a Chromalox 2030 microprocessor controller.

\subsection{Sample Preparation}

\subsubsection{Materials}

In this study five different materials were tested, three of which are PVCs, one is High Density Polyethylene, and the other is Polyester with reinforced felt. The glass transition temperature and melting temperature of the materials were obtained from testing by Dynamic Mechanical Analysis at West Virginia University [17] (Table 2.1). These materials are commercially available, to avoid bias, the materials were designated with the letters $\mathrm{A}, \mathrm{B}, \mathrm{C}, \mathrm{D}$, and $\mathrm{E}$.

\subsubsection{Sample Collection}

New liner samples were produced at the vendor facility or field installation of each material. In either case, the samples were taken from rounded pipe and processed into a cylindrical configuration (Fig. 2.1). All liner samples were cut perpendicular to the direction of extrusion and had a minimum length of $3 \mathrm{ft}$. If the installation prohibited the removal of samples at this length, three samples not less than $1 \mathrm{ft}$ in length each were 
provided. The $3 \mathrm{ft}$ samples collected from the field were cut into three pieces, each with a length of $1 \mathrm{ft}$.

For the purpose of measuring the thickness of the bagging material used on material E, the vendor also provided two samples at least $930 \mathrm{~cm}^{2}$ of un-impregnated hose comprising both main hose and the lining hose. Due to the nature of the thermoset liners, the liner test section had at least one flat surface.

\subsubsection{Flattening of Samples}

Next, the samples, except for material D, were flattened. First, the pipe section was cut longitudinally into three equal sections. These sections were then placed, concave side down, on a piece of flat aluminum with a thickness of $12.7 \mathrm{~cm}(1 / 2 \mathrm{in})$ and overall dimensions slightly greater than the sample and placed in an oven. The orientation of the

sample indicated the hoop-wise direction of the sample as shown in Fig. 2.9. A second identical aluminum plate was placed on top of the sample and additional weights were added to it. The samples, aluminum plates and additional weights were then heated to $\mathrm{T}_{\mathrm{H}}$ and maintained at that temperature for not less than 48 hours. The specimen heating temperature, $\mathrm{T}_{\mathrm{H}}$, was determined to be $15^{\circ} \mathrm{C}$ above the glass transition temperature $\left(\mathrm{T}_{\mathrm{g}}\right)$ of the material, but below it's melting point $\left(\mathrm{T}_{\mathrm{m}}\right)$.

\subsubsection{Sample Sizing and Preparation}

The specimens had a minimum aspect ratio of 16 to 1 and had a maximum width of $50 \mathrm{~mm}$ and a minimum depth of $4.96 \mathrm{~mm}$. All specimens were cut to width using a band saw (Fig 2.10).

The thickness and width of each specimen were measured at both ends and at the center of the span using a micrometer as per Section 6.3 of ASTM D790 and then 
averaged. In the case of the thermoset specimen, the thickness of the bagging material was subtracted from the total thickness. The specimens, except for the HDPE, were annealed in an oven at temperature $\mathrm{T}_{\mathrm{H}}$ for at least $30 \mathrm{~min}$. The HDPE was annealed at the temperature used to expand the liner during installation. They were then quenched between steel plates for approximately $5 \mathrm{~min}$. After the specimens were quenched, they were placed in the fixture and held in place with rubber bands wrapped around the inner loading nodes. Next, the spar and scale assembly was placed in the fixture. Using the software the fixtures were then zeroed, the test filename and path were entered and the data was then recorded. 
Table 2.1. Material Properties.

\begin{tabular}{|c|c|c|c|}
\hline Material & $\mathbf{T}_{\mathrm{g}}\left({ }^{\circ} \mathbf{C}\right)$ & $\mathbf{T}_{\mathbf{H}}\left({ }^{\circ} \mathbf{C}\right)$ & $\mathbf{T}_{\mathbf{M}}\left({ }^{\circ} \mathbf{C}\right)$ \\
\hline$A$ & 70 & 85 & $\sim$ \\
\hline$B$ & 90 & 105 & $\sim$ \\
\hline$C$ & 85 & 100 & $\sim$ \\
\hline$D$ & -30 & 93 & 116 \\
\hline$E$ & 135 & 150 & $\sim$ \\
\hline
\end{tabular}

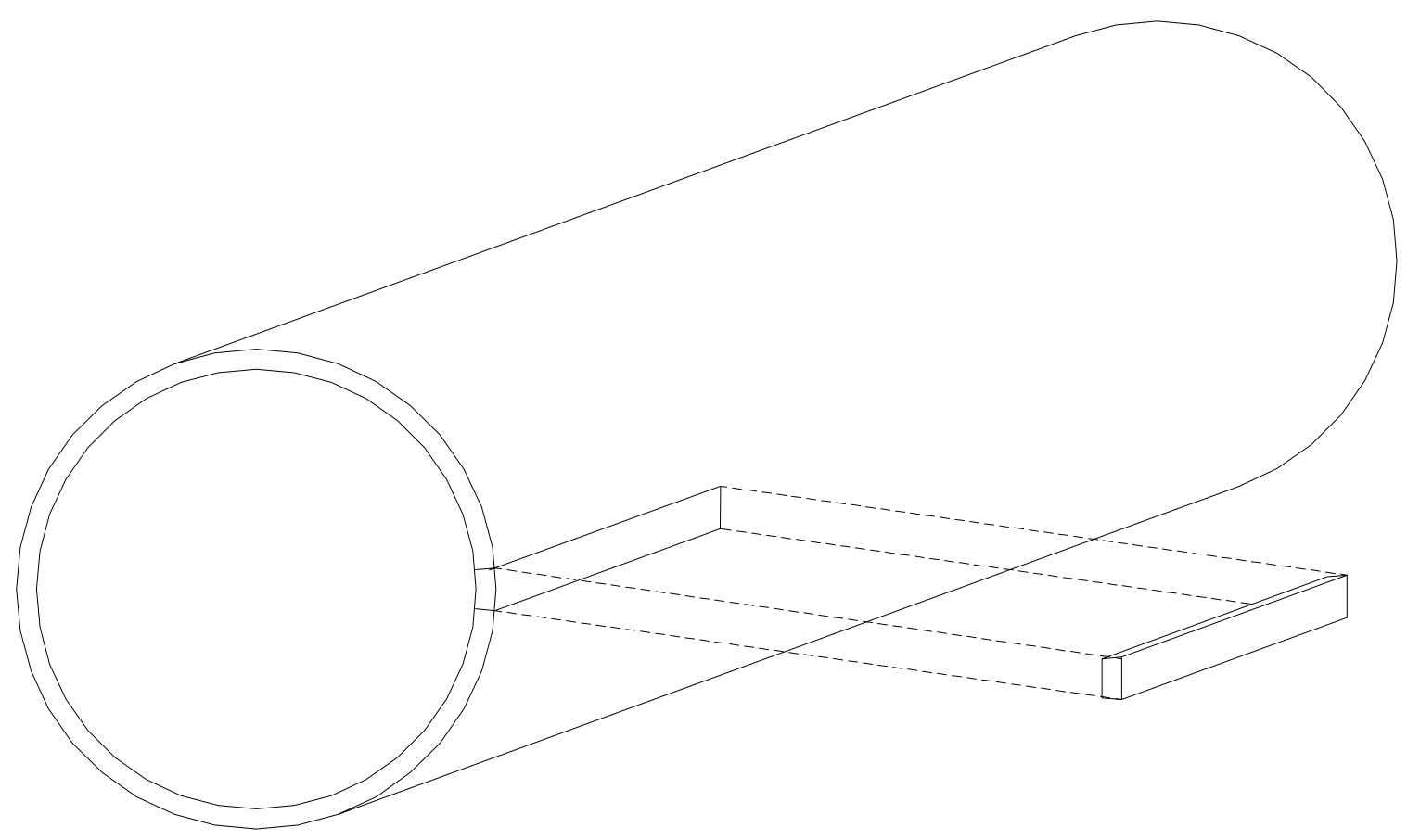

Figure 2.1. Illustration of the location from which the samples are removed. 


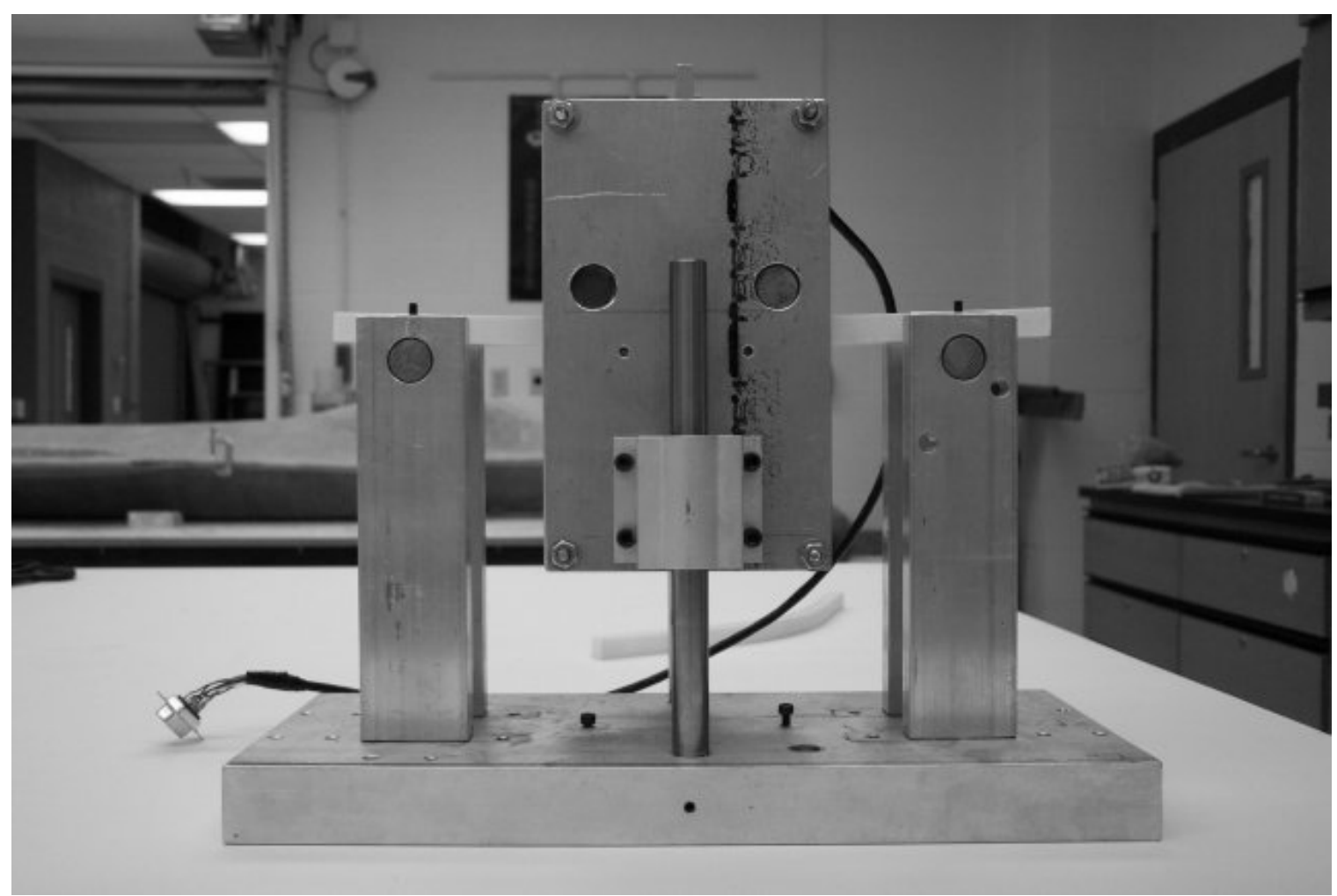

Figure 2.2. LTB fixture with untested specimen.

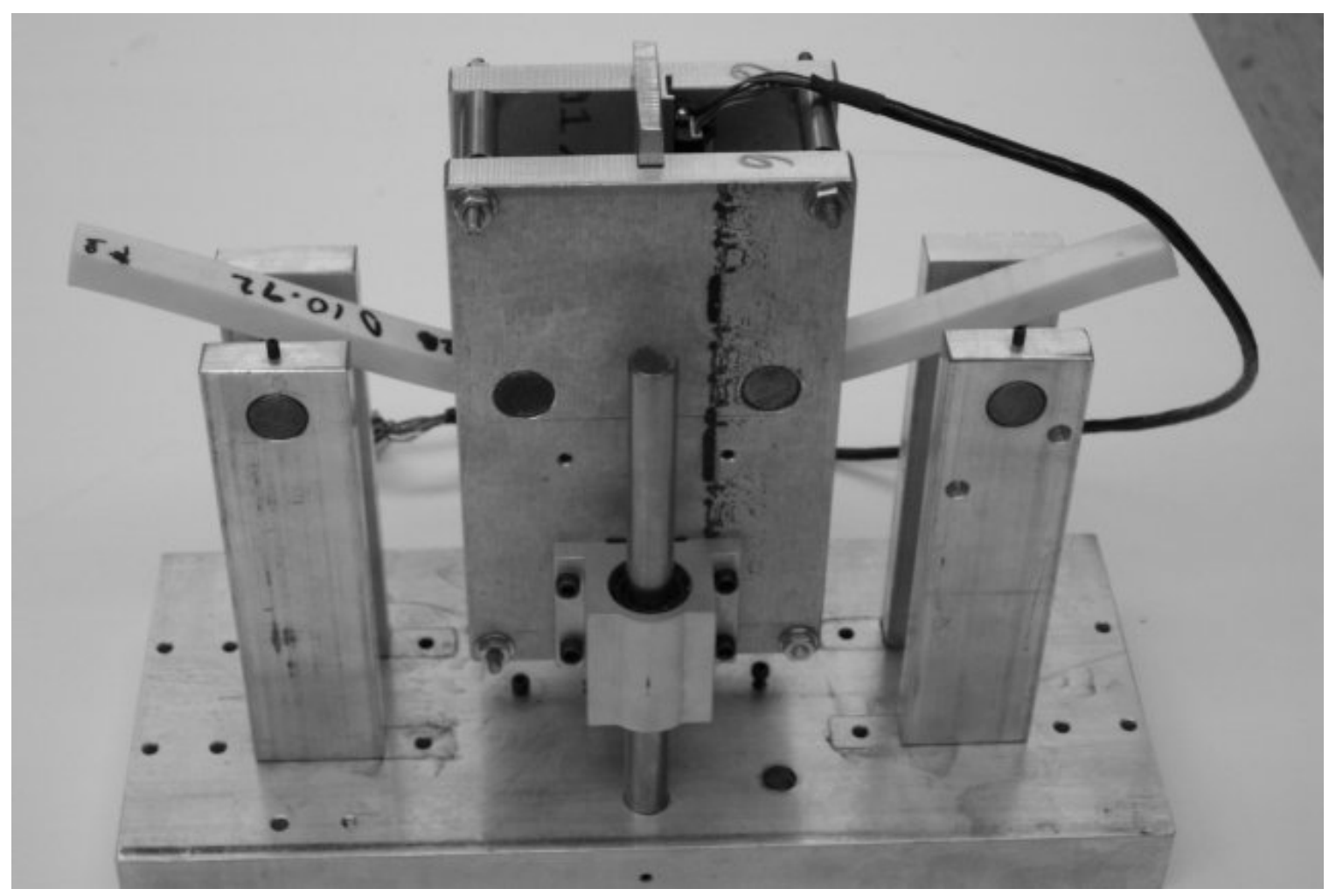

Figure 2.3. LTB fixture with bent specimen. 


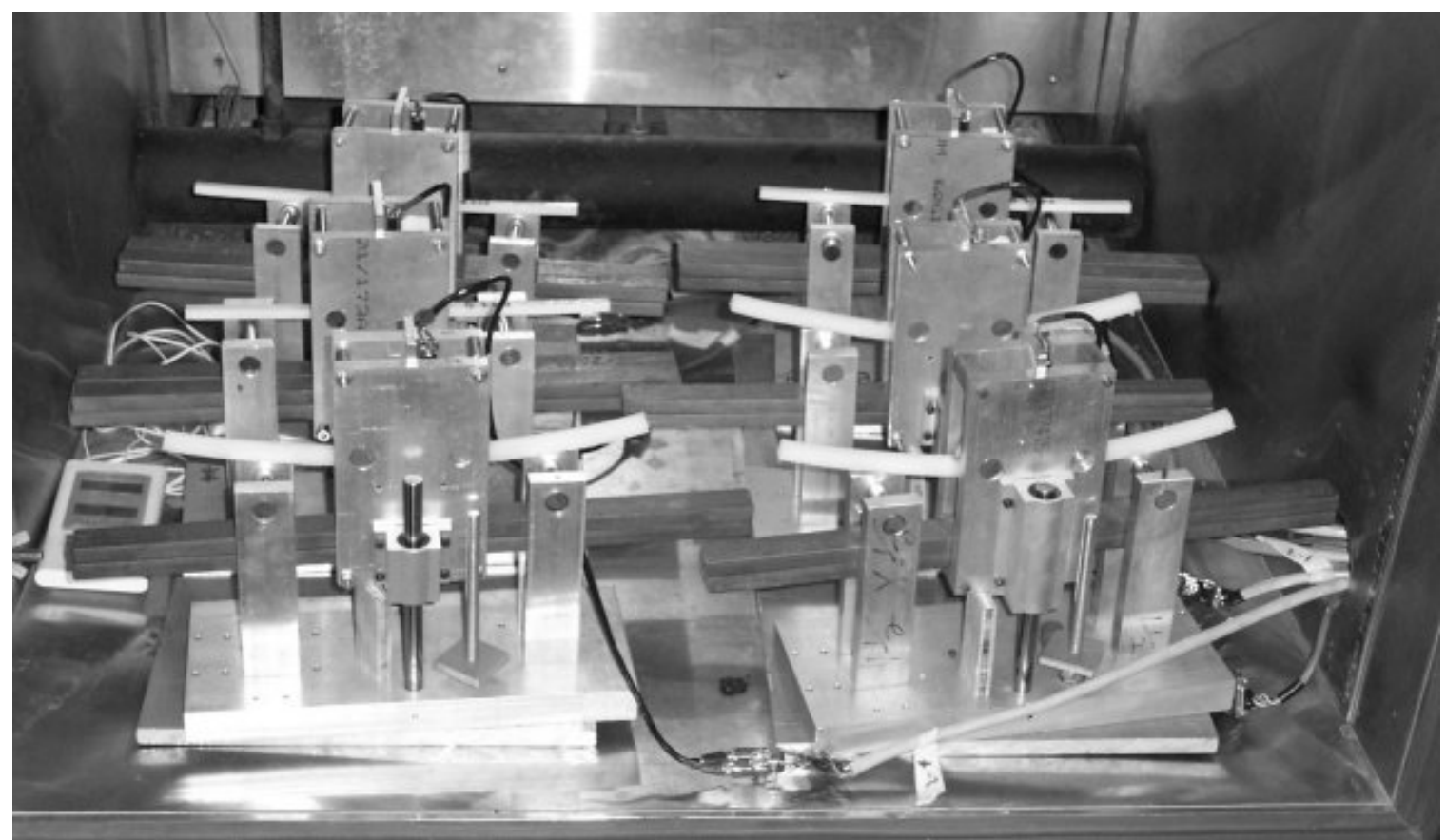

Figure 2.4. LTB fixtures in environmental chamber under testing.

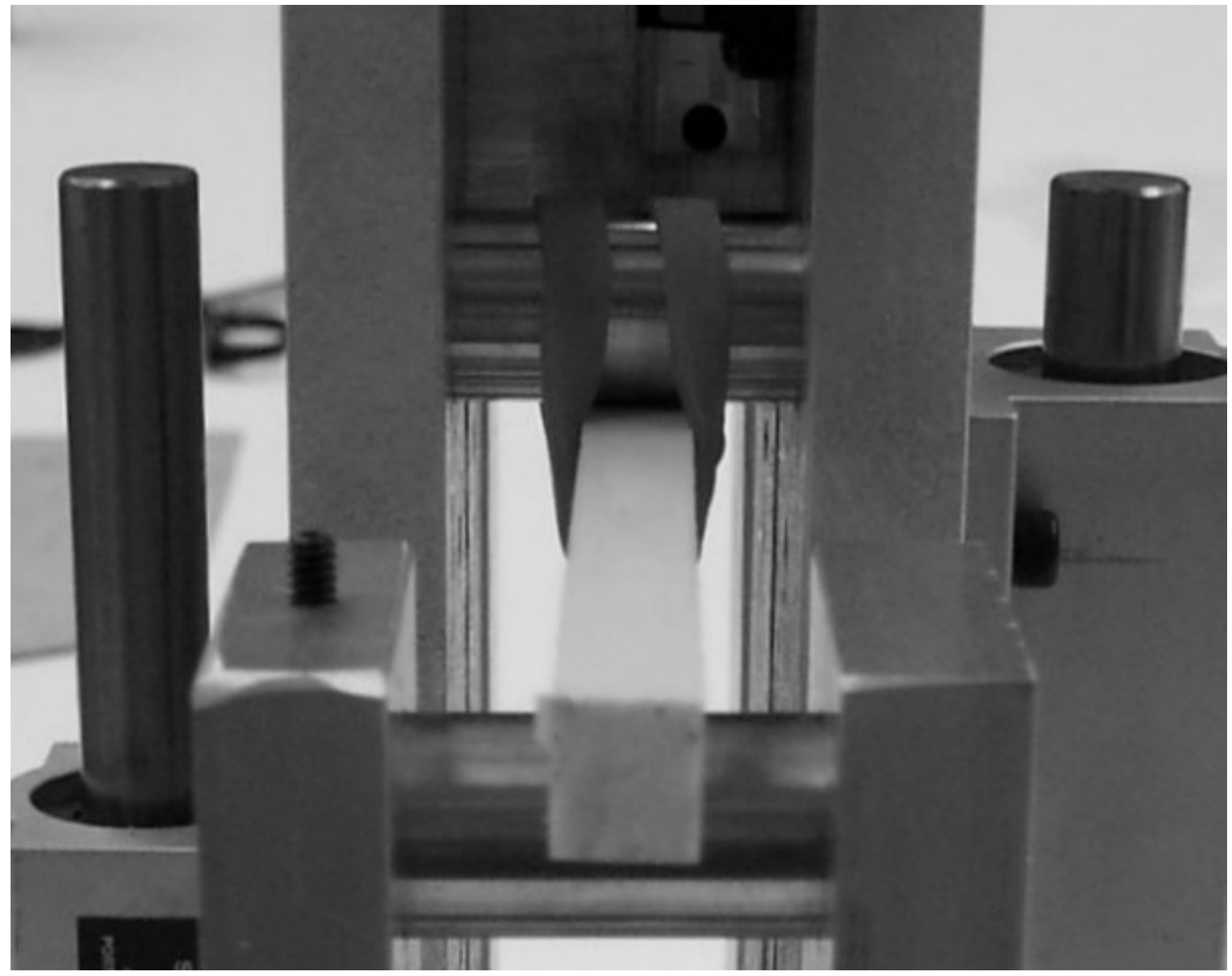

Figure 2.5. Specimen held in place with rubber bands. 


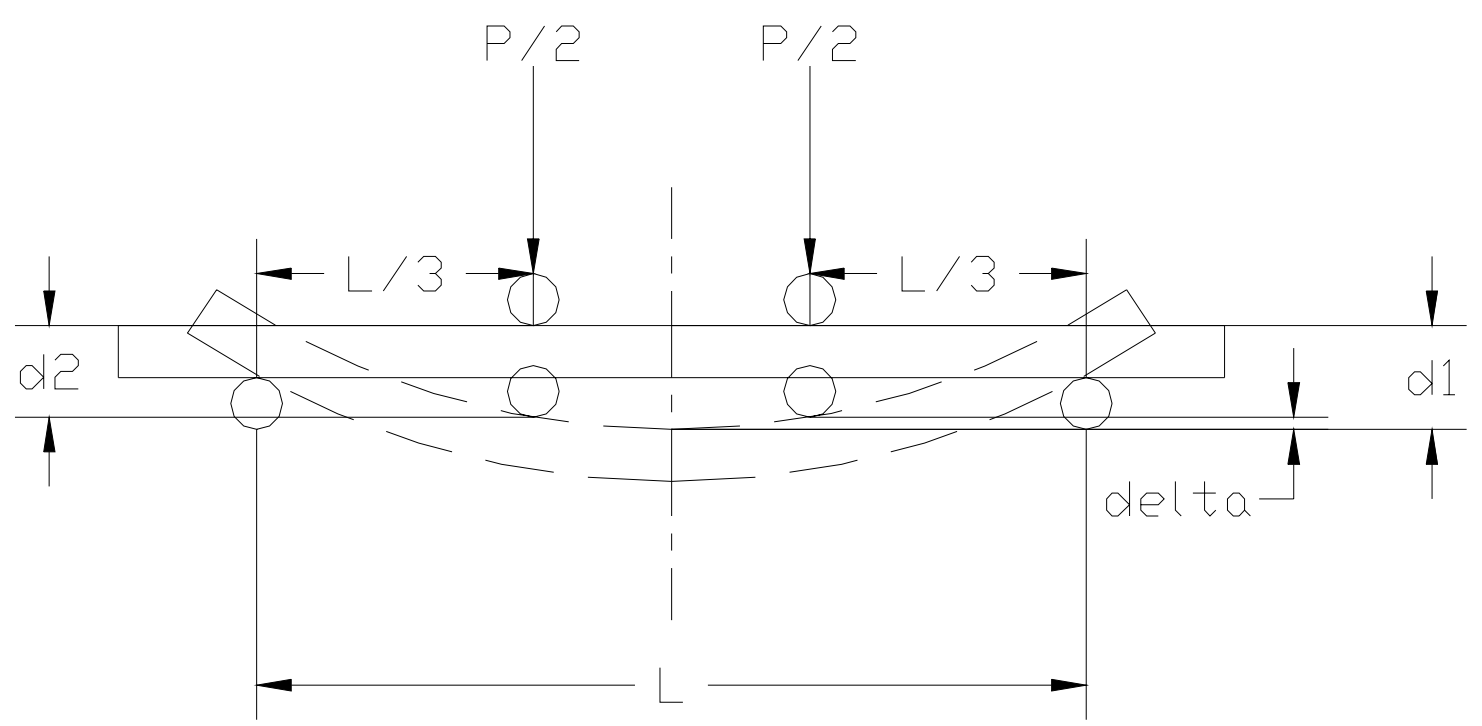

Figure 2.6. Illustration of specimen under four-point bending, the dashed line represents the deformed specimen due to loading.

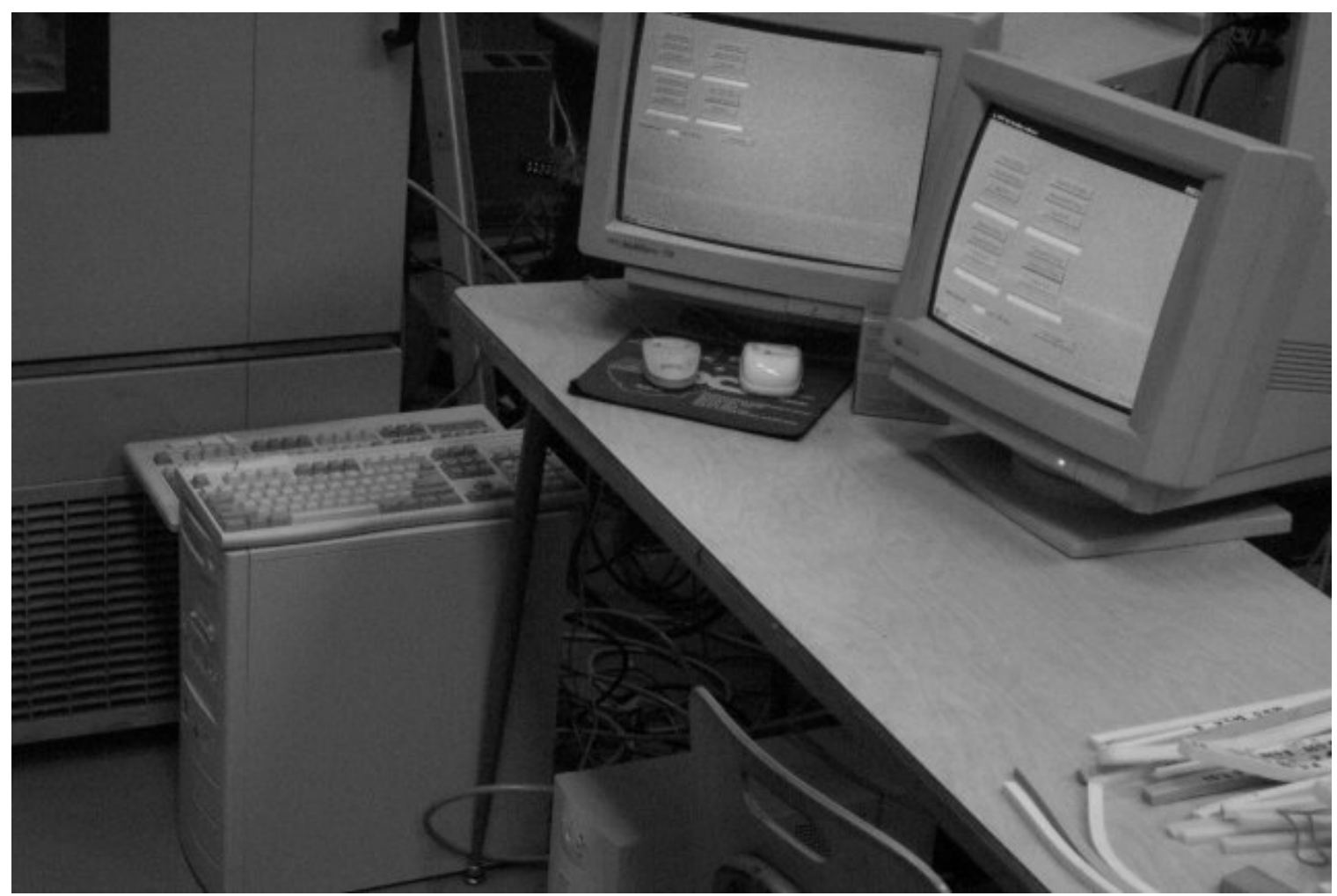

Figure 2.7. Computers used for data acquisition. 


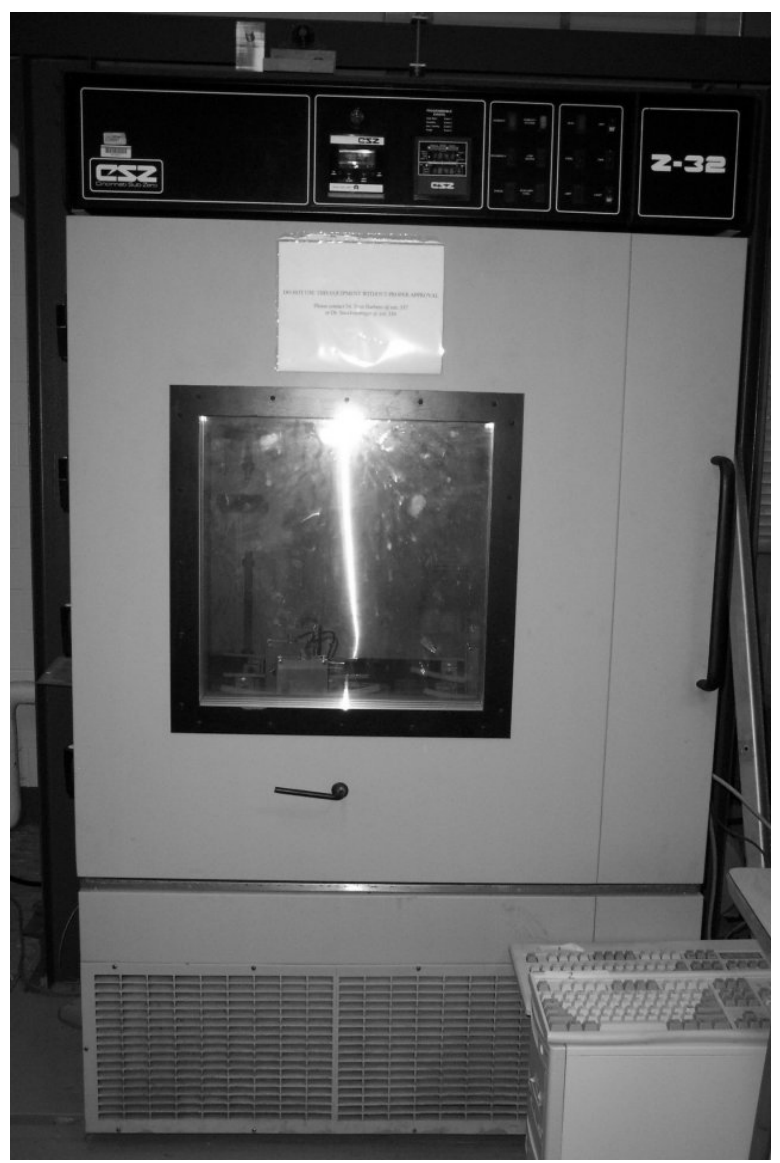

Figure 2.8. Environmental chamber.

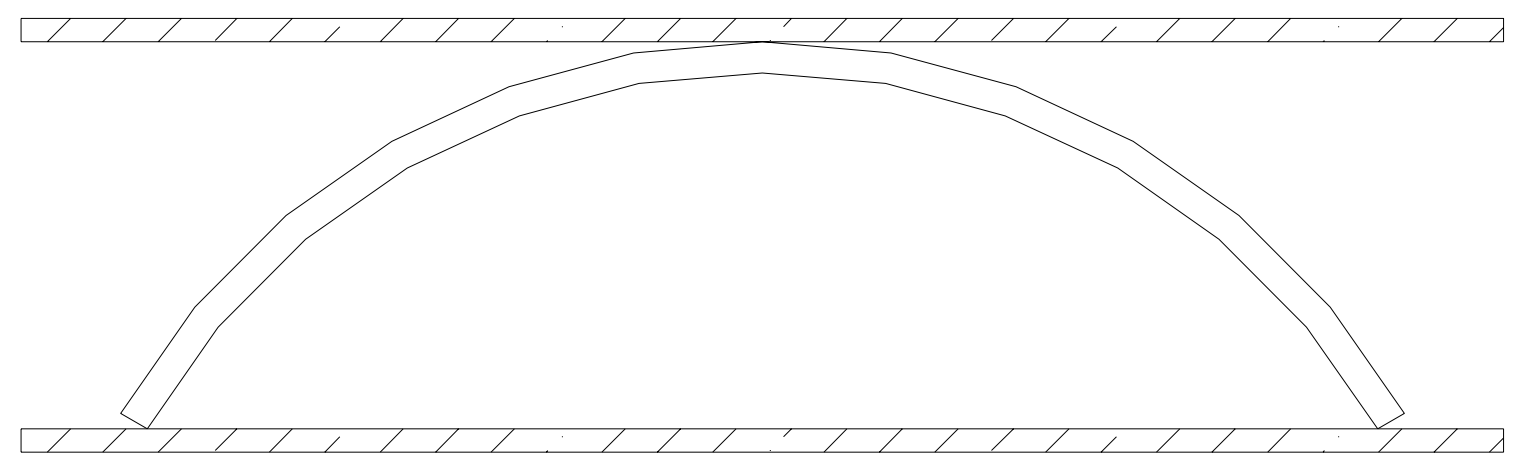

Figure 2.9. Illustration of one-third of pipe between two plates for flattening purposes. 


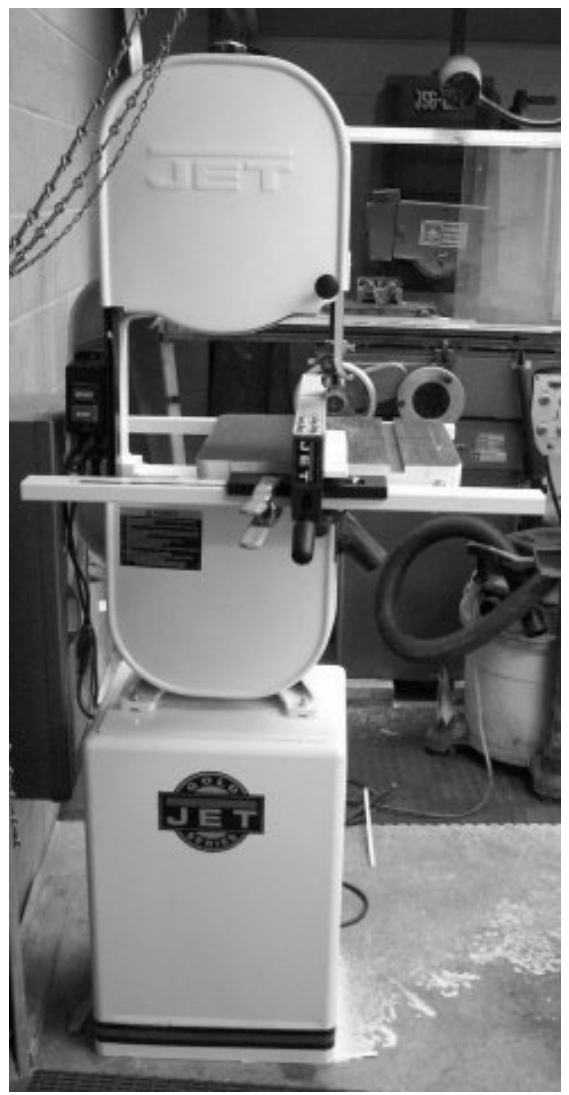

Figure 2.10. Band saw used to cut specimens.

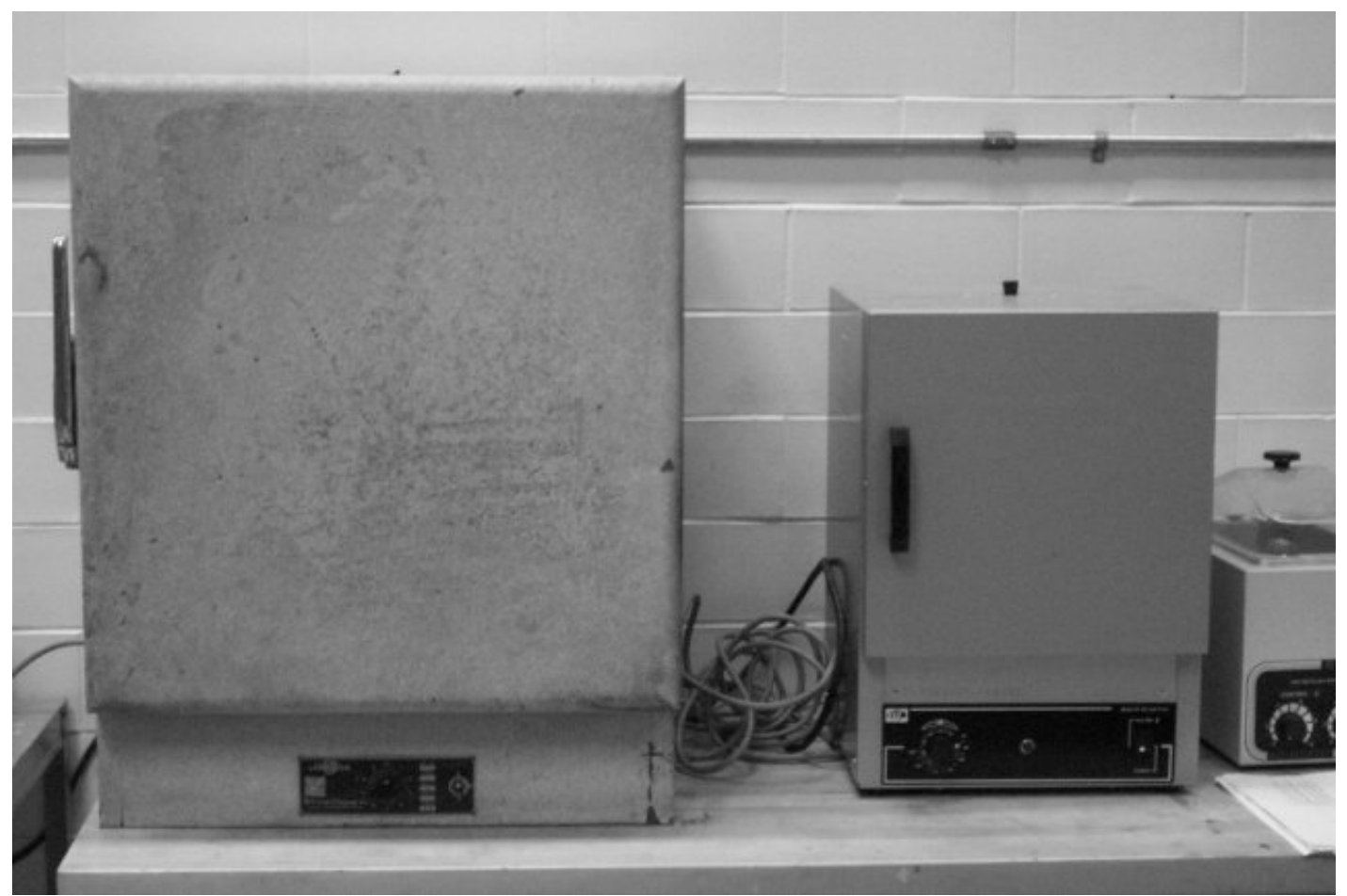

Figure 2.11. Ovens used to anneal specimens. 


\section{CHAPTER 3 AGING STUDY}

\subsection{Introduction}

Physical aging starts when a polymer is quenched to a temperature below its $\mathrm{T}_{\mathrm{g}}$, regardless of whether or not the material is under load. Physical aging is accompanied by increases in stiffness, yield stress, density, and viscosity, and decreases in creep and stress relaxation rates.

The equivalent time theory (ETT) of Struik [2] assumes that physical aging can be explained by a single shift, $a_{e}$, of the entire relaxation spectrum. In a typical Struik-type experiment, the sample is quenched from above the glass-transition temperature, $\mathrm{T}_{\mathrm{g}}$, to the testing temperature, $T_{i}$, and maintained at that temperature for the aging time, $t_{e}$. Tests are then preformed for a short time interval: one-tenth the age, $t_{e}$, of the specimen. This method of testing takes a snapshot of the viscoelastic state, which prevents the data from being tainted by physical aging.

\subsection{Testing Procedure}

Each specimen was prepared as described in Section 2.4.3. Tests were preformed at a temperature, $\mathrm{T}_{\mathrm{i}}$, of $40{ }^{\circ} \mathrm{C}$. Due to room conditions and the capabilities of the environmental chamber the value of $\mathrm{T}_{\mathrm{i}}$ ranged from $39.5{ }^{\circ} \mathrm{C}$ to $40{ }^{\circ} \mathrm{C}$. The width of the specimens was in the range of $7 \mathrm{~mm}$ to $10 \mathrm{~mm}$. The testing temperature and specimen width were determined by doing several practice tests to achieve a deflection large enough that the error in the optical encoder does not interfere with the results. The specimens were tested according to Struik's snapshot assumption. This assumes that if the test time is less than one-tenth the age of the specimen, physical aging is not observed 
during the test time. The testing schedule varied with each study due to other activities, but the snapshot assumption was always followed.

Tests were conducted at eight intervals during the aging experiment. A typical testing schedule is shown in Table 3.1. Conducting an aging study and calculating the shift factors, $a_{e}$, determined the number of test. Using these shift factors, $\mu$ was calculated and tests were preformed until the value of $\mu$ remains constant; that is, the slope of the $\log \left(\mathrm{a}_{\mathrm{e}}\right)$ vs. $\log \left(\mathrm{t}_{\mathrm{e}}\right)$ does not change by adding another data point. For all materials tested, this is accomplished with eight intervals of testing over approximately four days.

\subsection{Data Analysis}

Data reduction was performed using Microsoft Excel 2000 and Microcal Origin 6.0. The files from the Long-Term Bending software were opened in Microsoft Excel. The load was removed after each snapshot test, allowing the sample to relax, but the initial shape is not recovered. This occurs because the relaxation time is finite and because a portion of the viscoelastic behavior is that of a fluid [18]. Each snapshot test starts from a deflected initial position, which is easily found since the optical encoder reads deflection during the relaxation as well as during the creep (snapshot) test. Once the initial deflection was established for each snapshot test, the relative deflection data vs. time represents a snapshot creep curve.

The stiffness of the material increases with age, reducing the amount of deflection observed during the test. Therefore, with each successive test the deflection vs. time curves should lie under one another. This was not observed with the first two tests (refer to Fig. 3.2). This occurrence is thought to be the result of mechanical conditioning, 
where the specimen must adjust and settle onto the load supports. Therefore, the first two tests for each aging study were not used in the calculation of $\mu$.

Next, the compliance $\mathrm{D}(\mathrm{t})$ was calculated for each point. The equation for the compliance under true four-point bending was derived using the method outlined below.

Referring to Figure 2.6, the maximum deflection of a beam under four-point bending with $1 / 3$ load span is [19]

$$
d 1=\frac{-P / 2 b\left(L^{2}-b^{2}\right)^{3 / 2}}{9 \sqrt{3} E I L}+\frac{-P / 2 a\left(L^{2}-a^{2}\right)^{3 / 2}}{9 \sqrt{3} E I L}
$$

where $\mathrm{P}$ is the load, $\mathrm{L}$ is the distance of the support span, $\mathrm{E}$ is the modulus of elasticity, I is the moment of inertia, $a=L / 3$ and $b=L / 3$.

The deflection at the load supports is [19]

$$
d 2=\frac{P / 2^{a^{2} b^{2}}}{3 E I L}+\frac{P / 2 b}{6 E I L}\left[x^{3}-\left(L^{2}-b^{2}\right) x\right]
$$

where $a=L / 3, b=2 L / 3$, and $x=L / 3$. The deflection delta is:

$$
\text { delta }=\delta=d 1-d 2
$$

The moment of inertia of a rectangle is

$$
I=\frac{1}{12} b h^{3}
$$

where $b$ is the width of specimen and $h$ is the height of the specimen.

Thus the compliance is

$$
D=\frac{243(64 \sqrt{6}+135) \delta w h^{3}}{2117 P L^{3}} \approx \frac{33.491 \delta w h^{3}}{P L^{3}}
$$


where $\mathrm{d}$ is the deflection of the specimen, $\mathrm{w}$ is the width of the specimen, $\mathrm{h}$ is the thickness of the specimen, $\mathrm{P}$ is the load, and $\mathrm{L}$ is the distance of the support span.

The next step was to fit a model to the data to obtain an equation for each test. Several models were fit to determine the best fit for the data. These methods included the Standard Linear Solution, Maxwell Model, Dean's Equation [16], and the Modified Power Law. It was determined that a one term Modified Power Law best fit the data. Figure 3.2 is a typical curve fit of the data. The Modified Power Law is represented by the following equation

$$
D=D_{o}+D_{1} t^{m}
$$

where $D_{0}$ is the initial compliance, $D_{1}$ is the linear coefficient, and $t$ is the time, and $\eta$ is the relaxation time. All data fitting parameters were obtained using Microcal Origin 6.0.

Once all of the curves were fit using the Modified Power Law, the next step was to shift the curves to obtain the shift factors and aging value $\mu$ for each material. Shifting the curves turned out to be quite complicated. Dividing each time by a shift factor, $\mathrm{a}_{\mathrm{e}}$, the curves were to be shifted on top of the reference curve. However each of the curves could only be shifted so that a few points would match, creating the problem of which points should be used to align the curves onto the reference curve. To solve this problem it was observed that a straight line in double log scale using the Power Law could represent the data. The Modified Power Law can be transformed into the standard Power Law by the following method

$$
\begin{gathered}
D=D_{o}+D_{1} t^{m} \\
D-D_{o}=D_{1} t^{m} \\
\log \left(D-D_{o}\right)=\log \left(D_{1} t^{m}\right)
\end{gathered}
$$


Finally,

$$
\log \left(D-D_{o}\right)=\log \left(D_{1}\right)+m \log (t)
$$

This approach is similar to Dean et al. Using equation 1.8 and expanding the exponential to only one term, the Modified Power Law can be derived as follows:

$$
\begin{gathered}
D-D_{o}=D_{1}^{\prime}\left[(t / 2)^{m}-\frac{1}{2}(t / 2)^{2 m}+\ldots\right] \\
D-D o \sim D_{1}^{\prime}\left(t / \tau^{m}=\left(D_{1}^{\prime} / \tau^{m}\right) t^{m}\right. \\
D_{1}=\left(D_{1}^{\prime} / \tau^{m}\right) \\
D=D_{o}-D_{1} t^{m}
\end{gathered}
$$

where $m$ is a constant, equal to the average of all the values $m_{i}$ of the aging study, where $\mathrm{i}=1 \ldots \mathrm{N}, \mathrm{N}$ being the number of snapshot test available. The difference between $m_{i}$ and the average $m$ represents a rotation of the line. Chai and McCrum and [10] Guerdoux et al. [15] also discussed the need for rotating the data in addition to horizontal shifting to calculate $\mathrm{a}_{\mathrm{e}}$. Again looking at the Modified Power Law equation (3.6), $\mathrm{m}$ is the slope of the line, therefore averaging this value is the same as performing a rotation of each curve.

Once the data was represented by a straight line, it was noticed that the slopes of each curve did not match. The problem again was which point should be used for the curve shifting. To solve this problem the values of $m_{i}$ (the slope of the curve) were averaged and the data for each snapshot test was refit keeping the value of $m$ constant and equal to the average (Figs 3.4 through 3.8). This produced good results; each curve now was a straight line with the same slope and could be easily shifted on top of one another (Figs. 3.9 through 3.13). Each shift factor was analytically calculated using the following 
method. First, an arbitrary value of $\log \left(D-D_{0}\right)$ was selected. Next, $\log (t)$ was calculated for each test using the following equation

$$
\log (t)_{\text {test }}=\frac{\log \left(D-D_{o}\right)_{\text {arbitrary }}-\log \left(D_{\text {test }}\right)}{m}
$$

where $\mathrm{D}_{\text {test }}$ was obtained from the curve fit of the corresponding test. Then using the last test as a reference, $a_{e}=1, a_{e}$ of each test was calculated using the following formula:

$$
a_{e_{\text {test }}}=\frac{\log (t)_{\text {test }}}{\log (t)_{\text {lasttest }}}
$$

where $\log (\mathrm{t})_{\text {test }}$ is the $\log$ base- 10 of the time of the snapshot curve to me shifted and $\log \left(t^{\text {last test }}\right.$ is the $\log$ base- 10 of the time of the reference snapshot curve. Since $m$ is the same for all snapshot tests, the value of $\log \left(D-D_{0}\right)$ does not affect the results of (3.11). Below is a flowchart describing the process of calculating the shift factors.

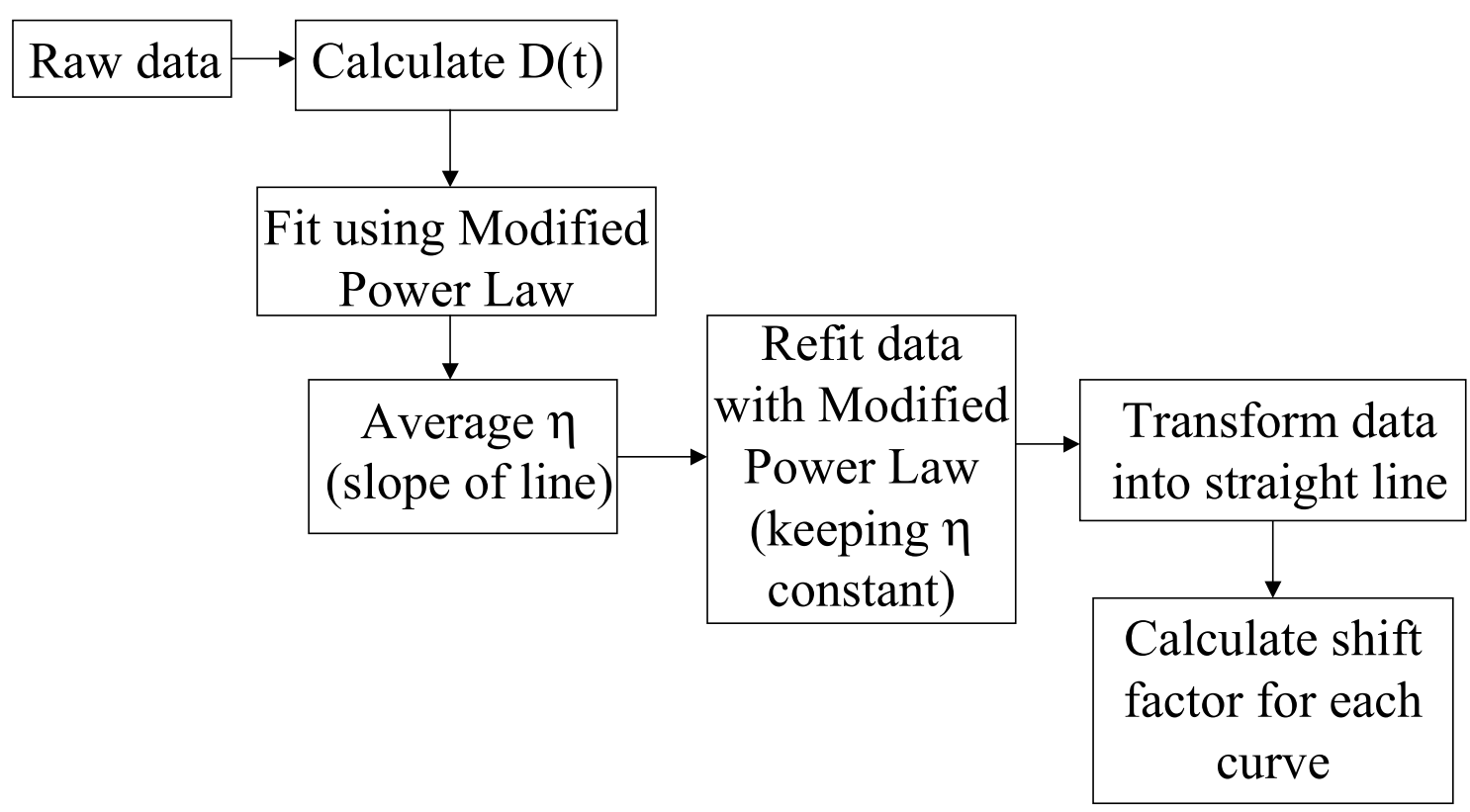


An aging study was done on three specimens separately. For each specimen, all of the snapshot curves were shifted to the longest age(hr). The three specimens had a slightly different longest age due to differences in the testing schedule. Therefore, all age factors for all snapshot tests in a given specimen were shifted so that $\mathrm{a}_{\mathrm{e}}=1$ corresponds to $t_{e}=15$ min. This was done for each specimen independently using its own $a_{e}\left(t_{e}\right)$ curve. Then the three specimens have the same reference age, $t_{e}=15$ min with $a_{e}=1$. Next, $a_{e}$ values of every snapshot for all specimens can be plotted together, Figs. 3.14 through 3.18. Linear regression of $\log \left(a_{\mathrm{e}}\right)$ vs. $\log \left(\mathrm{t}_{\mathrm{e}}\right)$ as in Figs. 3.14 through 3.18 yields a linear equation that allows us to predict the shift factor $\mathrm{a}_{\mathrm{e}}\left(\mathrm{t}_{\mathrm{e}}\right)$ for any age $\mathrm{t}_{\mathrm{e}}$, including the age of the full-size samples tested by Barbero and Rangaragjan [20]. The slope of the line is the aging parameter $\mu$. Tables 3.2 through 3.6 compare the values of $\mu$ for each aging study preformed for each material and give the standard deviation and coefficient of variation of each study with the master $\mu$. An aging study was preformed for materials A, B, and C at $21.1{ }^{\circ} \mathrm{C}$ to observe if temperature had any effect on the aging parameter, $\mu$. As can be seen in Tables 3.7 through 3.9 temperature has not effect on the aging parameter. 
Table 3.1. Typical testing schedule for an aging study.

\begin{tabular}{|c|c|c|c|}
\cline { 2 - 5 } \multicolumn{1}{c|}{} & day and time test began & $\mathbf{t}_{\mathbf{e}}$ ( $\left.\mathbf{m i n}\right)$ & test duration $(\mathbf{m i n})$ \\
\hline test 1 & $01 / 23 / 0210: 11: 25 \mathrm{AM}$ & 11.42 & 1.14 \\
\hline test 2 & $01 / 23 / 0210: 16: 39 \mathrm{AM}$ & 16.65 & 1.67 \\
\hline test 3 & $01 / 23 / 0210: 30: 13 \mathrm{AM}$ & 30.22 & 3.02 \\
\hline test 4 & $01 / 23 / 0211: 33: 23 \mathrm{AM}$ & 93.38 & 9.34 \\
\hline test 5 & $01 / 23 / 0202: 57: 41 \mathrm{PM}$ & 297.68 & 29.77 \\
\hline test 6 & $01 / 24 / 0209: 54: 18 \mathrm{AM}$ & 1434.30 & 143.43 \\
\hline test 7 & $01 / 25 / 0209: 53: 56 \mathrm{AM}$ & 2873.93 & 287.39 \\
\hline test 8 & $01 / 26 / 0201: 12: 58 \mathrm{PM}$ & 4512.97 & 451.30 \\
\hline
\end{tabular}

Table 3.2. Comparison of aging studies of material A.

\begin{tabular}{|ccc|}
\hline & $\boldsymbol{\mu}=$ & $\mathbf{0 . 4 8 1}$ \\
\hline specimen & $\boldsymbol{\mu}$ & \% diff \\
\hline $\boldsymbol{A 1}$ & 0.510 & 6.09 \\
\hline $\boldsymbol{A 2}$ & 0.481 & 0.02 \\
\hline $\boldsymbol{A 3}$ & 0.465 & 3.31 \\
\hline $\boldsymbol{A 4 1}$ & 0.490 & 1.96 \\
\hline $\boldsymbol{A 4 2}$ & 0.492 & 2.38 \\
\hline average & 0.488 \\
\hline standard deviation & 0.0166 \\
\hline coefficient of variation & $3.40 \%$ \\
\hline
\end{tabular}

Table 3.3. Comparison of aging studies of material $B$.

\begin{tabular}{|c|c|c|}
\hline \multicolumn{3}{|c|}{$\mu=0.720$} \\
\hline specimen & $\mu$ & $\%$ diff \\
\hline B1 & 0.717 & 0.47 \\
\hline B2 & 0.722 & 0.20 \\
\hline B3 & 0.728 & 1.05 \\
\hline \multicolumn{3}{|c|}{ average 0.722} \\
\hline \multicolumn{3}{|c|}{ standard deviation 0.0055} \\
\hline coefficient of va & $0.76 \%$ & \\
\hline
\end{tabular}


Table 3.4. Comparison of aging studies of material C.

\begin{tabular}{|ccc|}
\hline \multicolumn{3}{|c|}{$\boldsymbol{\mu}=\mathbf{0 . 7 1 8}$} \\
\hline specimen & $\boldsymbol{\mu}$ & \% diff \\
\hline $\mathbf{C 2}$ & 0.709 & 1.15 \\
\hline C3 & 0.712 & 0.76 \\
\hline C4 & 0.678 & 5.59 \\
\hline C5 & 0.793 & 10.57 \\
\hline C6 & 0.636 & 11.40 \\
\hline average & 0.706 \\
\hline standard deviation & 0.0580 \\
\hline coefficient of variation & $8.21 \%$ \\
\hline
\end{tabular}

Table 3.5. Comparison of aging studies of material D.

\begin{tabular}{|c|c|c|}
\hline$\mu=$ & 0.177 & \\
\hline specimen & $\mu$ & $\%$ diff \\
\hline D1 & 0.196 & 10.78 \\
\hline$D 2$ & 0.169 & 4.27 \\
\hline D3 & 0.169 & 4.46 \\
\hline average & 0.178 & \\
\hline standard deviation & 0.0154 & \\
\hline coefficient of variation & $8.69 \%$ & \\
\hline
\end{tabular}

Table 3.6. Comparison of aging studies of material $\mathbf{E}$.

\begin{tabular}{|ccc|}
\hline & $\mu=$ & $\mathbf{0 . 6 0 2}$ \\
\hline specimen & $\boldsymbol{\mu}$ & \% diff \\
\hline E1 & 0.592 & 1.69 \\
\hline E2 & 0.496 & 17.63 \\
\hline E3 & 0.698 & 15.92 \\
\hline E5 & 0.551 & 8.41 \\
\hline E6 & 0.630 & 4.65 \\
\hline E7 & 0.605 & 0.47 \\
\hline average & 0.577 & 4.19 \\
\hline standard deviation & 0.1010 & \\
\hline coefficient of variation & $16.97 \%$ \\
\hline
\end{tabular}


Table 3.7. Comparison of master $\mu$ at $21.1^{\circ} \mathrm{C}$ and $40{ }^{\circ} \mathrm{C}$ for material A.

\begin{tabular}{|c|c|}
\hline $\mathrm{T}^{\circ} \mathrm{C}$ & $\mu$ \\
\hline 40 & 0.481 \\
\hline 21.1 & 0.519 \\
\hline average & 0.500 \\
\hline standard deviation & 0.027 \\
\hline coefficient of variation & $5.42 \%$ \\
\hline
\end{tabular}

Table 3.8. Comparison of master $\mu$ at $21.1^{\circ} \mathrm{C}$ and $40{ }^{\circ} \mathrm{C}$ for material $\mathrm{B}$.

\begin{tabular}{|c|c|}
\hline $\mathrm{T}^{\circ} \mathrm{C}$ & $\mu$ \\
\hline 40 & 0.720 \\
\hline 21.1 & 0.717 \\
\hline average & 0.719 \\
\hline standard deviation & 0.003 \\
\hline coefficient of variation & $0.35 \%$ \\
\hline
\end{tabular}

Table 3.9. Comparison of master $\mu$ at $21.1^{\circ} \mathrm{C}$ and $40{ }^{\circ} \mathrm{C}$ for material $\mathrm{C}$.

\begin{tabular}{|c|c|}
\hline $\mathrm{T}^{\circ} \mathrm{C}$ & $\mu$ \\
\hline 40 & 0.718 \\
\hline 21.1 & 0.634 \\
\hline average & 0.676 \\
\hline standard deviation & 0.059 \\
\hline coefficient of variation & $8.80 \%$ \\
\hline
\end{tabular}




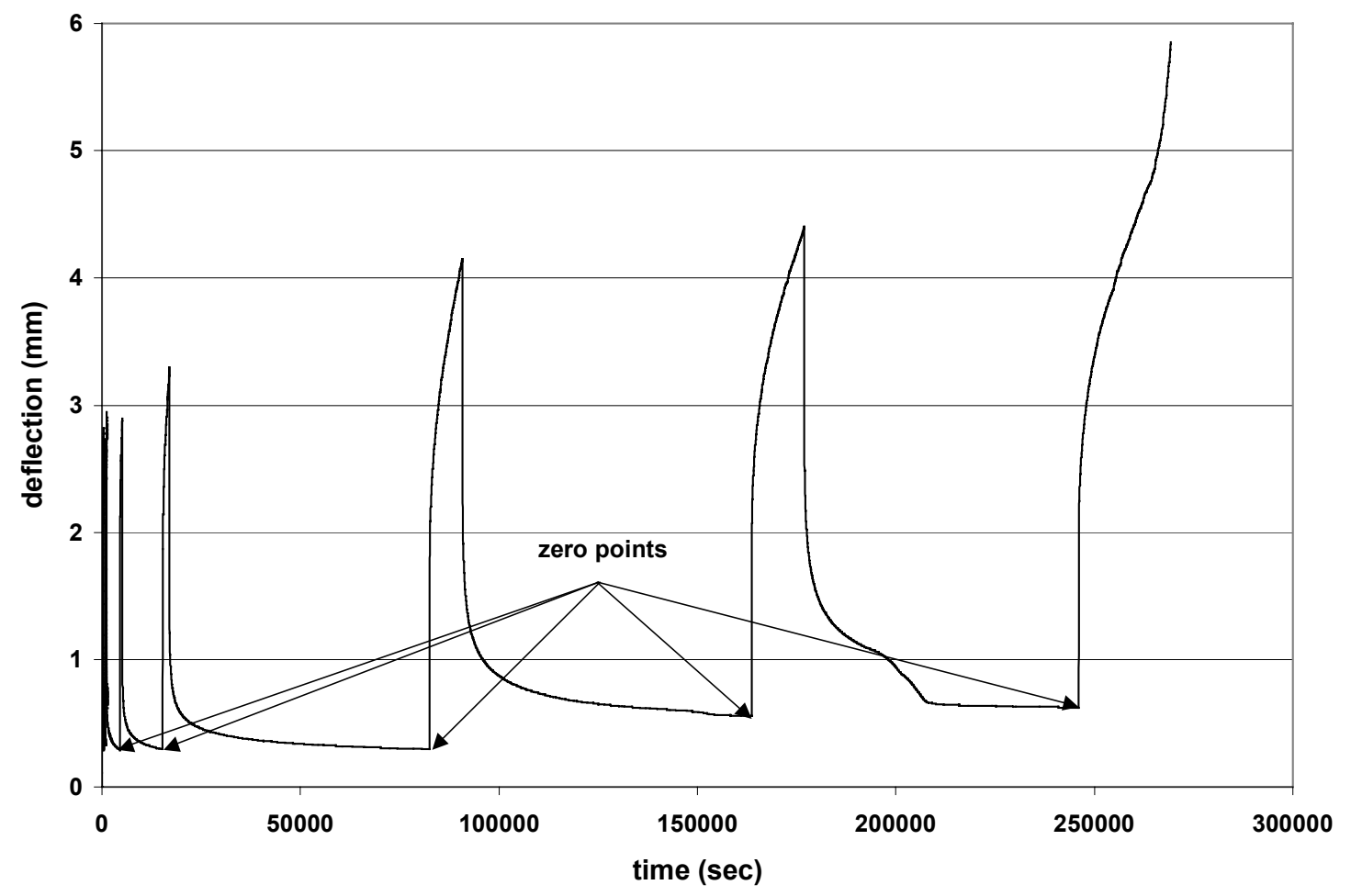

Figure 3.1. Deflection versus time for aging study test.

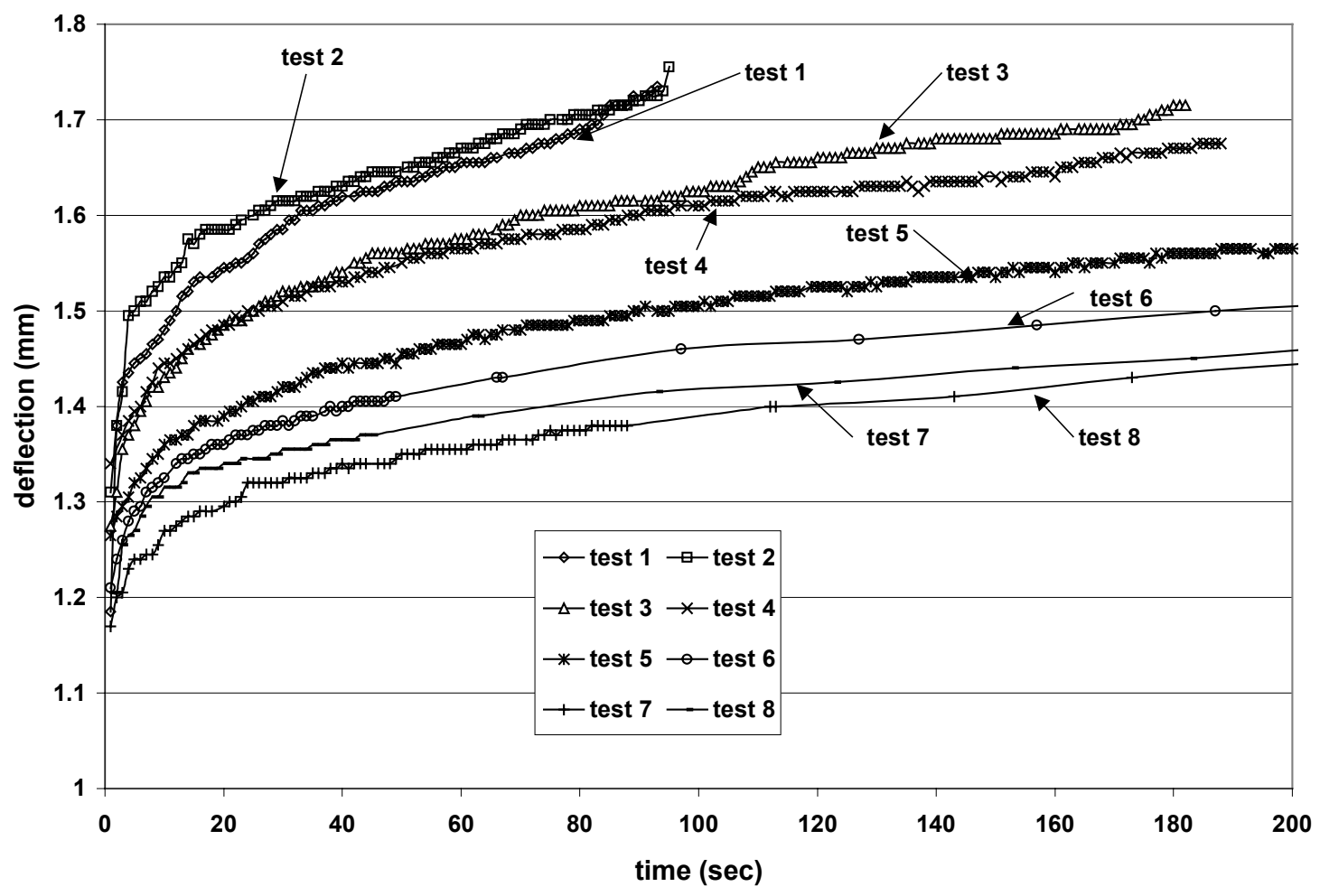

Figure 3.2. Illustration of the reasoning for mechanical conditioning. 


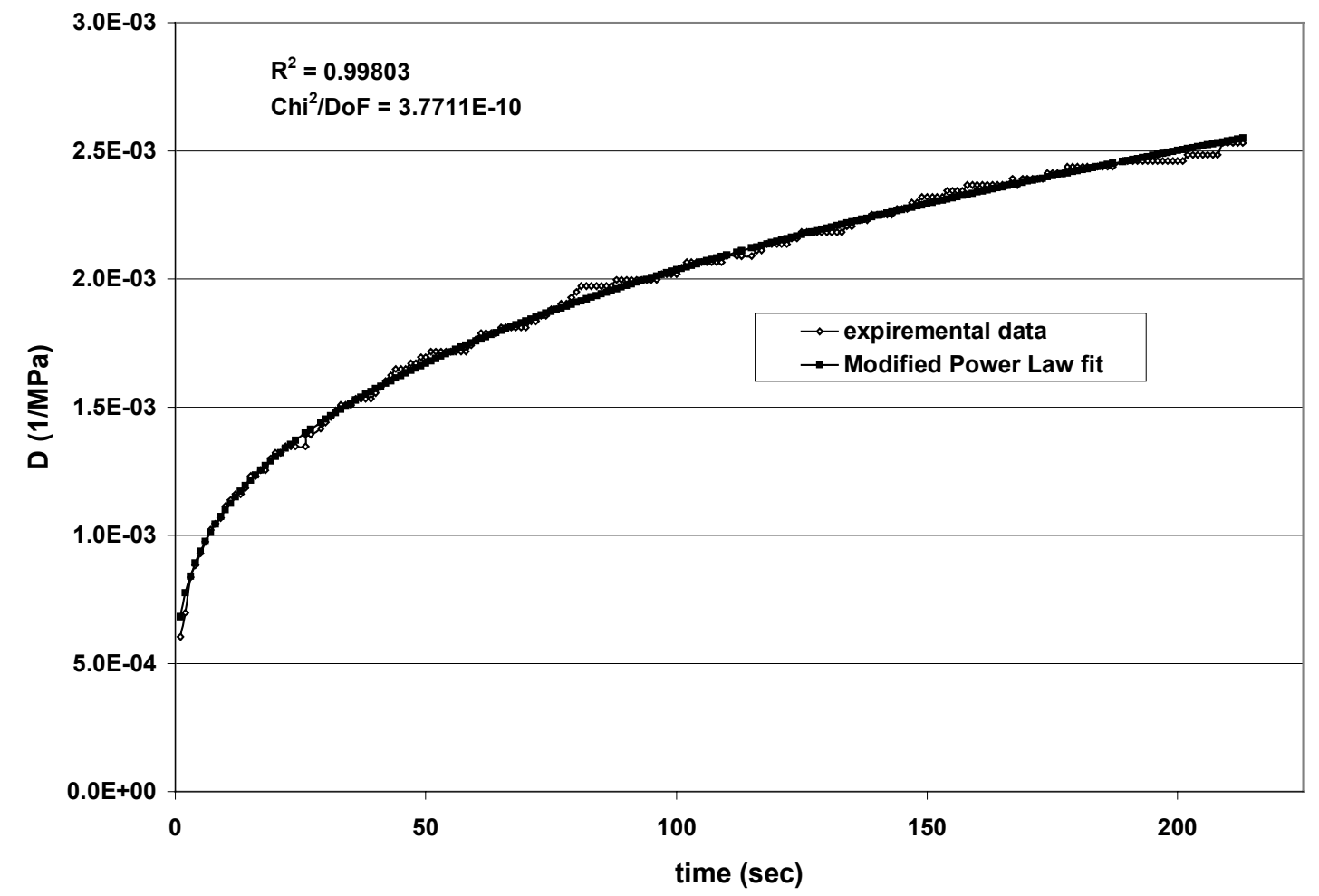

Figure 3.3. Fit of the data using the Modified Power Law.

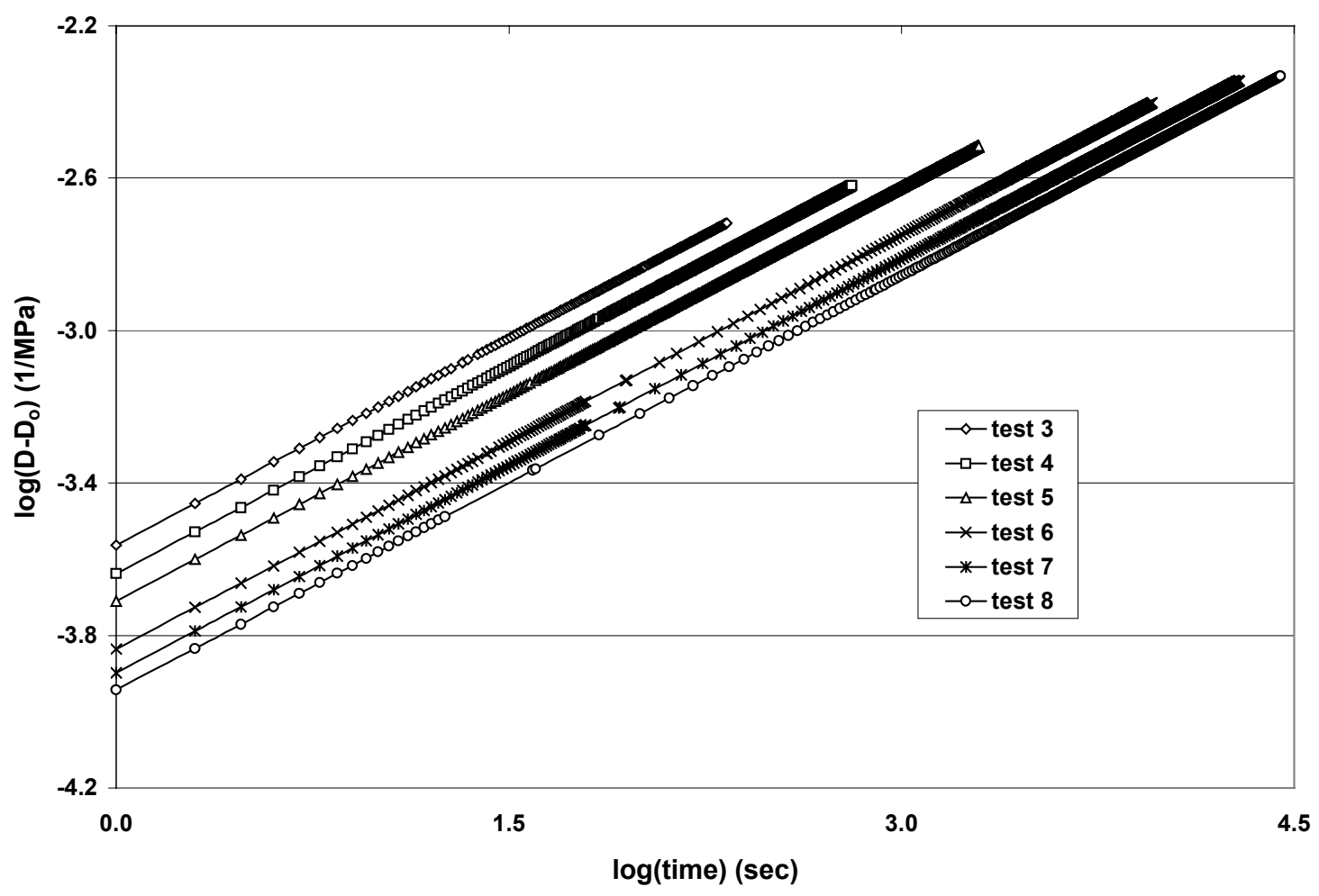

Figure 3.4. Aging study data transformed into the Power Law for material A. 


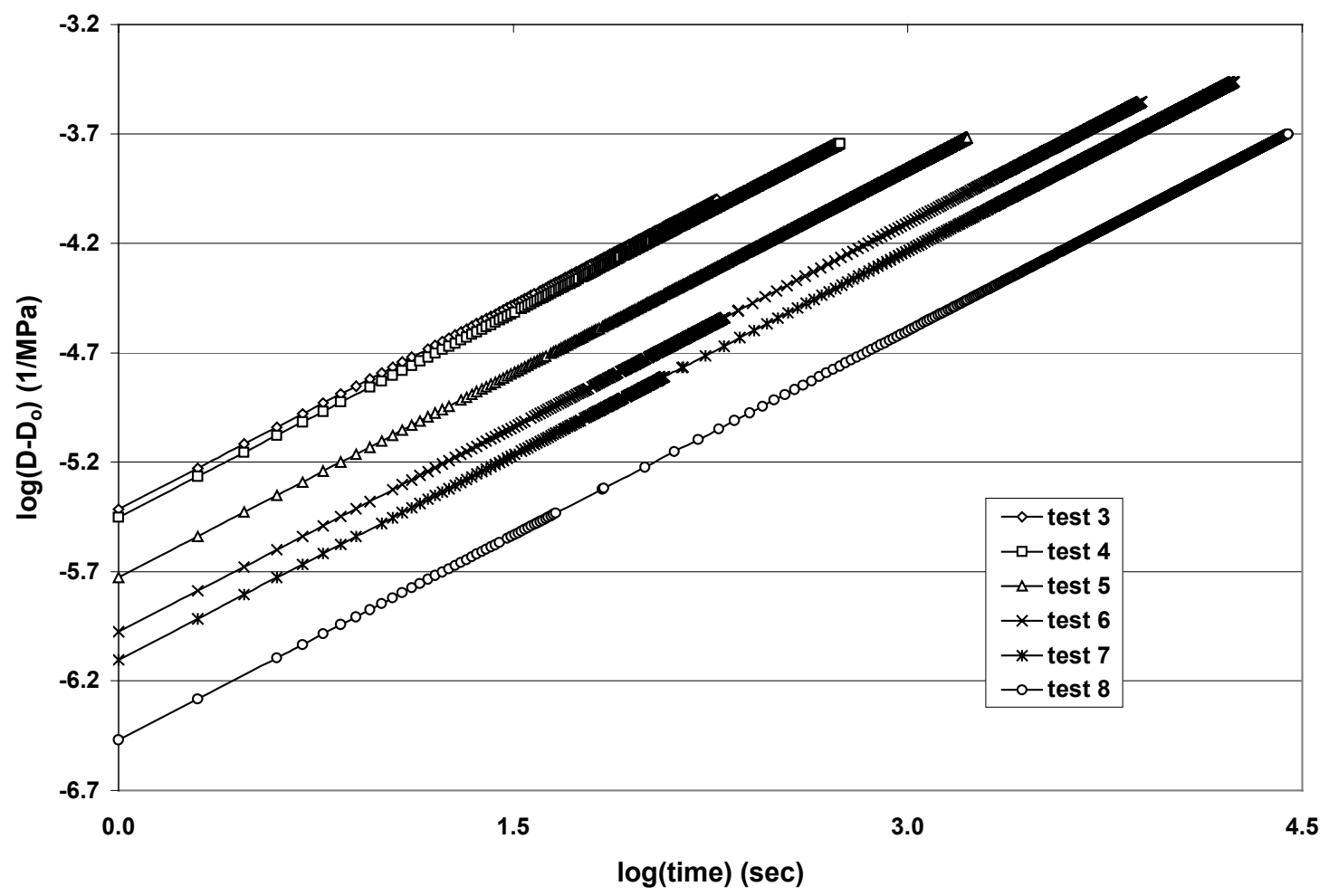

Figure 3.5. Aging study data transformed into the Power Law for material B.

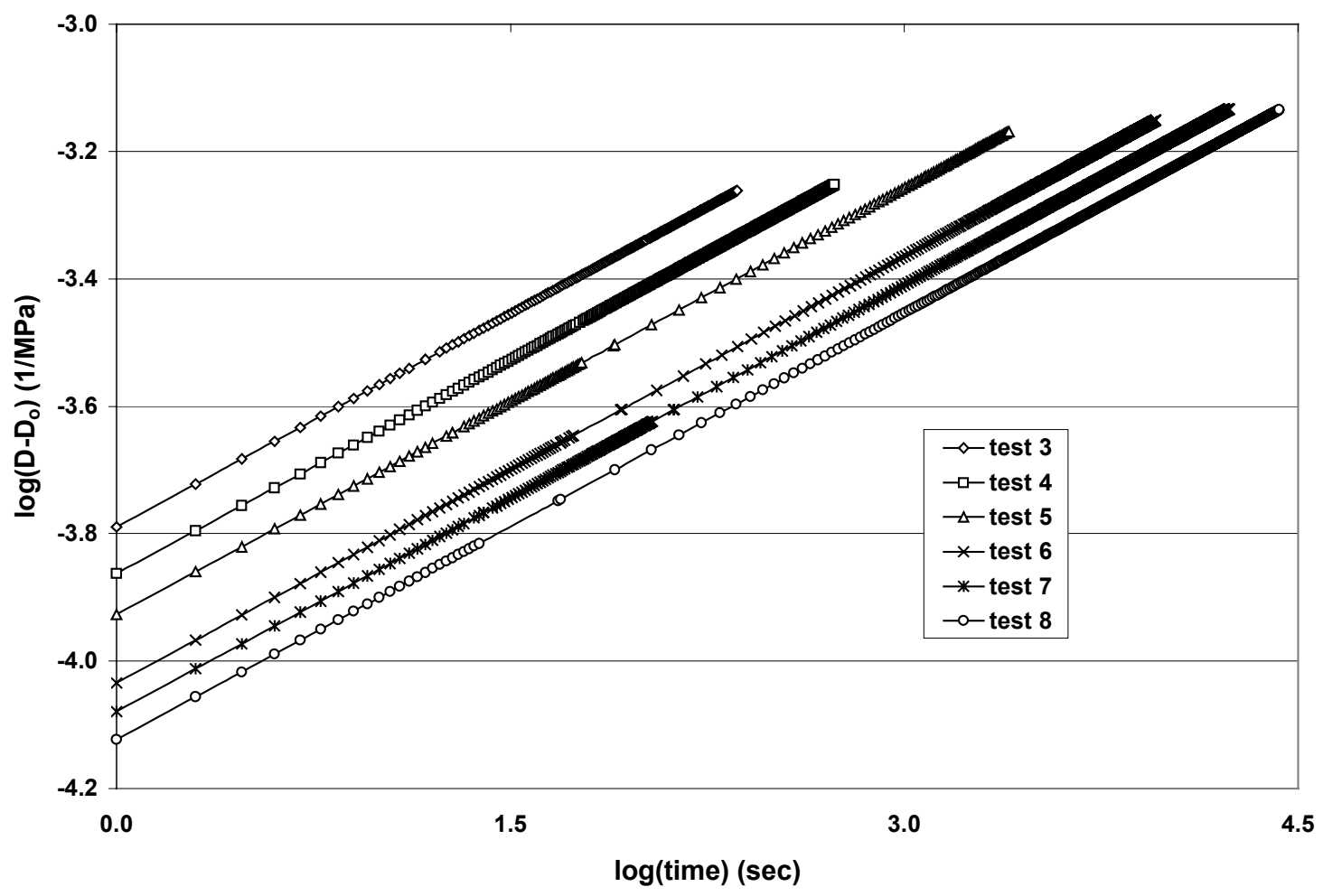

Figure 3.6. Aging study data transformed into the Power Law for material C. 


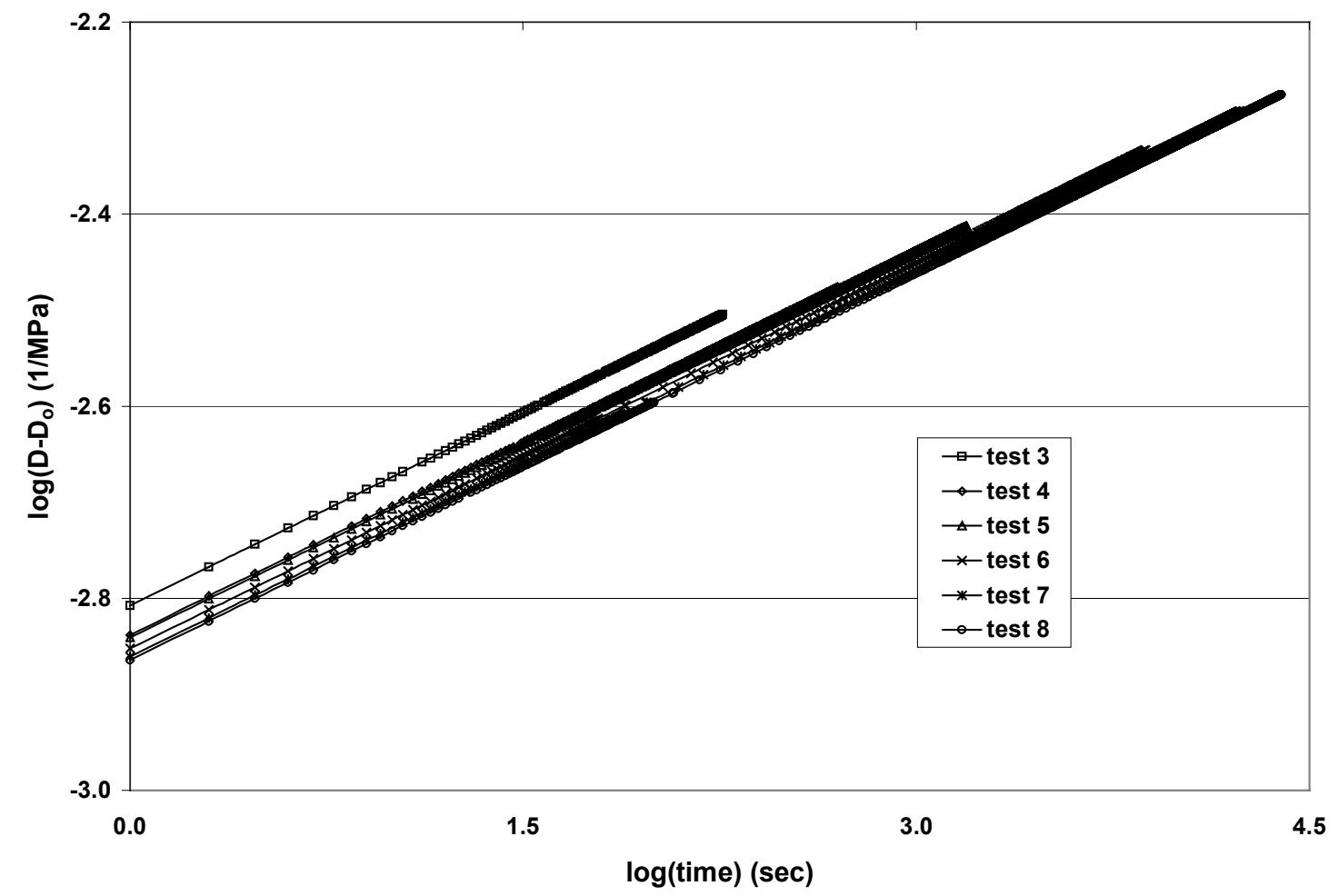

Figure 3.7. Aging study data transformed into the Power Law for material D.

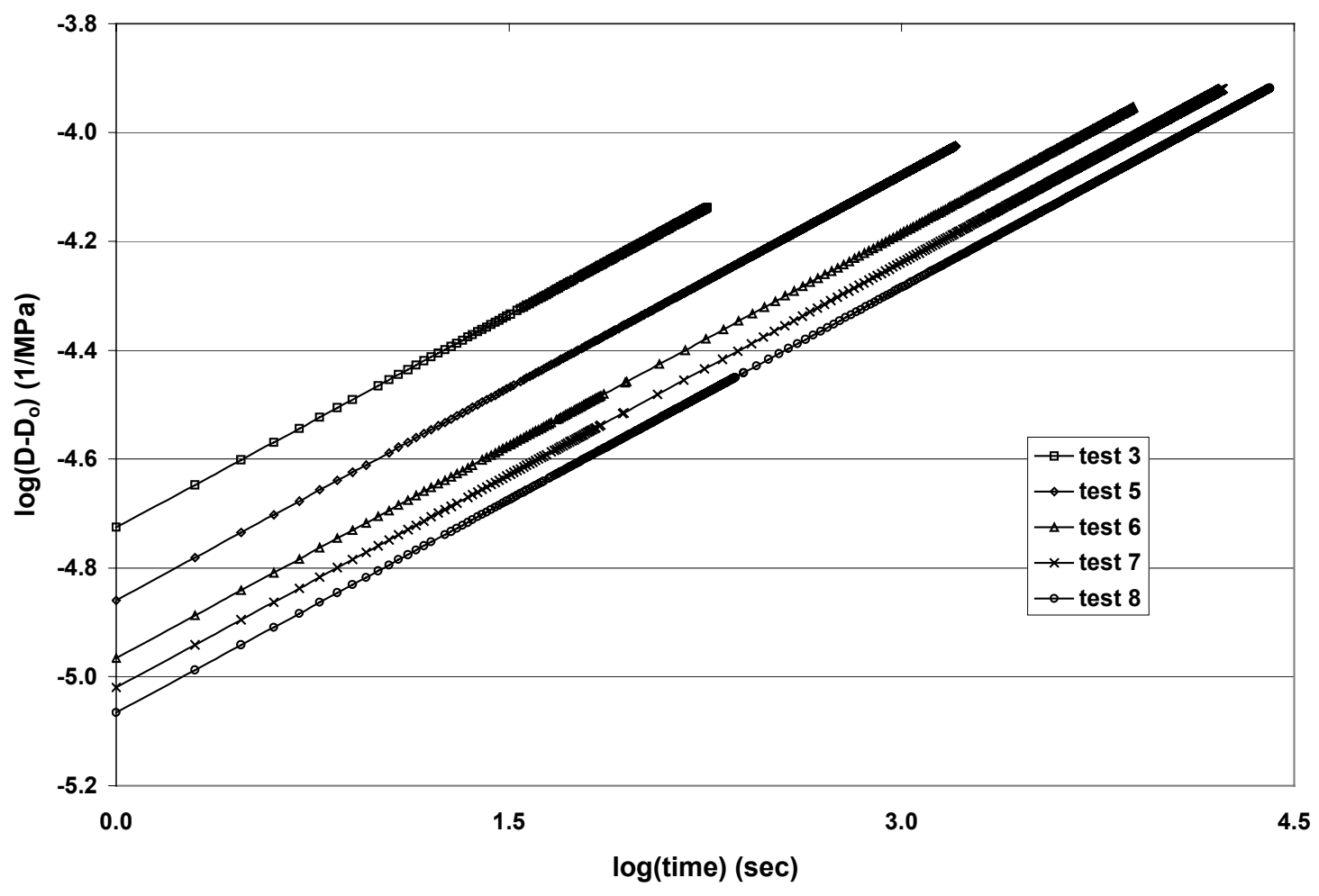

Figure 3.8. Aging study data transformed into the Power Law for material $E$. 


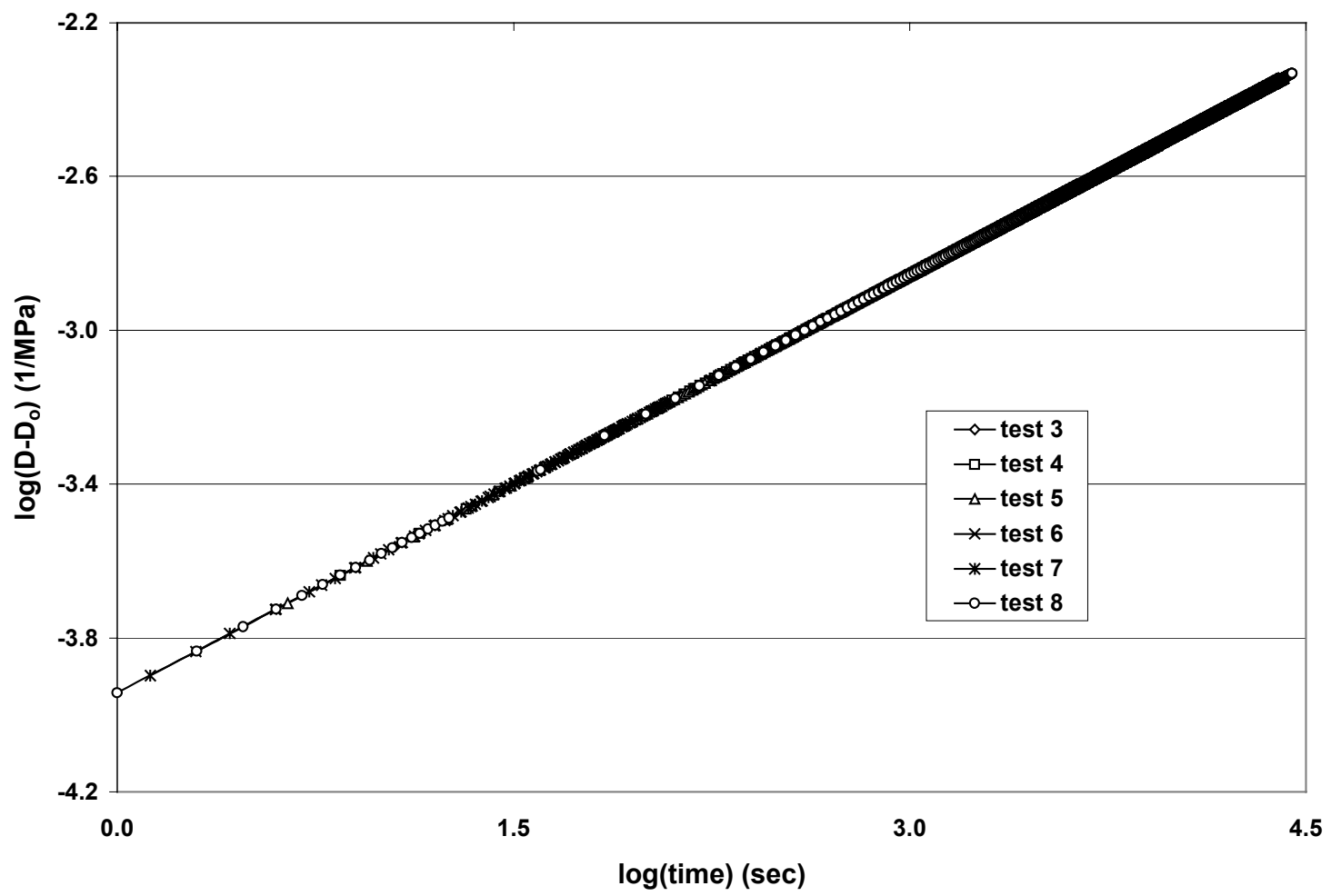

Figure 3.9. Aging study data shifted to obtain $\mu$ for material A.

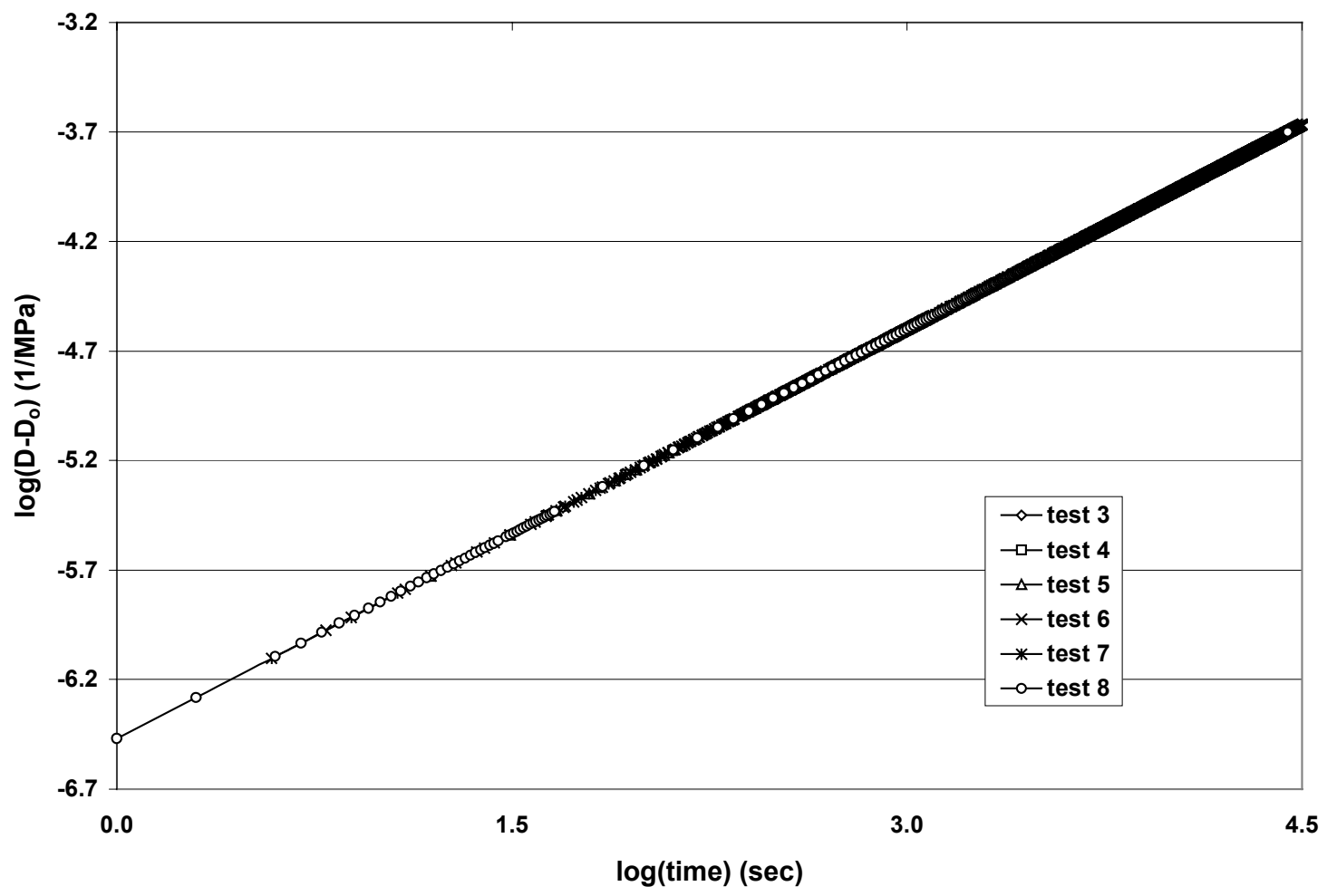

Figure 3.10. Aging study data shifted to obtain $\mu$ for material $B$. 


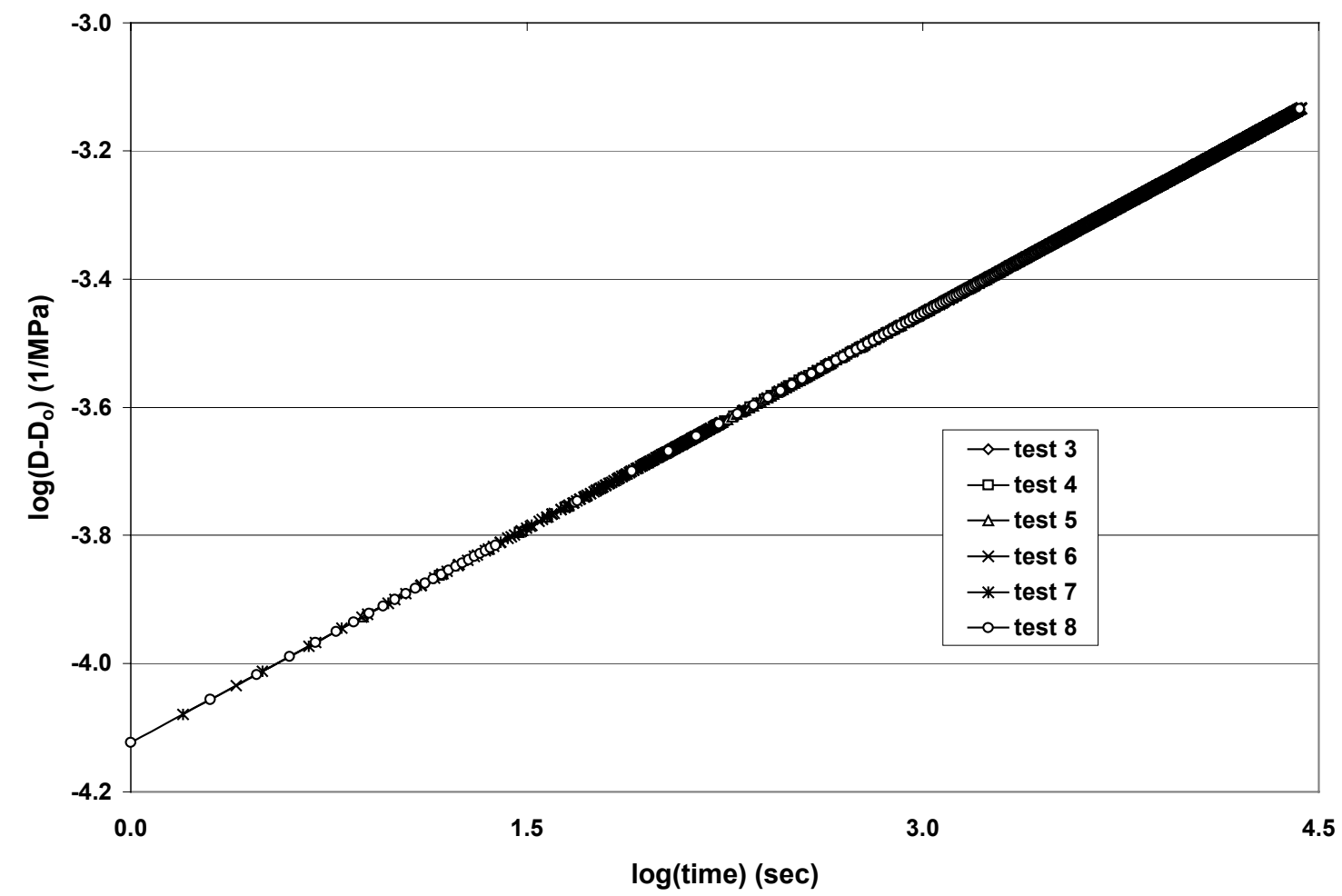

Figure 3.11. Aging study data shifted to obtain $\mu$ for material C.

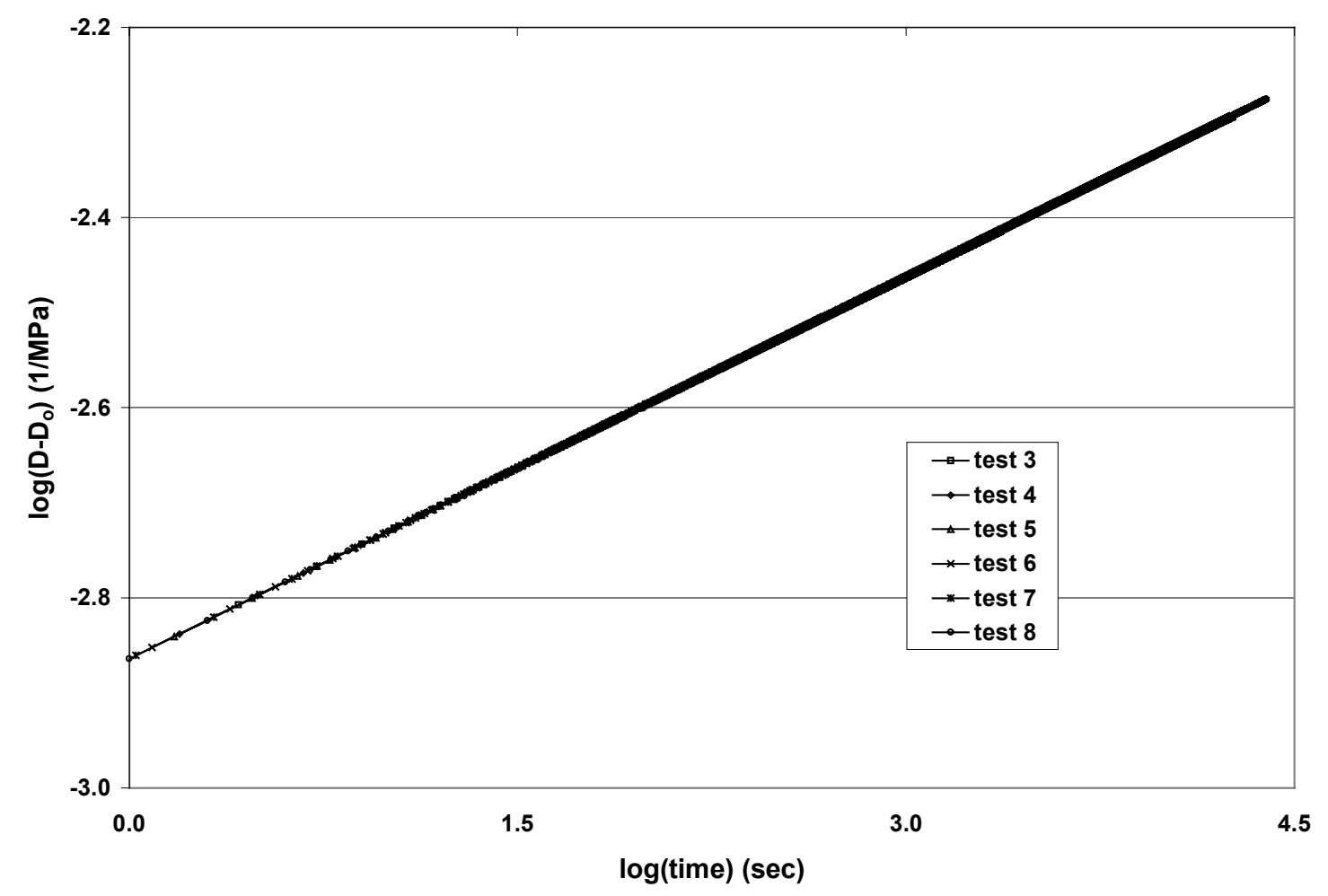

Figure 3.12. Aging study data shifted to obtain $\mu$ for material $D$. 


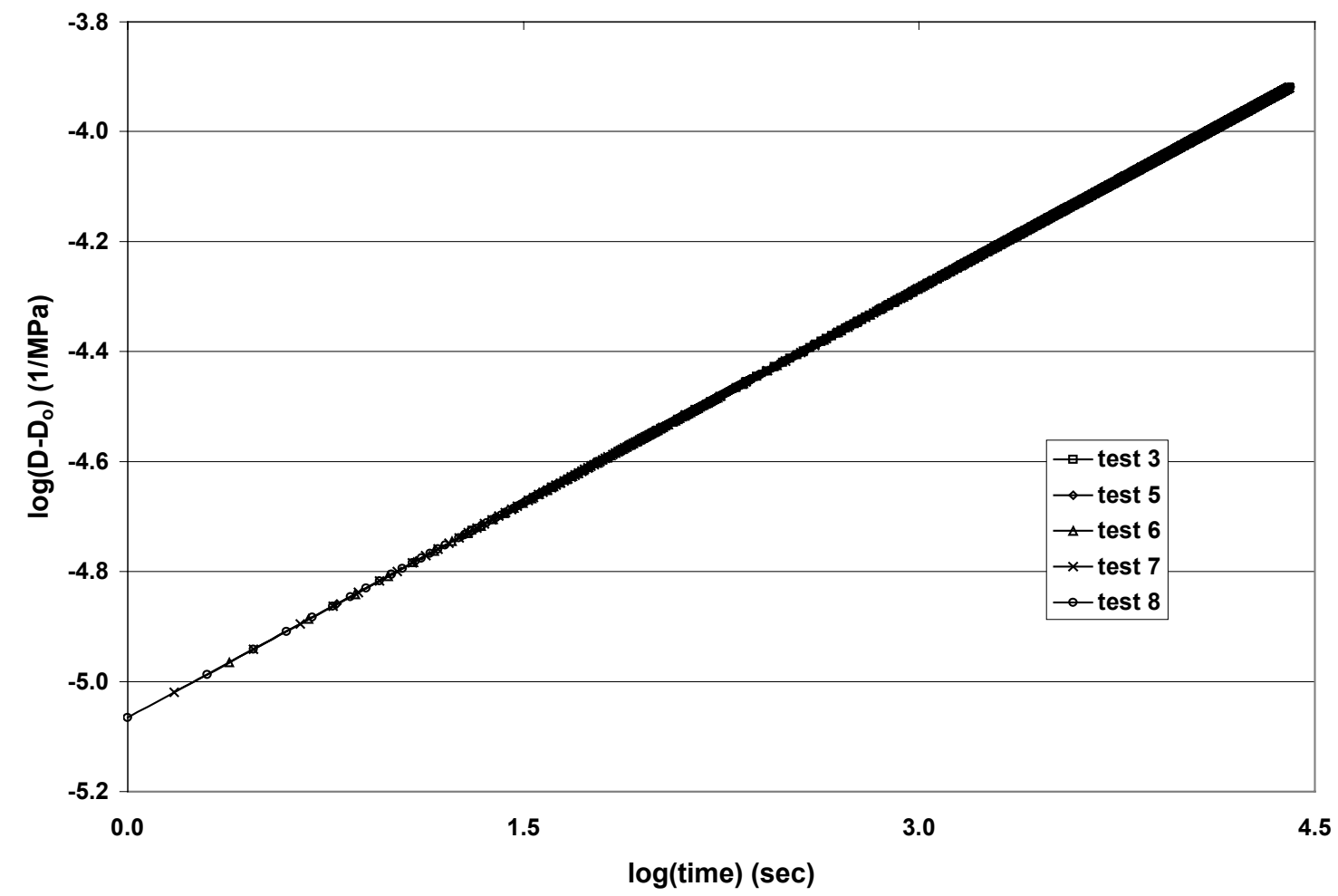

Figure 3.13. Aging study data shifted to obtain $\mu$ for material E.

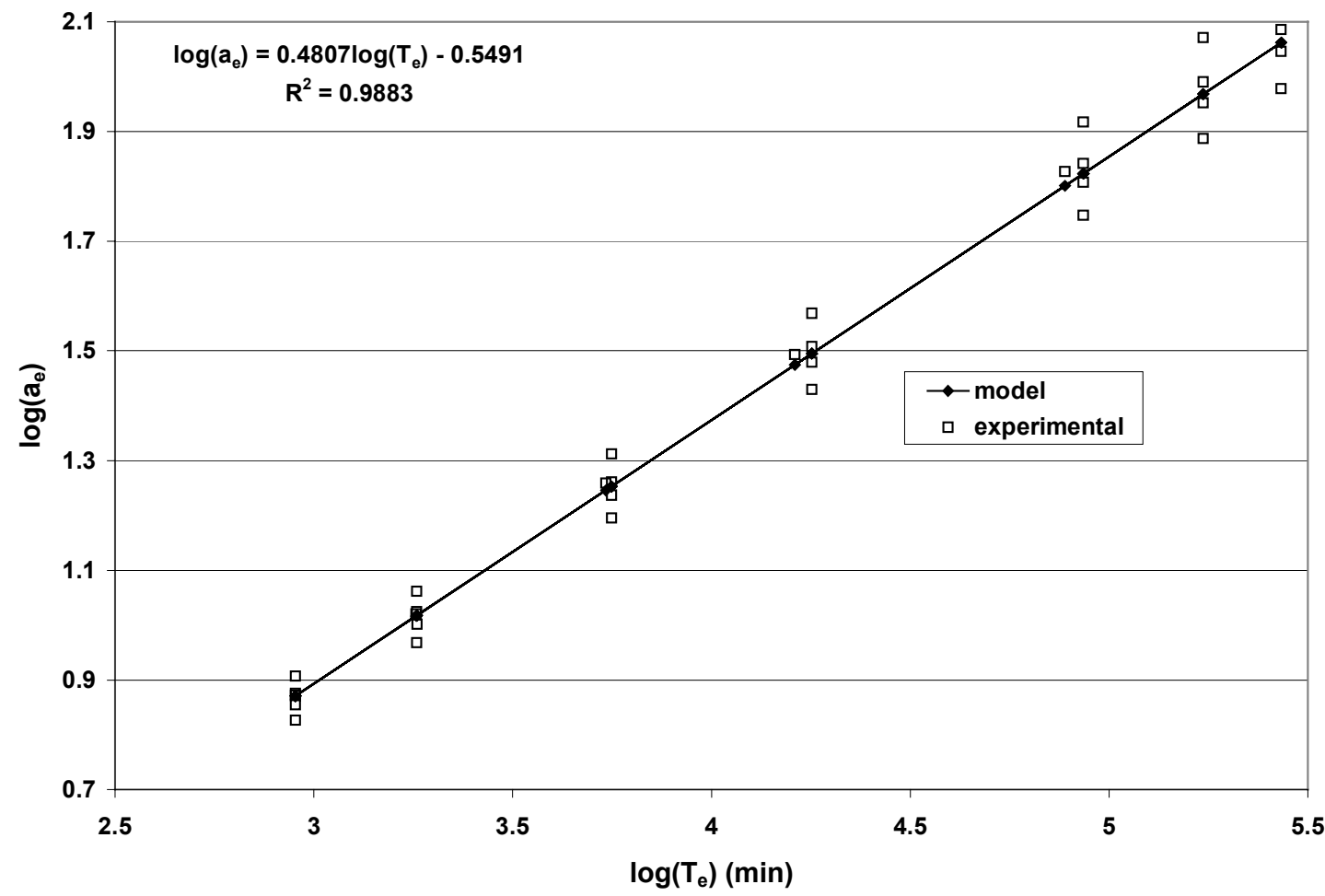

Figure 3.14. Theoretical and experimental values of the aging shift factors for material A 


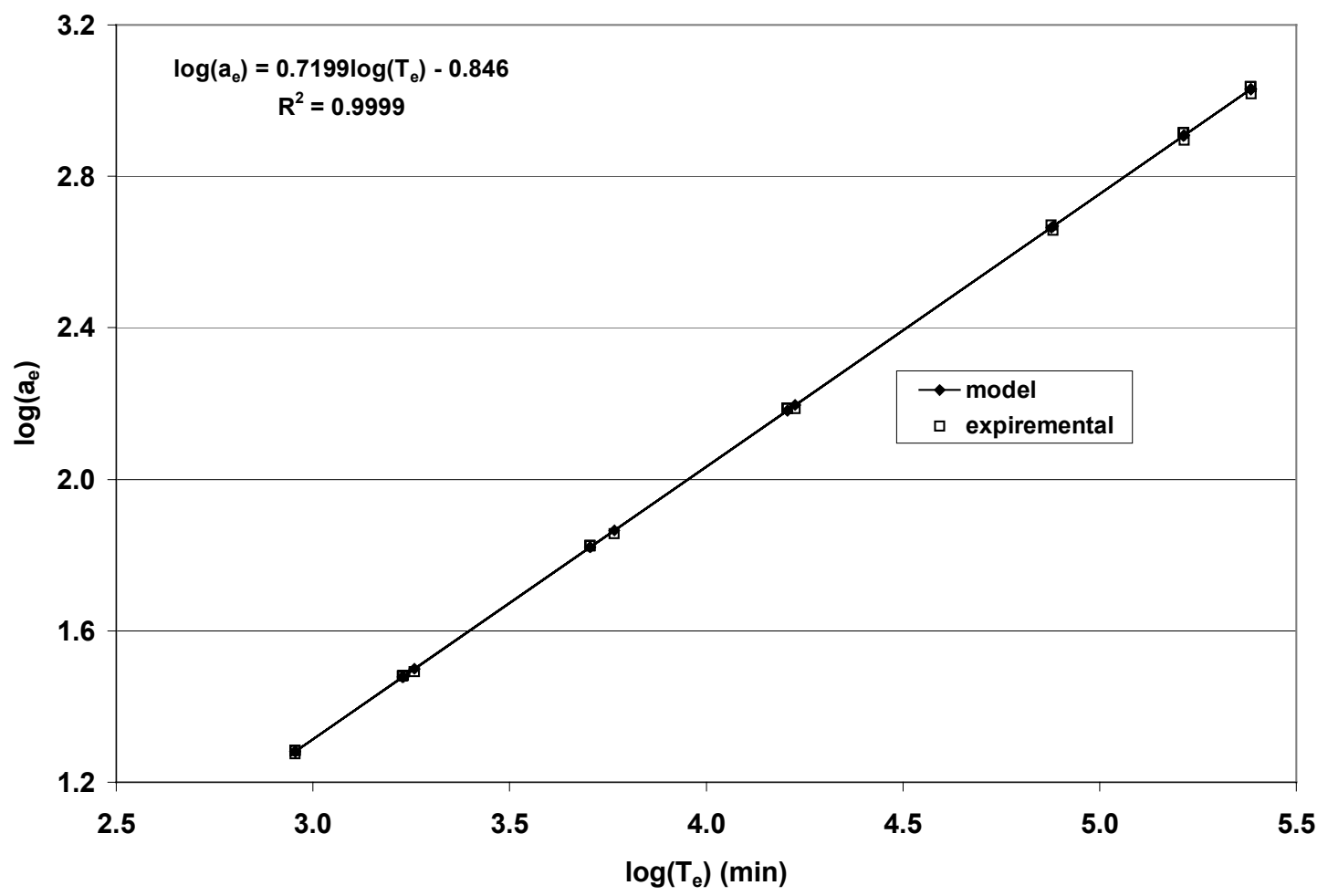

Figure 3.15. Theoretical and experimental values of the aging shift factors for material B.

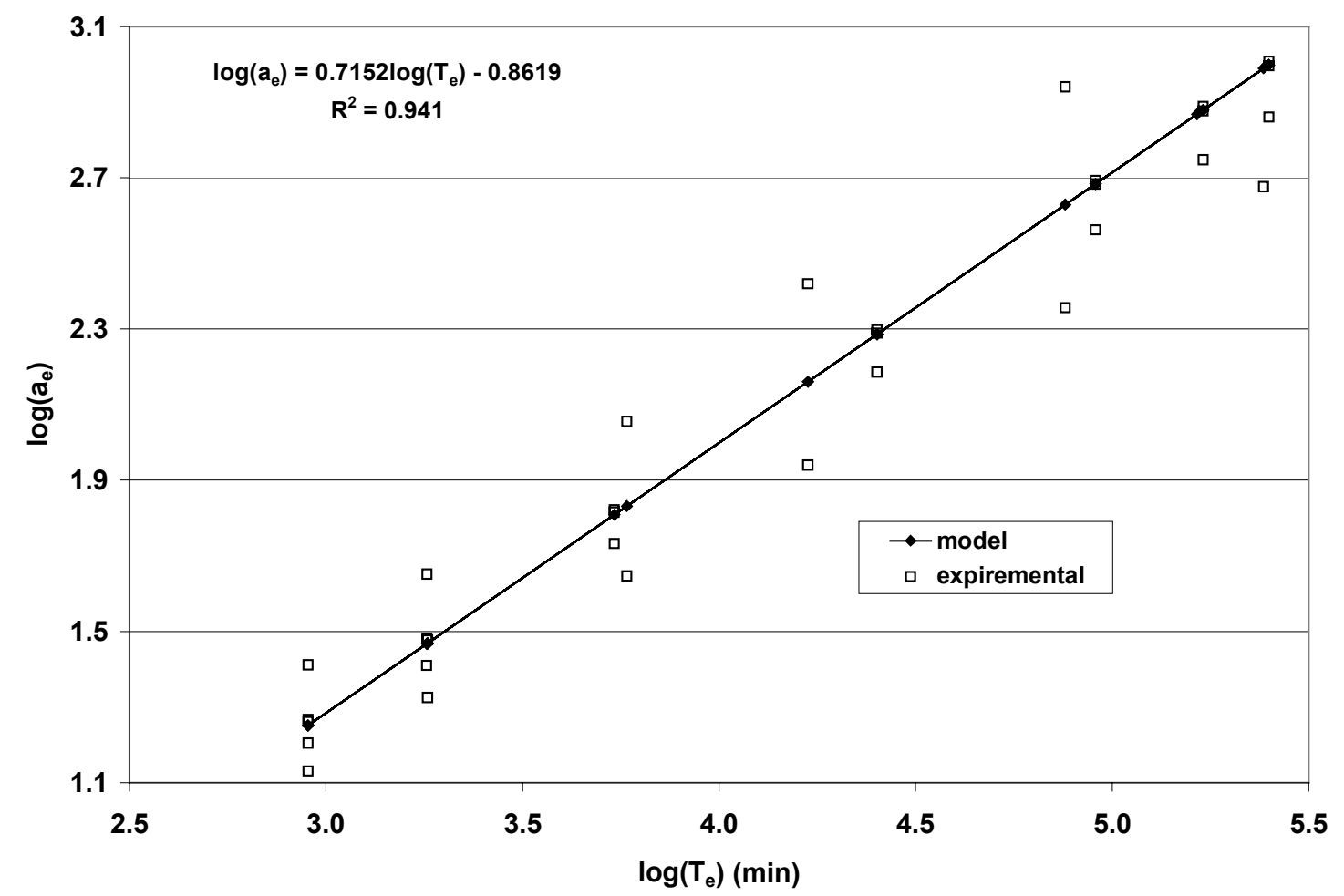

Figure 3.16. Theoretical and experimental values of the aging study shift factors for material C. 


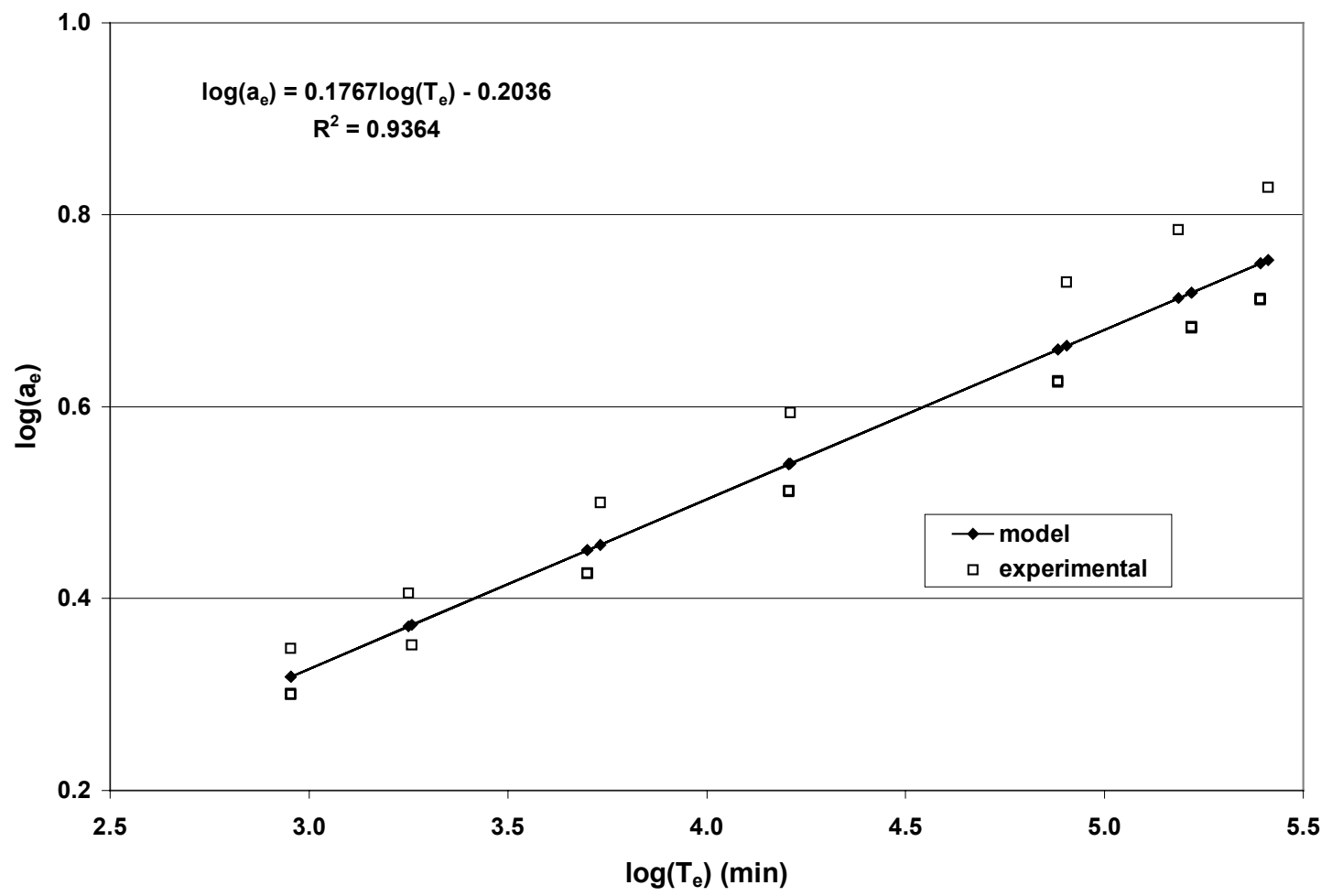

Figure 3.17. Theoretical and experimental values of the aging shift factors for material D.

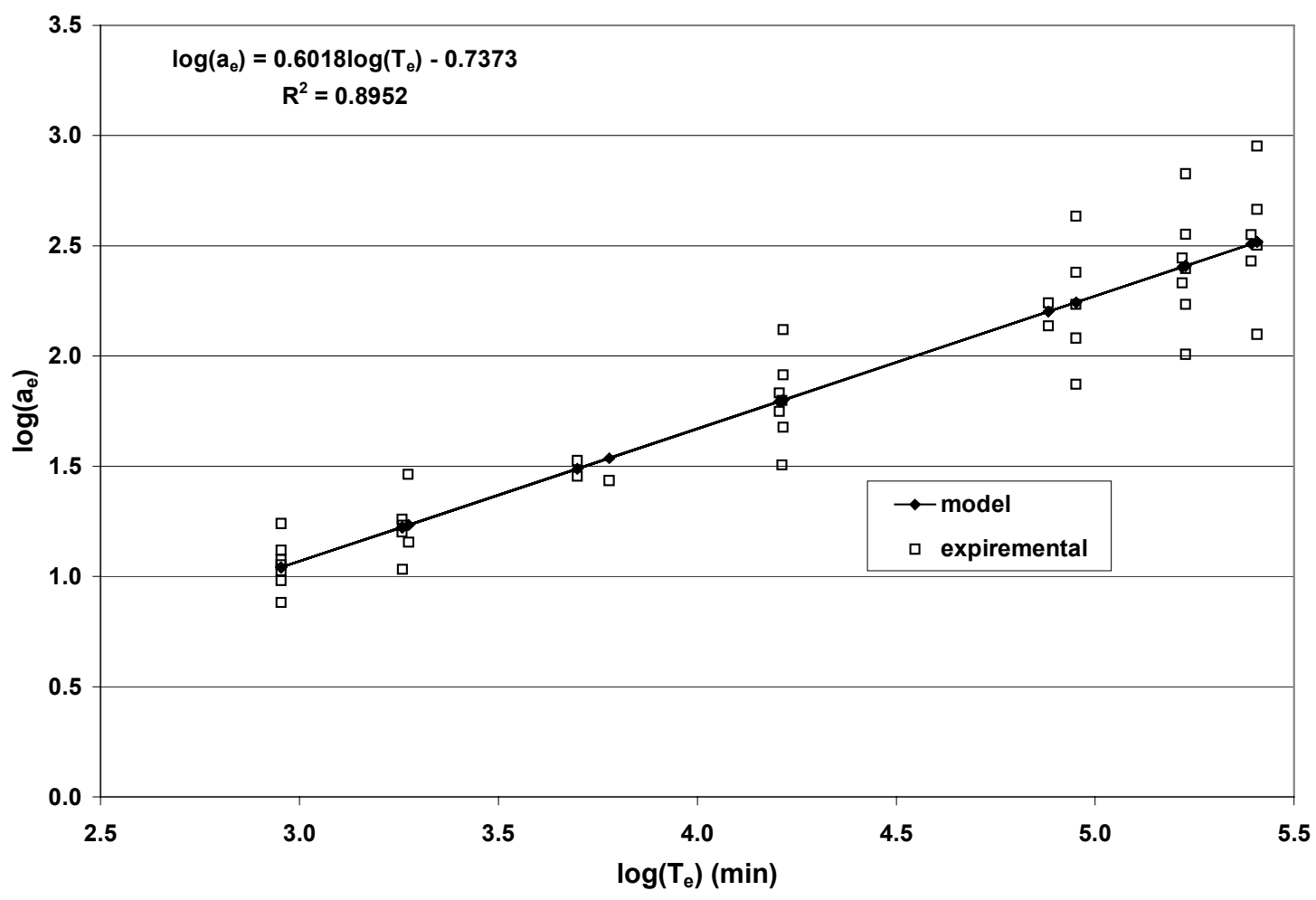

Figure 3.18. Theoretical and experimental values of the aging shift factors for material E. 


\section{CHAPTER 4 TIME-TEMPERATURE SUPERPOSITION PRINCIPLE}

\subsection{Introduction}

The time temperature superposition principle (TTSP) is a method used to predict the behavior of a material for times longer than the test time. This method accelerates the creep of the material by an increase in the temperature. It assumes that this increase in temperate can accelerate but not change the material property being investigated, in our case compliance.

Creep compliance is measured at each temperature over a span of time shorter than $t_{\mathrm{e}} / 10$ so that the curve is free of physical aging effects. Test data at each temperature plotted on a base-10 log-log scale versus time. One temperature is chosen as a reference temperature. The remaining curves are superimposed onto the reference curve by a shifting with a horizontal shift factor $\mathrm{a}_{\mathrm{T}}$. Once all of the curves are shifted, a master curve that spans longer time than the actual testing time is created. This master curve can then be shifted to any temperature, and used to predict the value of compliance over time. Since every individual test is free of physical aging, the master curve is free of physical aging even if it spans a time range much larger than $\mathrm{t}_{\mathrm{e}} / 10$.

\subsection{Testing Procedure}

For TTSP testing the materials were prepared as described in Section 2.2.3. They were then placed in the testing fixture at the desired testing temperature to let them age. Each specimen was aged $60 \mathrm{~min}$ and then tested for $6 \mathrm{~min}$, following the snapshot assumption to avoid the effects of physical aging. During the aging process, two oneminute tests were preformed at $t_{e}=20 \mathrm{~min}$ and $t_{e}=25 \mathrm{~min}$. This mechanically conditioned the specimen as described in Section 3.3 before the actual test at $\mathrm{t}_{\mathrm{e}}=60 \mathrm{~min}$. 


\subsection{Data Analysis}

Three specimens of each material were tested across the temperature range, approximately $21.1{ }^{\circ} \mathrm{C}$ to $57{ }^{\circ} \mathrm{C}$ for each material. Next, the log base- 10 of the time and compliance was calculated and plotted for each material. The lowest temperature was used as a reference temperature. Data was then superimposed on to this curve using a horizontal shift factor, $\mathrm{a}_{\mathrm{T}}$.

The curves were shifted by minimizing the difference in shifted time $\left(t_{r e f}-t_{i} / a_{T i}\right)$, where $\mathrm{t}_{\mathrm{ref}}$ is the reference time, $\mathrm{t}_{\mathrm{i}}$ is the time, and $\mathrm{a}_{T \mathrm{i}}$ is the shift factor, between the two curves on a base-10 log-log scale. Testing temperatures were chosen so that the compliances would overlap. For the materials in this study, it was found that a change every 5 to $7{ }^{\circ} \mathrm{C}$ was sufficient. A temperature schedule was formulated for testing at intervals of about 5 to $7{ }^{\circ} \mathrm{C}$ apart. The testing temperature did not exceed the $\mathrm{T}_{\mathrm{g}}$ of the material. Three specimens were tested at or near the nominal temperature in the testing schedule. The data sets were designated $\mathrm{T}_{1 \mathrm{a}}, \mathrm{T}_{1 \mathrm{~b}}, \mathrm{~T}_{1 \mathrm{c}}$ through $\mathrm{T}_{\mathrm{nc}}$ where $\mathrm{n}$ is the number of different temperatures tested. Then all the data sets were shifted until they overlap with the data of the previous temperature, taking the lowest temperature as a reference temperature $\left(a_{\mathrm{T}}=1\right)$. Due to material variability and experimental error each set of data at a particular temperature did not lie on top of each other. In order to correct for this the following method was used to shift the data:

1. Data for each curve at the lowest tested temperature $T_{1 a}, T_{1 b}$, and $T_{1 c}$ were used as a reference temperature $\left(\mathrm{a}_{\mathrm{T}}=1\right)$. 
2. One curve at the next (higher) temperature $\mathrm{T}_{\mathrm{ia}}$, where $\mathrm{i}$ is the number of the data set being shifted, was then shifted to each previous curve $\mathrm{T}_{(\mathrm{i}-1) \mathrm{a}}$, $\mathrm{T}_{(\mathrm{i}-1) \mathrm{b}}, \mathrm{T}_{(\mathrm{i}-1) \mathrm{c}}$ producing three shift factors.

3. This is then done for the next two curves $T_{i b}$ and $T_{i c}$ producing six more shift factors.

4. The shift factors are then averaged giving one shift factor for the data at $\mathrm{T}_{\mathrm{i}}$.

5. Data for $\mathrm{T}_{\mathrm{ia}}, \mathrm{T}_{\mathrm{ib}}$, and $\mathrm{T}_{\mathrm{ic}}$ are then shifted using the averaged shift factor, $\mathrm{a}_{\mathrm{T}}$. In the case of material $\mathrm{D}$ it was noticed that each set of data was a straight line on $\log$ base-10 scale. This allowed the data to be fit with a Power Law equation to represent the master curve. To get the equation of the TTSP curve at a particular temperature the data for each specimen tested at that temperature was fit with a Power Law. The parameters of each of these curve fits were then averaged. Material E was tested using a Dynamic Mechanical Analyzer (DMA). This was done to avoid moving the material between tests. It was observed that the composition of the material was not uniform and a change in the material's location on the support and loading nodes produced varied results. Testing the material on the DMA allowed the material to be annealed and quenched without having to the move the specimen. Data for the TTSP of material E was obtained from Barbero and Julius [17]. The moving average of the master curve of each material was then taken to reduce the number of data points. The master curve of each material is shown in Figs. 4.1 through 4.5. Linear regression of $\log \left(\mathrm{a}_{\mathrm{T}}\right)$ vs. temperature as in Figs. 4.6 through 4.10 yields a linear equation that can be used to predict the shift factor $\mathrm{a}_{\mathrm{T}}(\mathrm{T})$ for any temperature. 


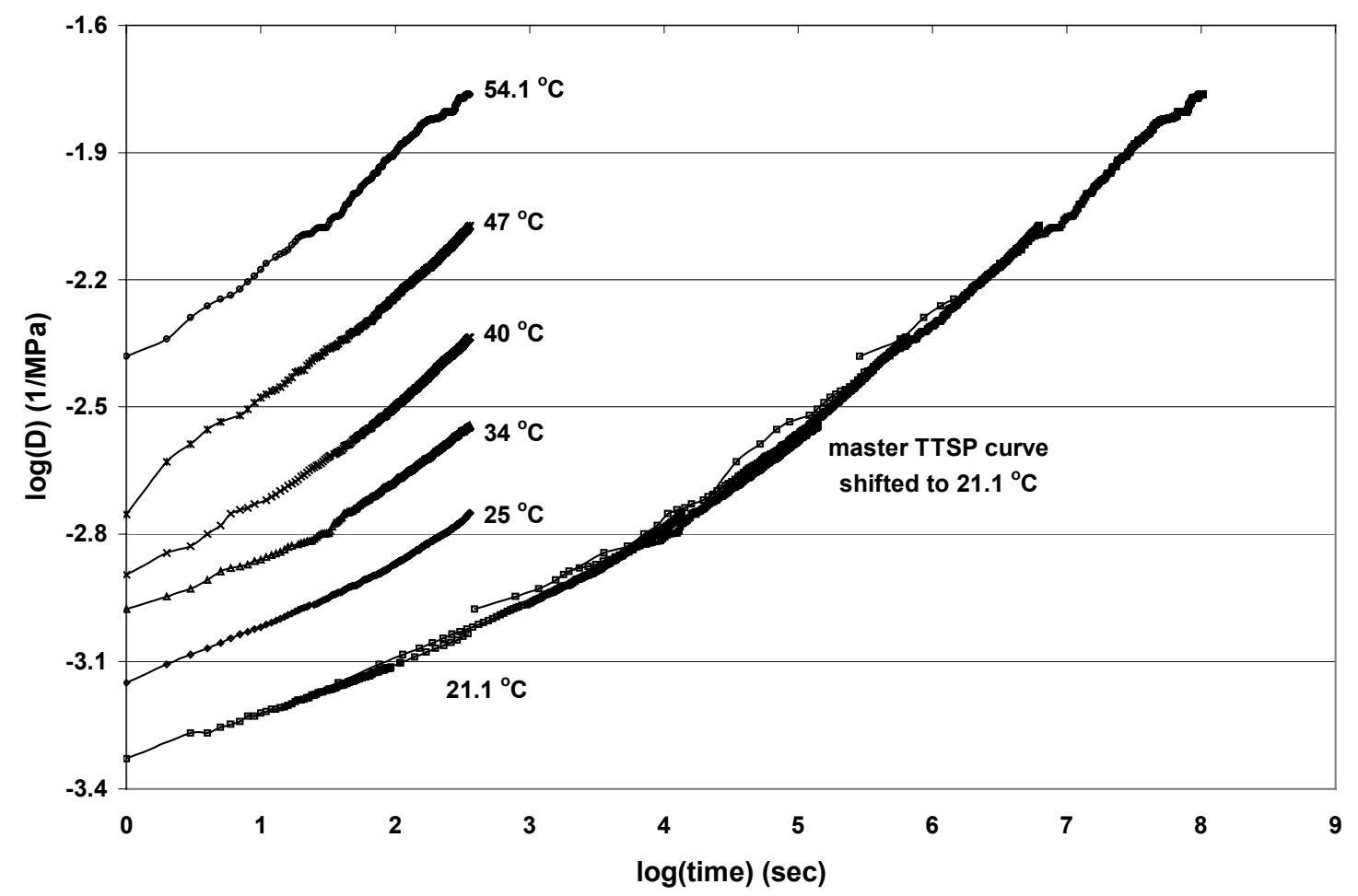

Figure 4.1. TTSP graphs and master curve shifted to $21.1^{\circ} \mathrm{C}$ of material A.

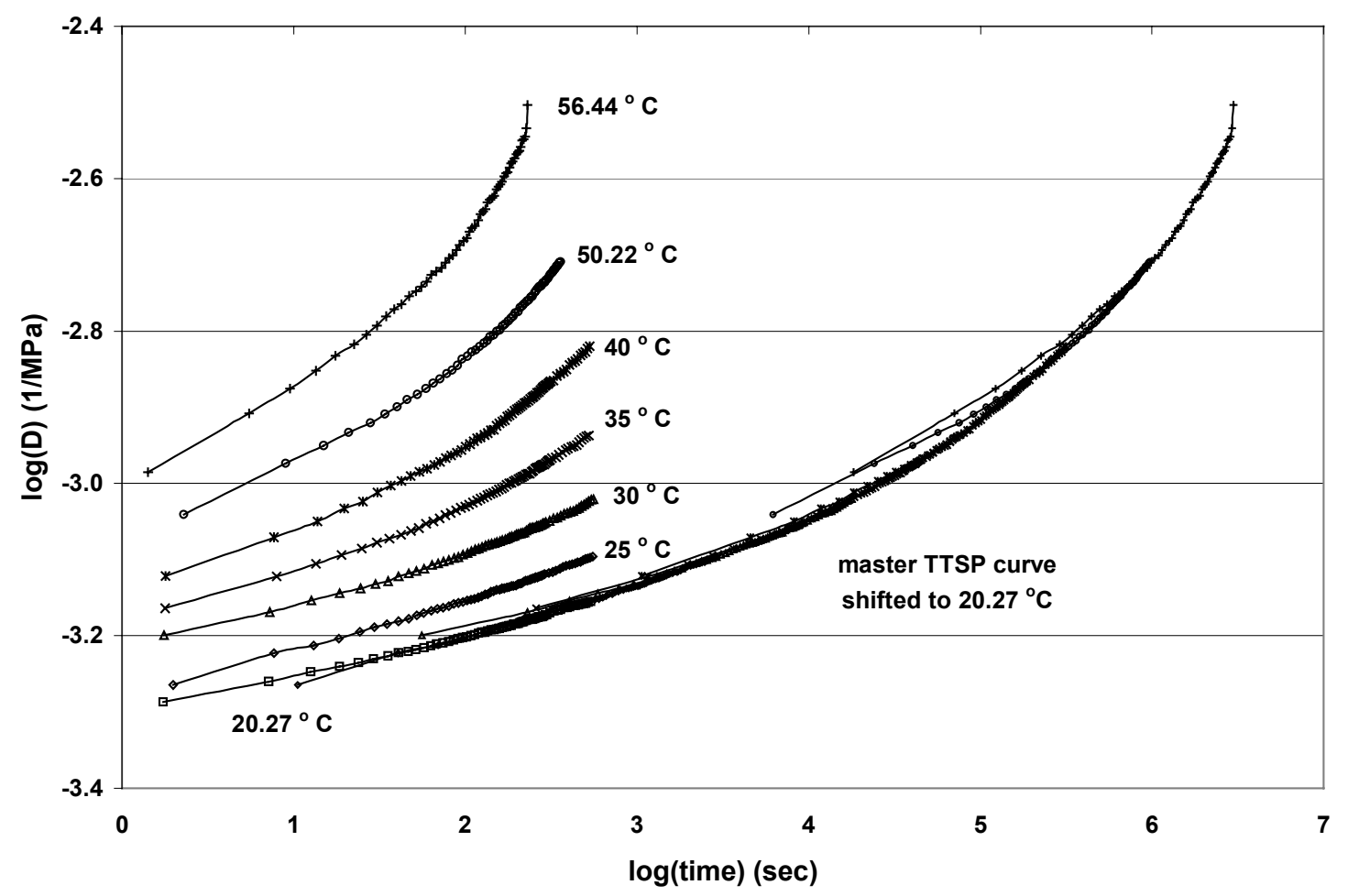

Figure 4.2. TTSP graphs and master curve shifted to $20.27^{\circ} \mathrm{C}$ of material B. 


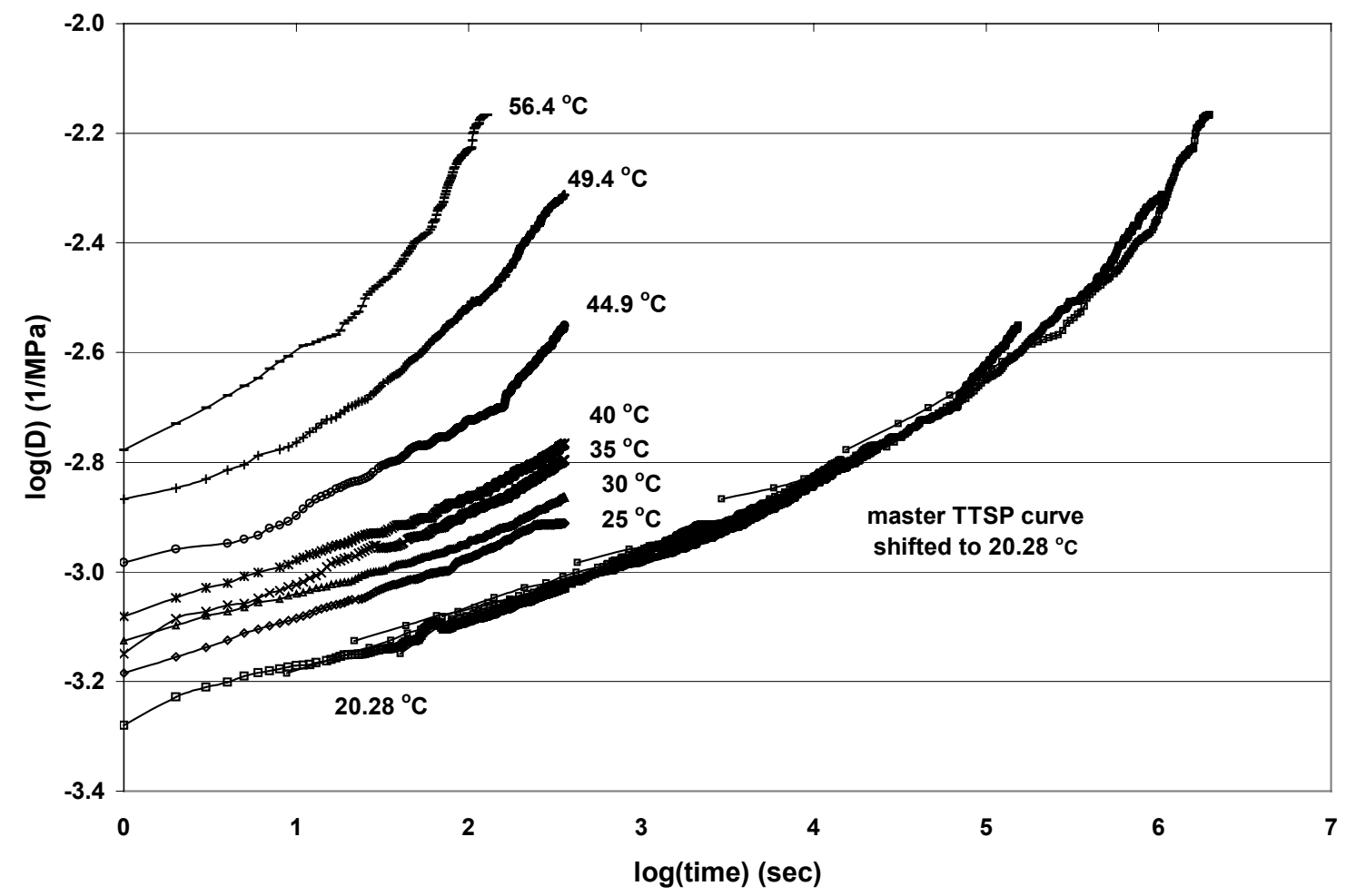

Figure 4.3. TTSP graphs and master curve shifted to $20.28{ }^{\circ} \mathrm{C}$ of material C.

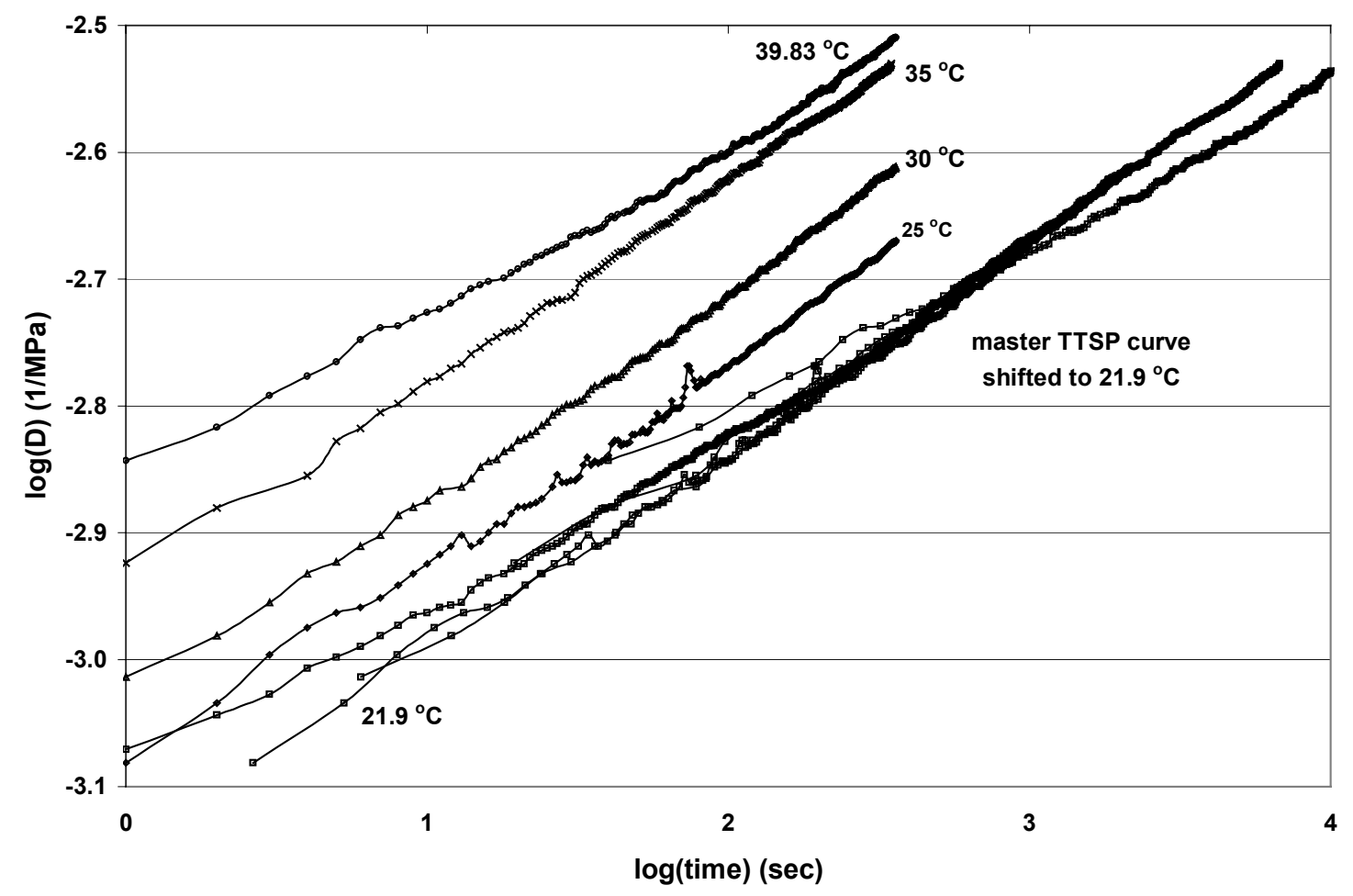

Figure 4.4. TTSP graphs and master curve shifted to $21.9^{\circ} \mathrm{C}$ of material D. 


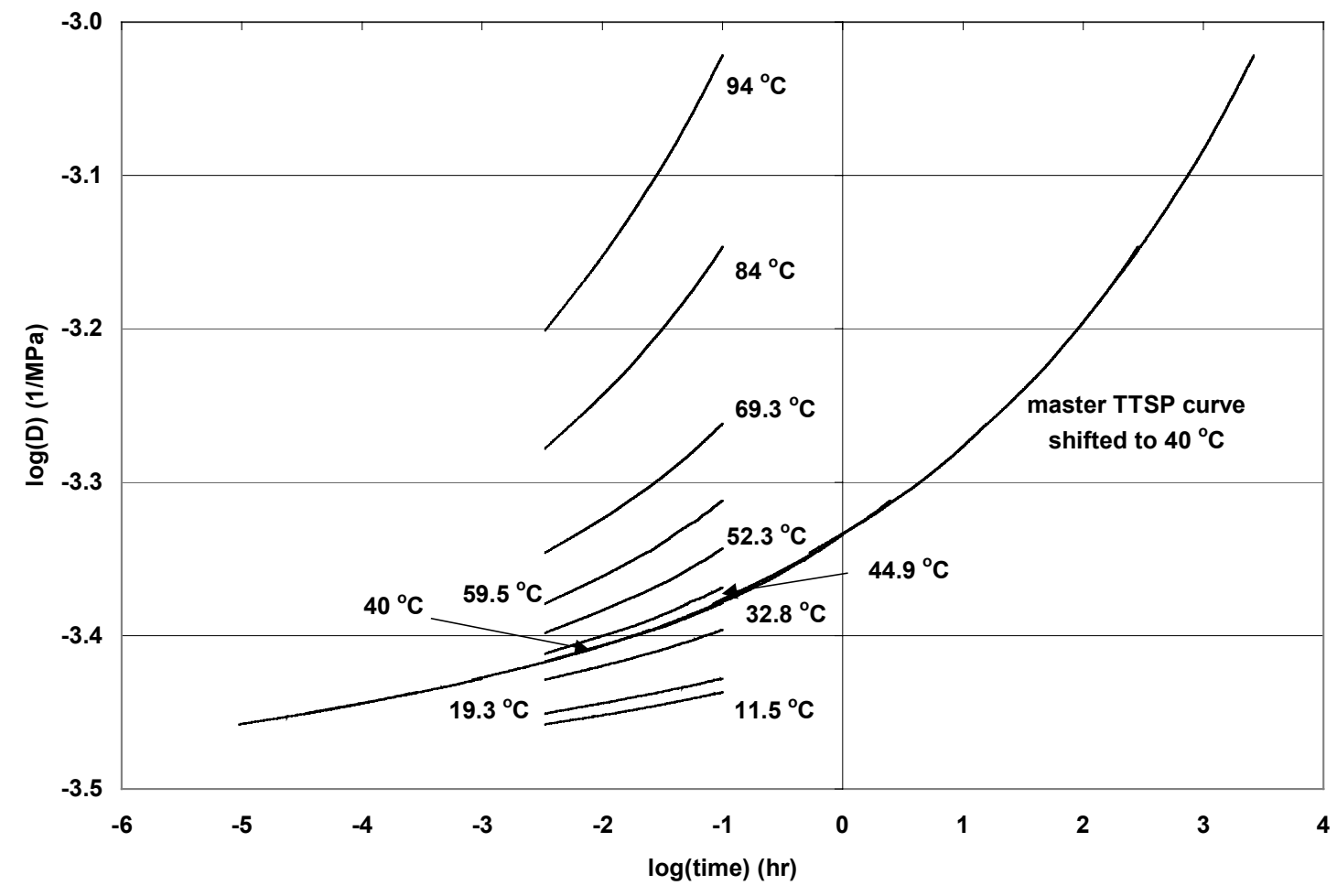

Figure 4.5. TTSP graphs and master curve shifted to $40{ }^{\circ} \mathrm{C}$ of material $\mathrm{E}$.

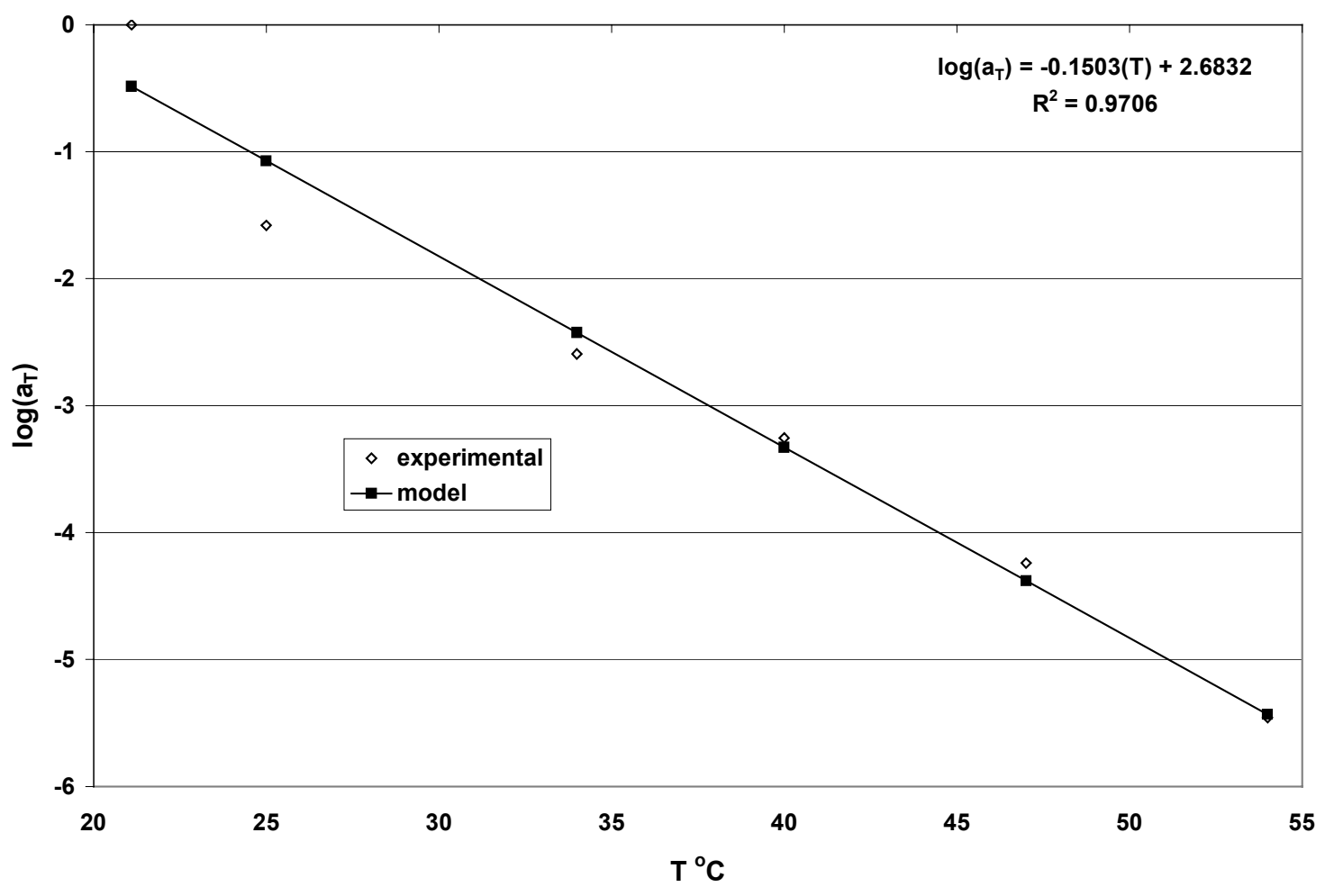

Figure 4.6. Theoretical and experimental values of the TTSP shift factors for material $\mathrm{A}$ at $21.1^{\circ} \mathrm{C}$. 


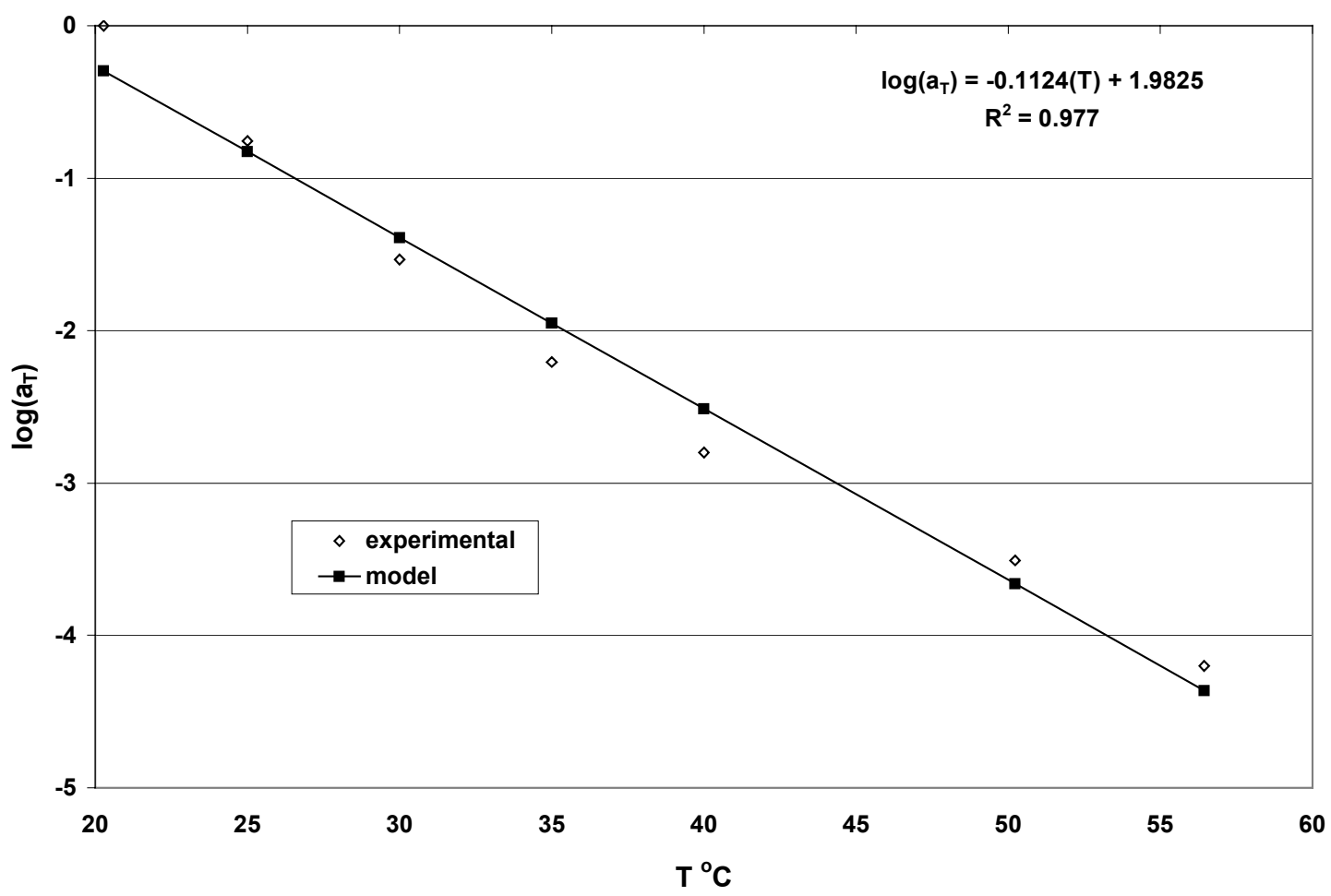

Figure 4.7. Theoretical and experimental values of the TTSP shift factors for material B at $20.27^{\circ} \mathrm{C}$.

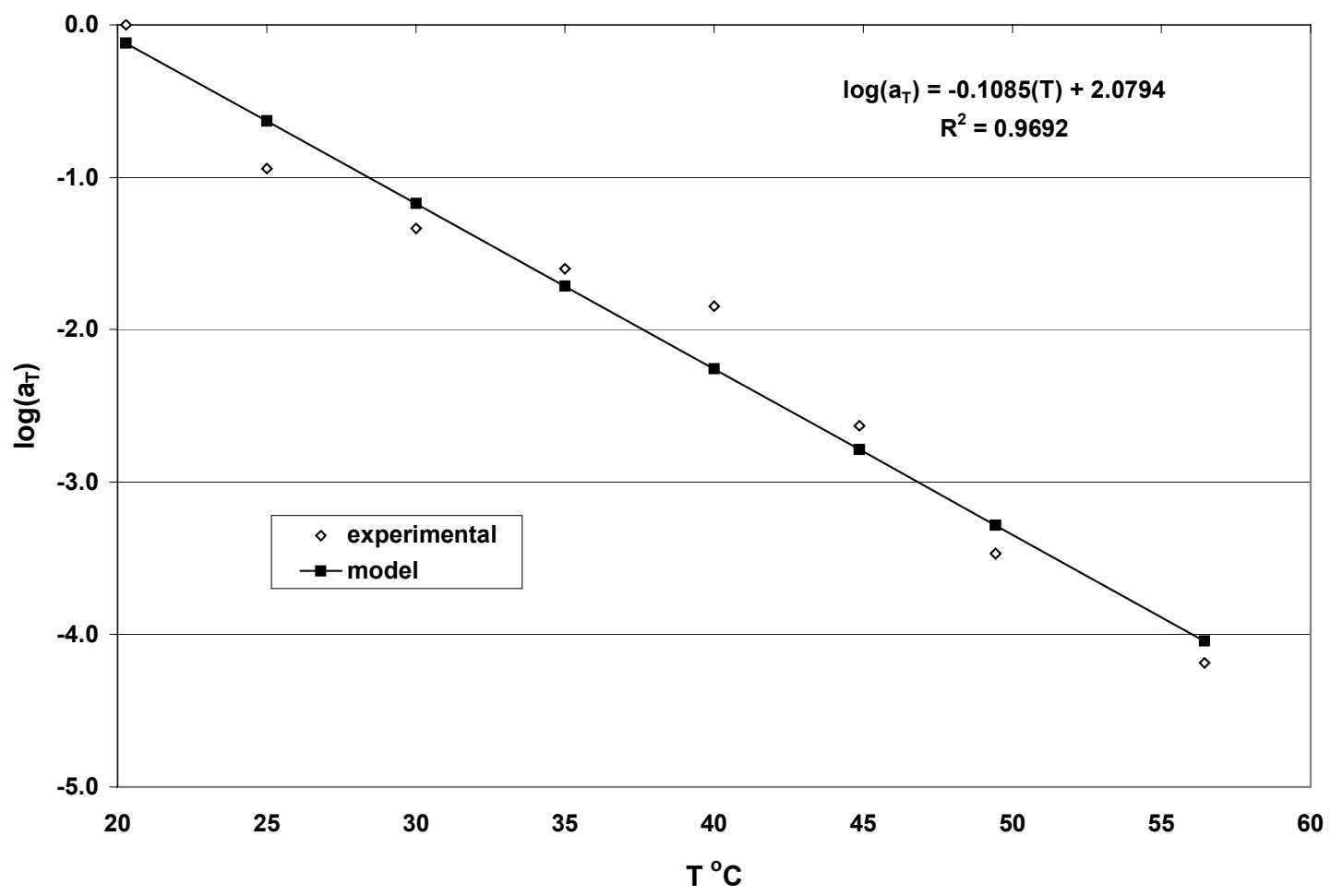

Figure 4.8. Theoretical and experimental values of the TTSP shift factors for material $\mathrm{C}$ at $20.28{ }^{\circ} \mathrm{C}$. 


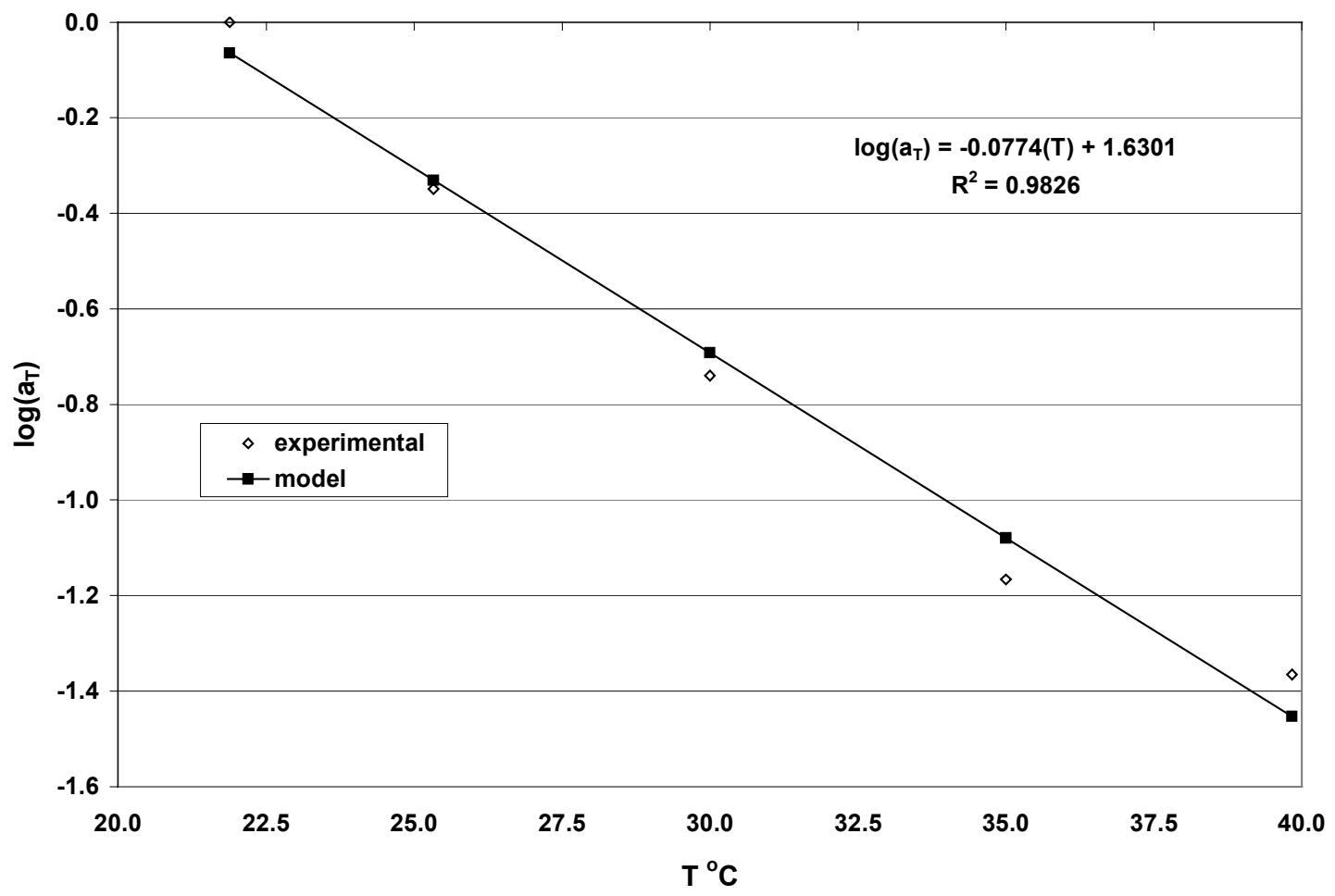

Figure 4.9. Theoretical and experimental values of the TTSP shift factors for material $D$ at $21.9{ }^{\circ} \mathrm{C}$.

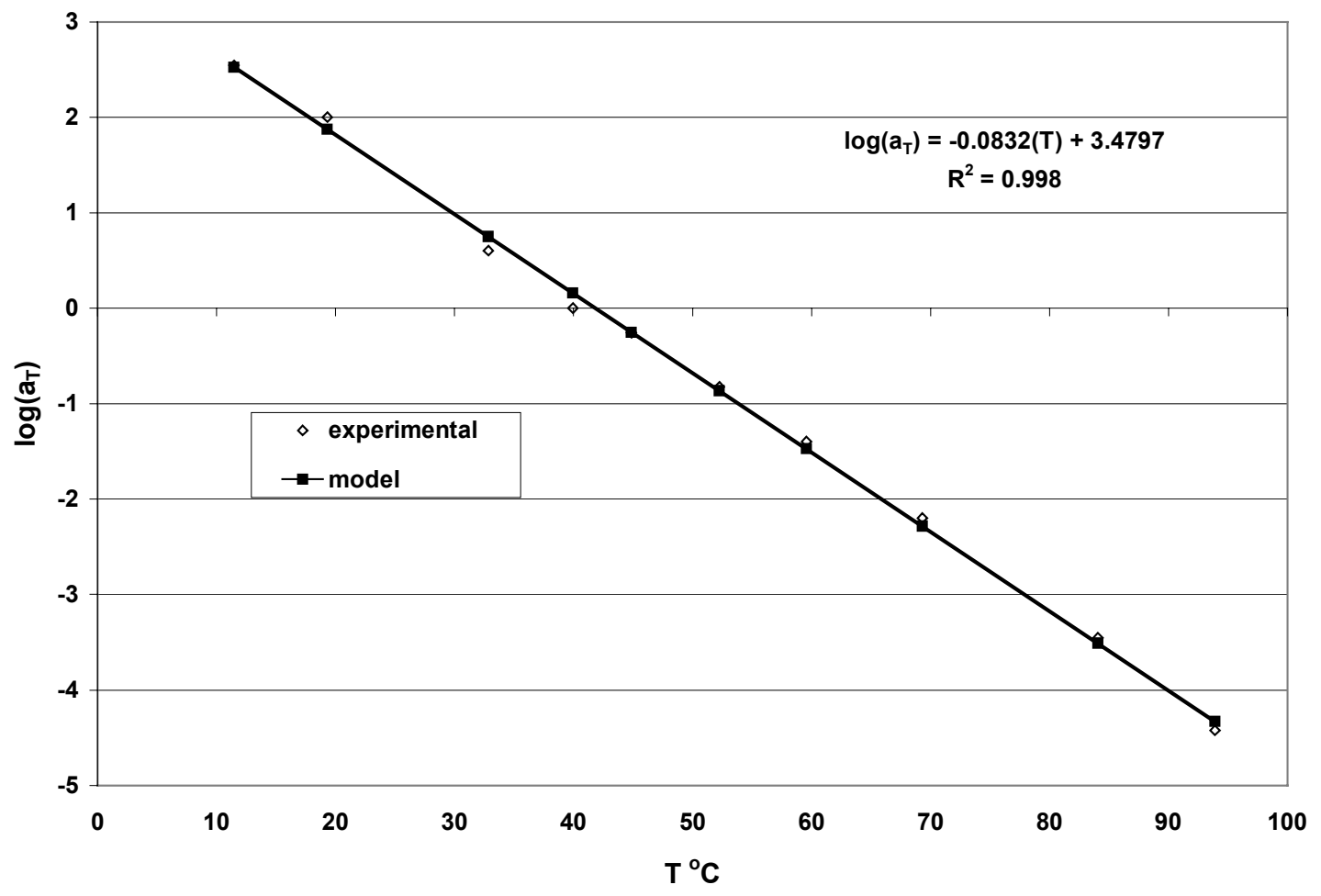

Figure 4.10. Theoretical and experimental values of the TTSP shift factors for material $\mathrm{E}$ at $40^{\circ} \mathrm{C}$. 


\section{CHAPTER 5 LONG-TERM TESTING AND CORRELATION WITH FULL SIZE SPECIMENS}

\subsection{Introduction}

The goal of this project was to be able to predict the ageing of the five materials and compare them with data from full-scale samples [20]. In order to compare the prediction and long-term tests with the full-scale samples, the data needed to be shifted for age and temperature outside the range of age and temperature used in the accelerated tests. In addition, sample size and testing conditions of full-size test are quite different from bending test. Long-term tests on the order of four or six days and a $t_{e}$ of $1 \mathrm{hr}$ were conducted using the LTB fixtures to give another set of data for comparison. The main advantage of doing long-term tests with the Long-Term Bending fixtures is that more of the test parameters could be controlled. This reduces the number of possible errors when trying to compare the results. By testing in this manner, we can tell if the predictions match long-term bending tests where both accelerated test and long-term bending tests correspond to samples of the same age albeit test durations are quite different. Performing LTB tests at two different temperatures also gives the opportunity to shift the creep compliance for temperature as well. Data taken in this study was shifted using the shift factors obtained from TTSP testing to $21.1{ }^{\circ} \mathrm{C}$. LTB data was then shifted to compensate for the difference in age and for temperature where needed. Data taken in this study was shifted using the shift factors obtained from TTSP testing Section 4.3 to $21.1{ }^{\circ} \mathrm{C}$. Table 5.1 is a list of the aging times, $\mathrm{t}_{\mathrm{e}}$, for each of the full-scale pipes. 


\subsection{Testing Procedure}

The testing procedure is similar to that of the ageing study and the TTSP. First, the specimens are annealed and then quenched. Next, they are aged one hour with two tests being preformed at $t_{e}=20 \min$ and $t_{e}=25$ min to mechanically condition the specimen. Finally, the test is started and data is recorded. Tests were performed at both $21.1{ }^{\circ} \mathrm{C}$ and $40{ }^{\circ} \mathrm{C}$ with a $\mathrm{t}_{\mathrm{e}}=1 \mathrm{hr}$. Data was recorded every 30 seconds and then a moving average was taken to reduce the number of points, making the data more manageable in Excel.

\subsection{Data Analysis}

\subsubsection{Shifting for Age and Temperature}

Struik [2] proposed that the equivalent time could be predicted using the following equation

$$
t=t_{e}\left[\left[\left[\left(\frac{\lambda \alpha}{t_{e}}\right)+1\right]^{\frac{1}{\alpha}}\right]-1\right]
$$

where $t$ is the equivalent time to compensate for ageing, $\lambda$ is the unaged time on the TTSP curve, $\alpha=1-\mu$, and $t_{e}$ is the age (time at which the LTB test was started). The compliance is then read on the TTSP curve and plotted against the times calculated using equation (5.1). Creep behaviors were predicted at both $21.1{ }^{\circ} \mathrm{C}$ and $40{ }^{\circ} \mathrm{C}$.

Next, the creep vs. time data taken at $21.1{ }^{\circ} \mathrm{C}$ was shifted to $40{ }^{\circ} \mathrm{C}$ and the data taken at $40{ }^{\circ} \mathrm{C}$ was shifted to $21.1{ }^{\circ} \mathrm{C}$. When shifting the data for temperature one must take into account that the stiffness of the material changes with temperature as well as with age. To shift data for temperature first calculate the unaged time, $\lambda$, using the following equation 


$$
\lambda=\left(\frac{t_{e}}{\alpha}\right)\left[\left(\frac{t}{t_{e}}+1\right)^{\alpha}-1\right]
$$

where $t$ is the test time, $t_{e}$ is the age of the material, and $\alpha=1-\mu$. The unaged time, $\lambda$, can be shifted for temperature and or age by dividing the unaged time by the corresponding shift factor $\left(\mathrm{a}_{\mathrm{T}}\right.$ to shift for temperature and or $\mathrm{a}_{\mathrm{e}}$ to shift for age) as shown in the following equation

$$
\lambda^{\prime}=\frac{\lambda}{a_{T}} \text { or } \frac{\lambda}{a_{e}} \text { or } \frac{\lambda}{a_{T} a_{e}}
$$

Once the unaged time is shifted the effective time or aged time can be calculated using the following equation

$$
t=t_{e}\left[\left[\left[\left(\frac{\lambda^{\prime} \alpha}{t_{e}}\right)+1\right]^{\frac{1}{\alpha}}\right]-1\right]
$$

The following flowchart illustrates the process of shifting the data for temperature and or age.

$$
\begin{aligned}
& \underset{t_{\text {real }}}{\mathrm{t}_{t}\left[(t)^{\alpha}\right]} \stackrel{\lambda}{\lambda} \quad \lambda^{\mid}=\frac{\lambda}{a_{T}} \operatorname{or} \frac{\lambda}{a_{e}} \operatorname{or} \frac{\lambda}{a_{T} a_{e}} \\
& \lambda=\frac{t_{e}}{\alpha}\left[\left(1+\frac{t}{t_{e}}\right)-1\right] \\
& t^{\mid}=\left[\left(\frac{\lambda^{\mid} \alpha}{t_{e}}+1\right)^{\frac{1}{\alpha}}-1\right] t_{e}
\end{aligned}
$$


Figs. 5.1 through 5.5 contain the data from this study shifted from $21.1{ }^{\circ} \mathrm{C}\left(\mathrm{t}_{\mathrm{e}}=1\right.$ hr) to $40{ }^{\circ} \mathrm{C}\left(\mathrm{t}_{\mathrm{e}}=1 \mathrm{hr}\right)$. Figs. 5.6 through 5.9 contain the data shifted from $40{ }^{\circ} \mathrm{C}\left(\mathrm{t}_{\mathrm{e}}=1\right.$ hr) to $21.1{ }^{\circ} \mathrm{C}\left(\mathrm{t}_{\mathrm{e}}=1 \mathrm{hr}\right)$. In order to compare the LTB data with the full-scale data the TTSP curve was shifted from an age of $1 \mathrm{hr}$ to the age of the pipes when testing began

and the equivalent times, prediction, were calculated using equation 5.1. Figs. 5.10 through 5.14 are the comparisons of the full-scale data and the prediction based on Struik's effective-time theory.

\subsubsection{Calculating Ageing Shift Factors}

Using the ageing parameters obtained from Section 3.3 and the linear regression of the $\log \left(\mathrm{a}_{\mathrm{T}}\right)$ vs. $\log \left(\mathrm{t}_{\mathrm{e}}\right)$, the ageing factor $\mathrm{a}_{\mathrm{e}}$ can be calculated as

$$
a_{e}=10^{\left(b+\mu \log \left(t_{e}\right)\right)}
$$

where $\mathrm{b}$ is the $\mathrm{y}$ intercept, $\mu$ is the ageing parameter, and $t_{\mathrm{e}}$ is the age of the material. Since any ageing time can be used as reference time, we can take the age of the material on the TTSP as a reference ( $1 \mathrm{hr}$ in this study). For the reference age $\mathrm{a}_{\mathrm{e}}=1$ so

$$
b=-\mu \log \left(t_{e}\right)
$$

\subsubsection{Calculating Temperature Shift Factors}

Linear regression of $\log \left(\mathrm{a}_{\mathrm{T}}\right)$ vs. temperature yields a linear equation that allows us to predict the shift factor $\mathrm{a}_{\mathrm{T}}(\mathrm{T})$ for any temperature. Using the linear regression equations obtained in Figs. 4.6 through 4.10 the temperature shift factor can be calculated using the following equation

$$
a_{T}=10^{(b+\gamma(T))}
$$


where $b$ is the $y$ intercept, $\gamma$ is the slope of the curve fit, and $T$ is the temperature. This method is similar to the method used to calculate $a_{e}$ described above. 
Table 5.1. Start date for testing of full-scale pipes.

\begin{tabular}{|c|c|c|c|c|}
\hline Material & Manufacture Date & Test Date & $\mathbf{T}_{\mathbf{e}}(\mathbf{h r})$ & $\mathbf{a}_{\mathbf{e}}$ \\
\hline$A$ & $10 / 11 / 2000$ & $12 / 04 / 2000$ & $1.30 \mathrm{E}+03$ & $3.19 \mathrm{E}-02$ \\
\hline$B$ & $02 / 01 / 2001$ & $05 / 02 / 2001$ & $2.16 \mathrm{E}+03$ & $3.96 \mathrm{E}-03$ \\
\hline$C$ & $02 / 01 / 2001$ & $05 / 02 / 2001$ & $2.16 \mathrm{E}+03$ & $4.05 \mathrm{E}-03$ \\
\hline$D$ & $12 / 07 / 2001$ & $04 / 03 / 2002$ & $2.81 \mathrm{E}+03$ & $2.46 \mathrm{E}-01$ \\
\hline$E$ & $11 / 29 / 2001$ & $04 / 01 / 2002$ & $2.95 \mathrm{E}+03$ & $8.16 \mathrm{E}-03$ \\
\hline
\end{tabular}

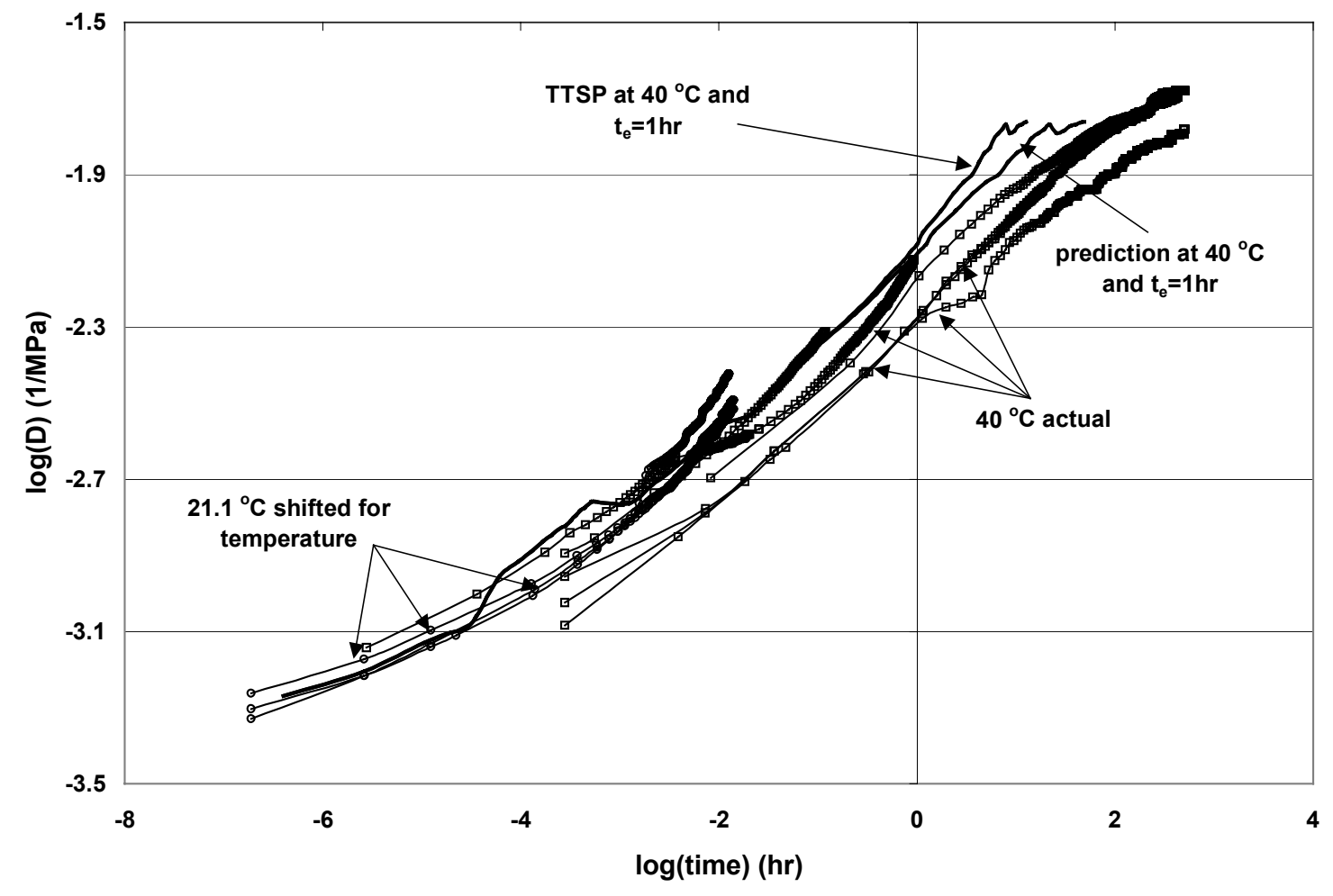

Figure 5.1. TTSP and prediction at $40^{\circ} \mathrm{C}$ and $t_{\mathrm{e}}=1 \mathrm{hr}$ for material A. 


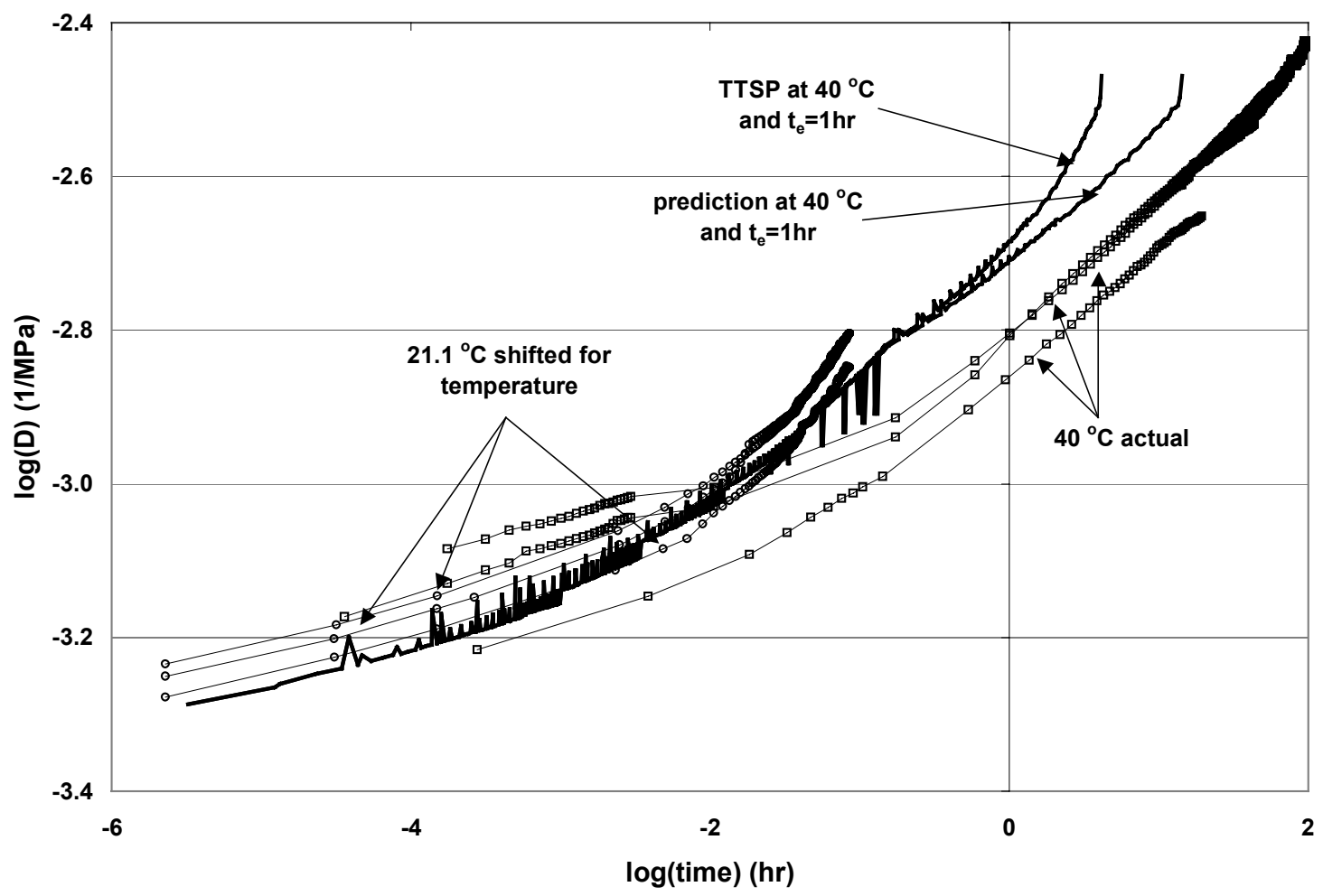

Figure 5.2. TTSP and prediction at $40{ }^{\circ} \mathrm{C}$ and $t_{\mathrm{e}}=1 \mathrm{hr}$ for material $\mathrm{B}$.

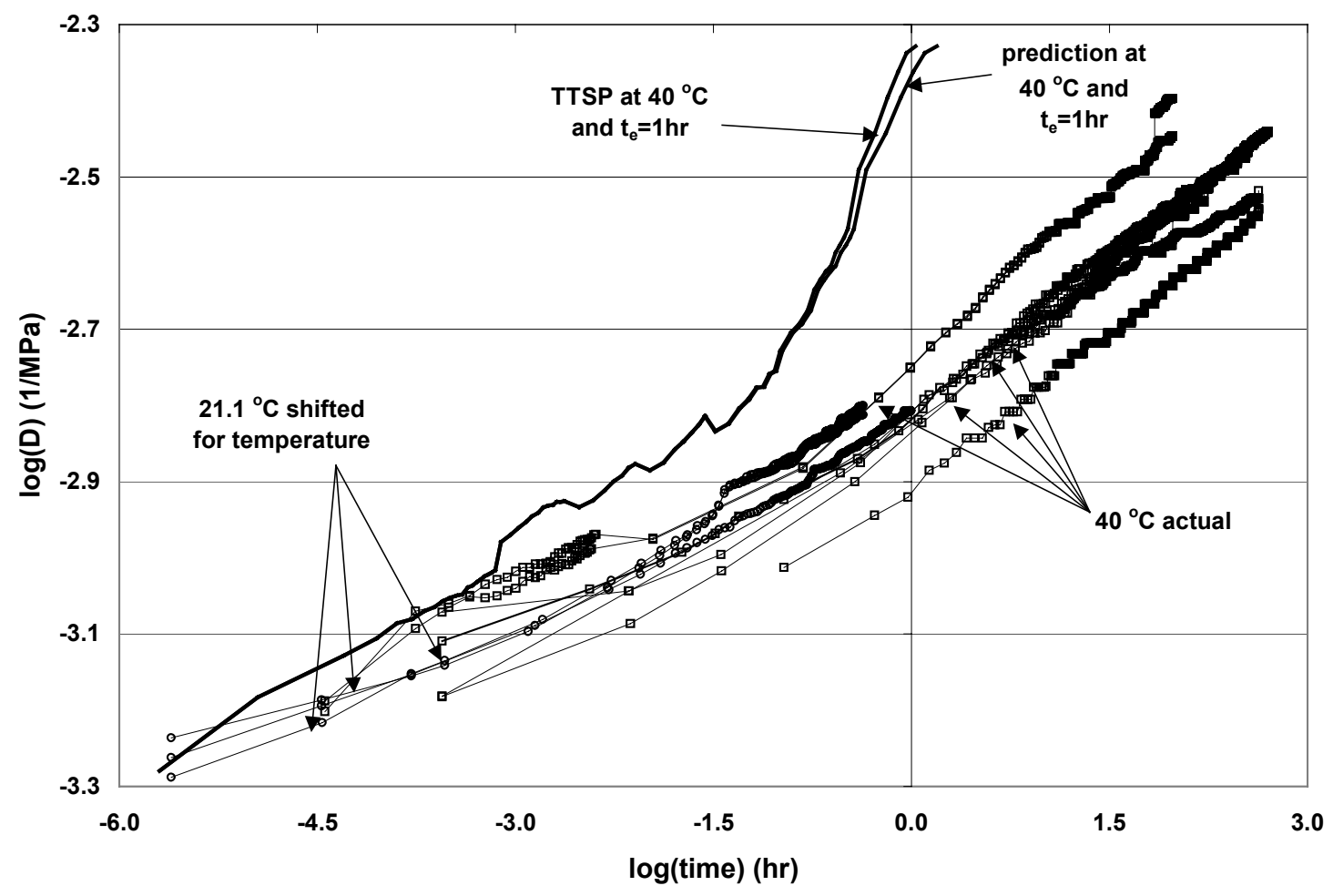

Figure 5.3. TTSP and prediction at $40^{\circ} \mathrm{C}$ and $t_{\mathrm{e}}=1 \mathrm{hr}$ for material $\mathrm{C}$. 


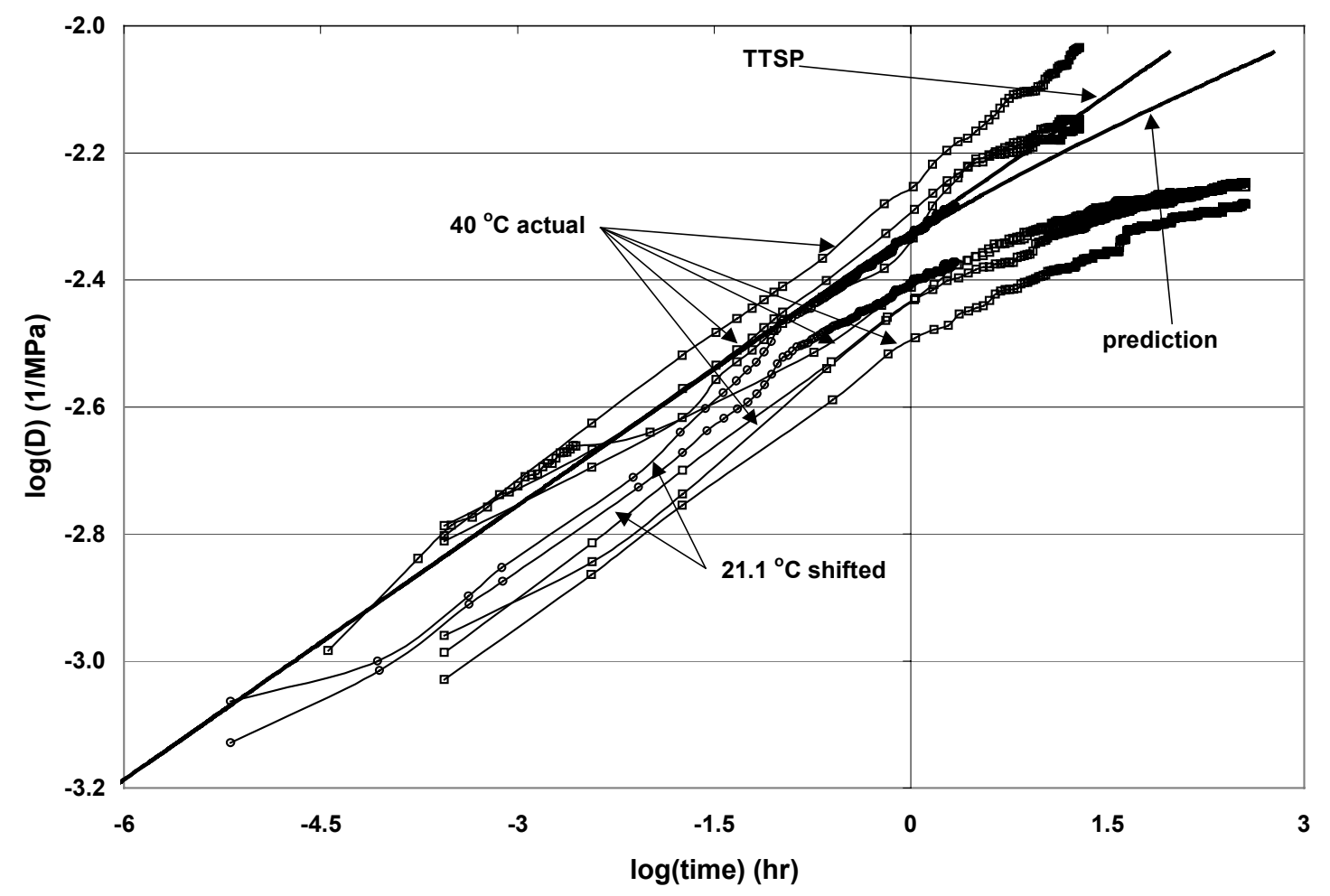

Figure 5.4. TTSP and prediction at $40^{\circ} \mathrm{C}$ and $t_{\mathrm{e}}=1 \mathrm{hr}$ for material $\mathrm{D}$.

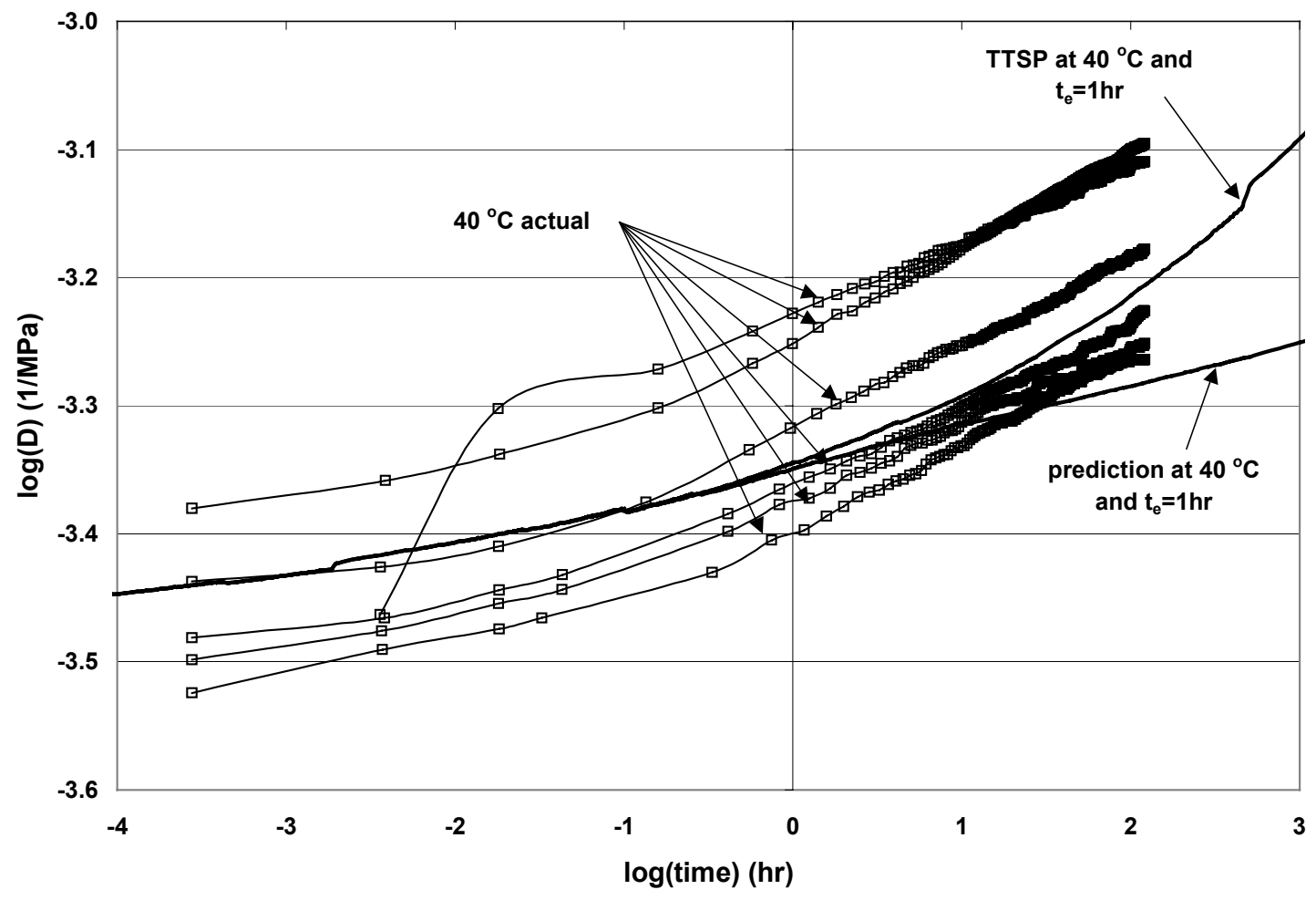

Figure 5.5. TTSP and prediction at $40{ }^{\circ} \mathrm{C}$ and $t_{e}=1 \mathrm{hr}$ for material $\mathrm{E}$. 


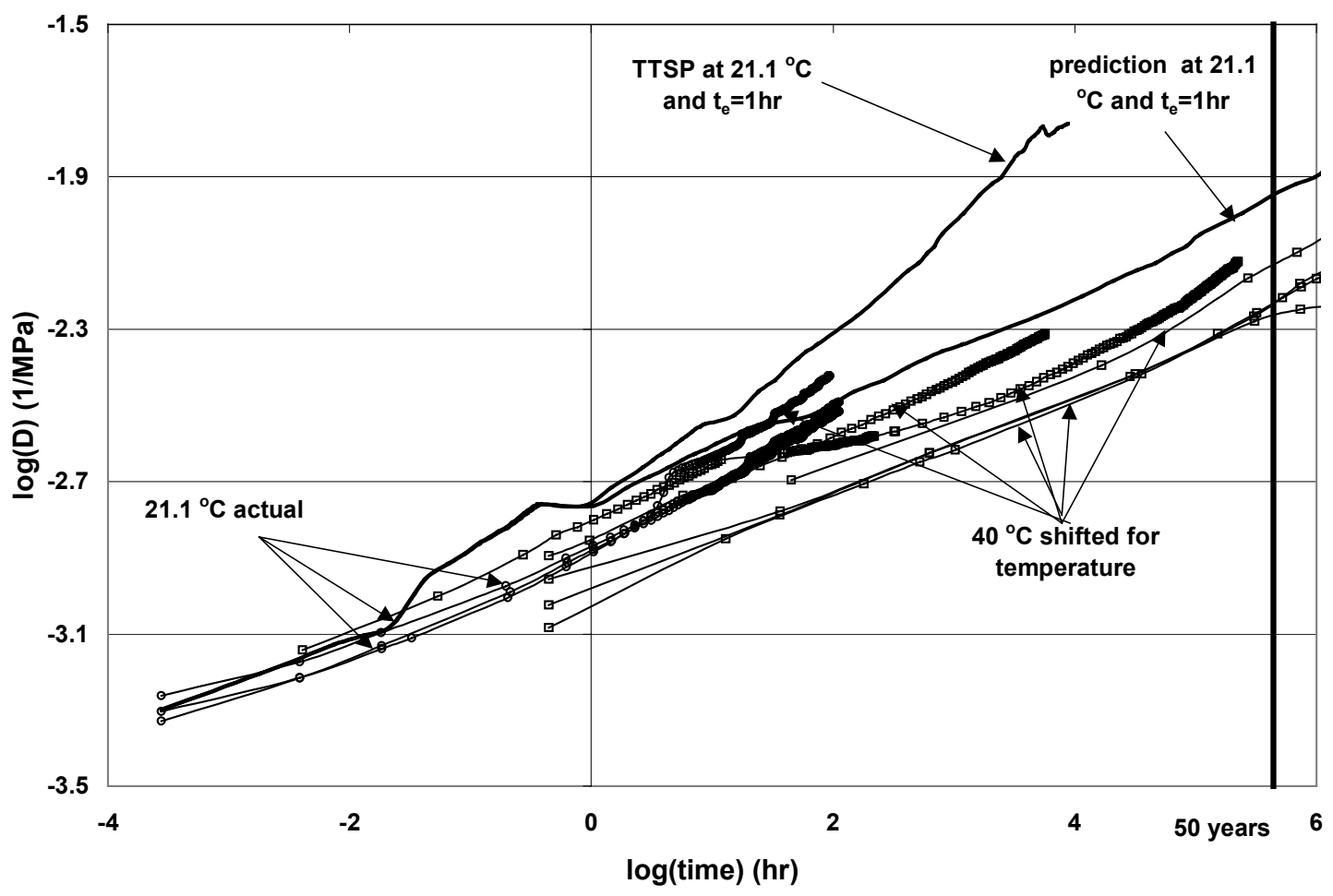

Figure 5.6. TTSP and prediction at $21.1{ }^{\circ} \mathrm{C}$ and $\mathrm{t}_{\mathrm{e}}=1 \mathrm{hr}$ for material $\mathrm{A}$.

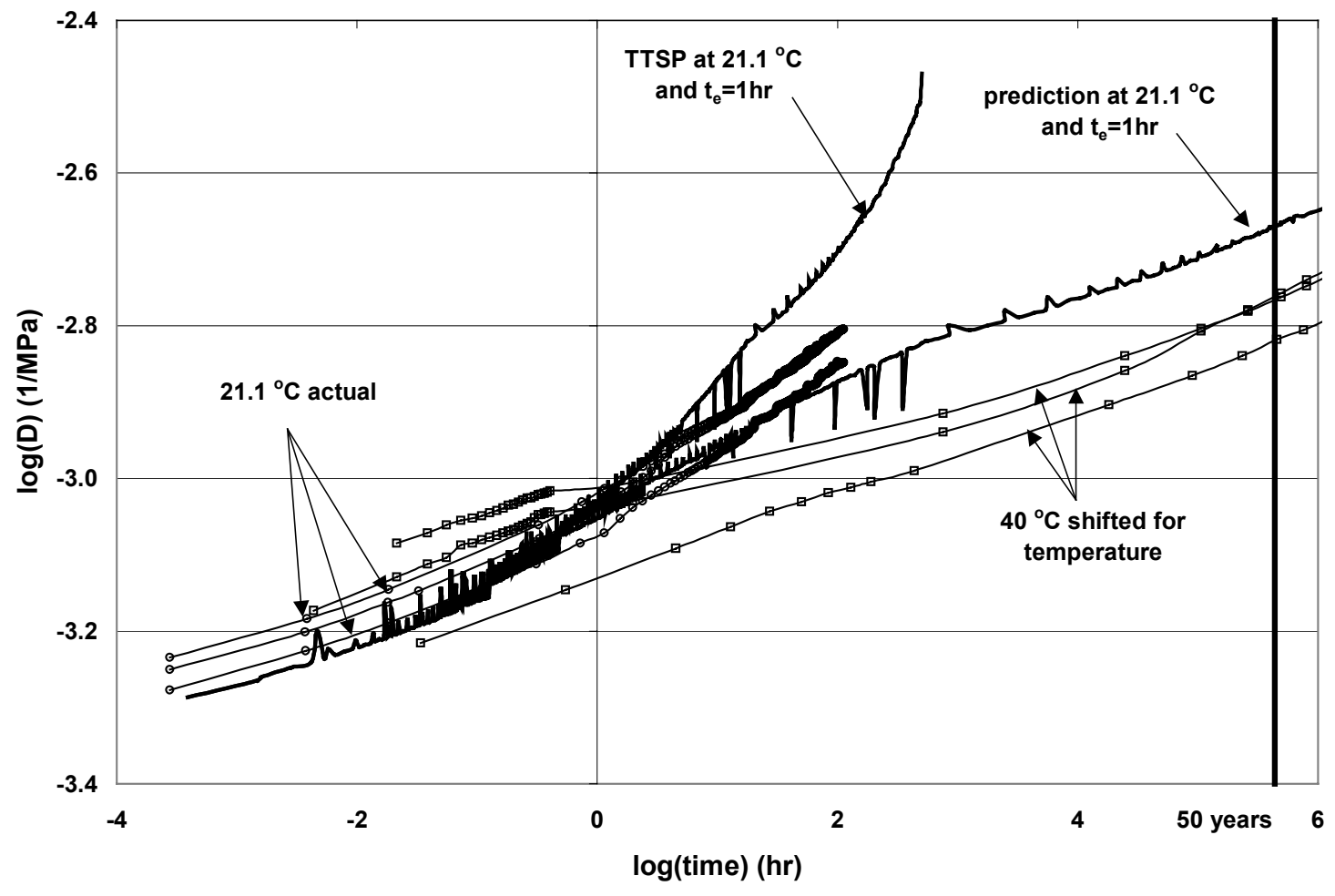

Figure 5.7. TTSP and prediction at $21.1^{\circ} \mathrm{C}$ and $t_{\mathrm{e}}=1 \mathrm{hr}$ for material $\mathrm{B}$. 


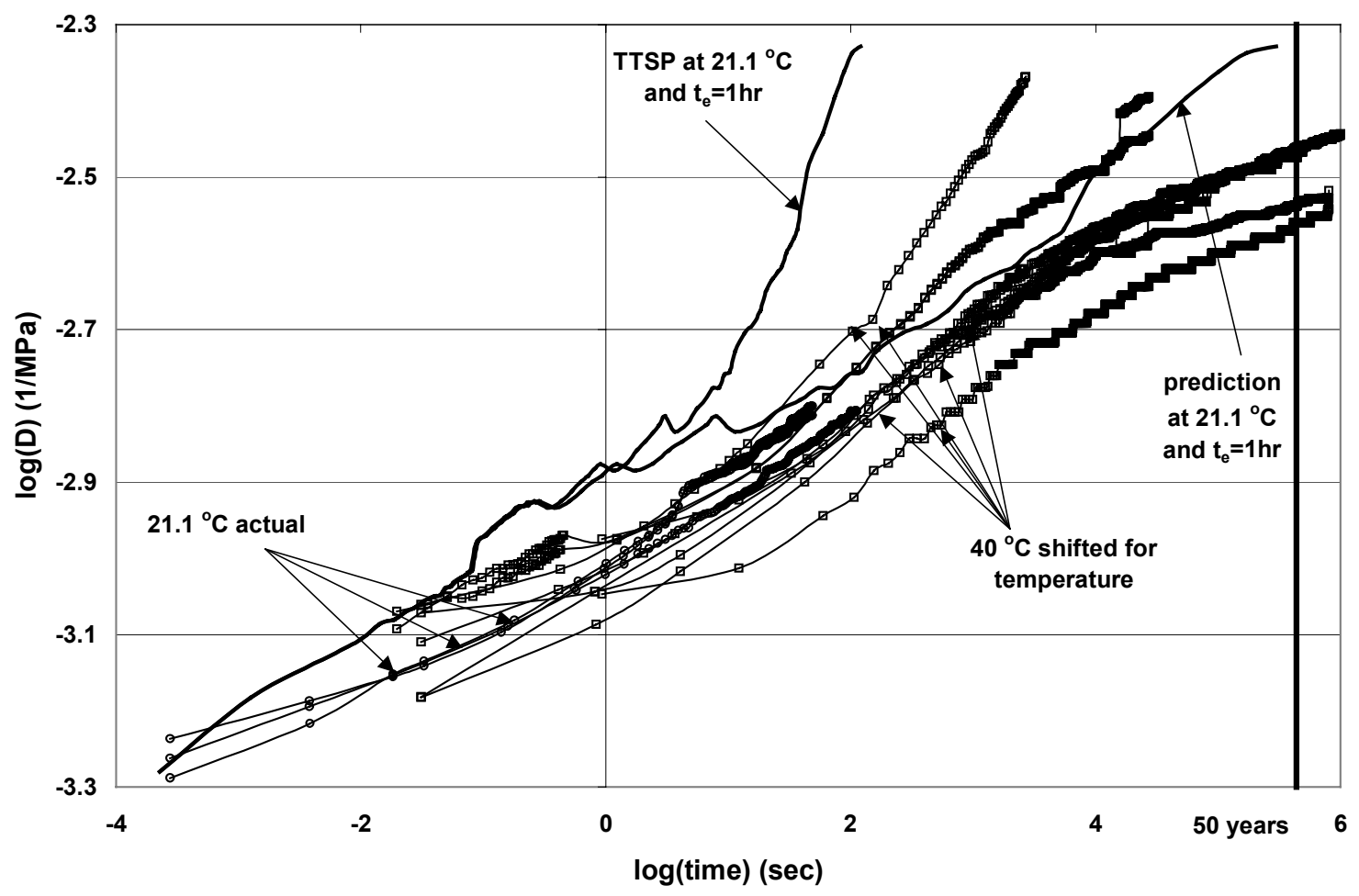

Figure 5.8. TTSP and prediction at $21.1^{\circ} \mathrm{C}$ and $\mathrm{t}_{\mathrm{e}}=1 \mathrm{hr}$ for material $\mathrm{C}$.

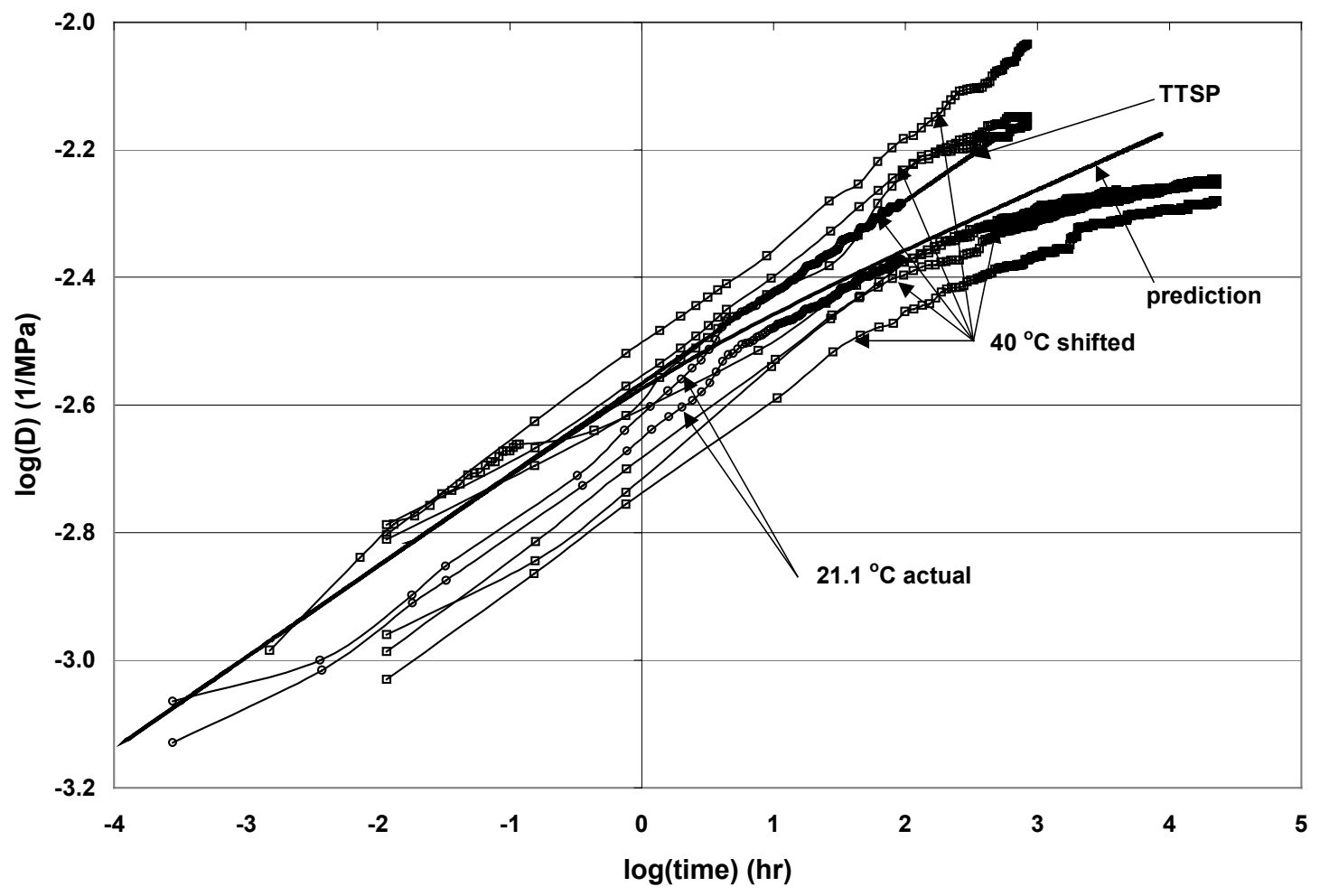

Figure 5.9. TTSP and prediction at $21.1^{\circ} \mathrm{C}$ and $t_{\mathrm{e}}=1 \mathrm{hr}$ for material $\mathrm{D}$. 


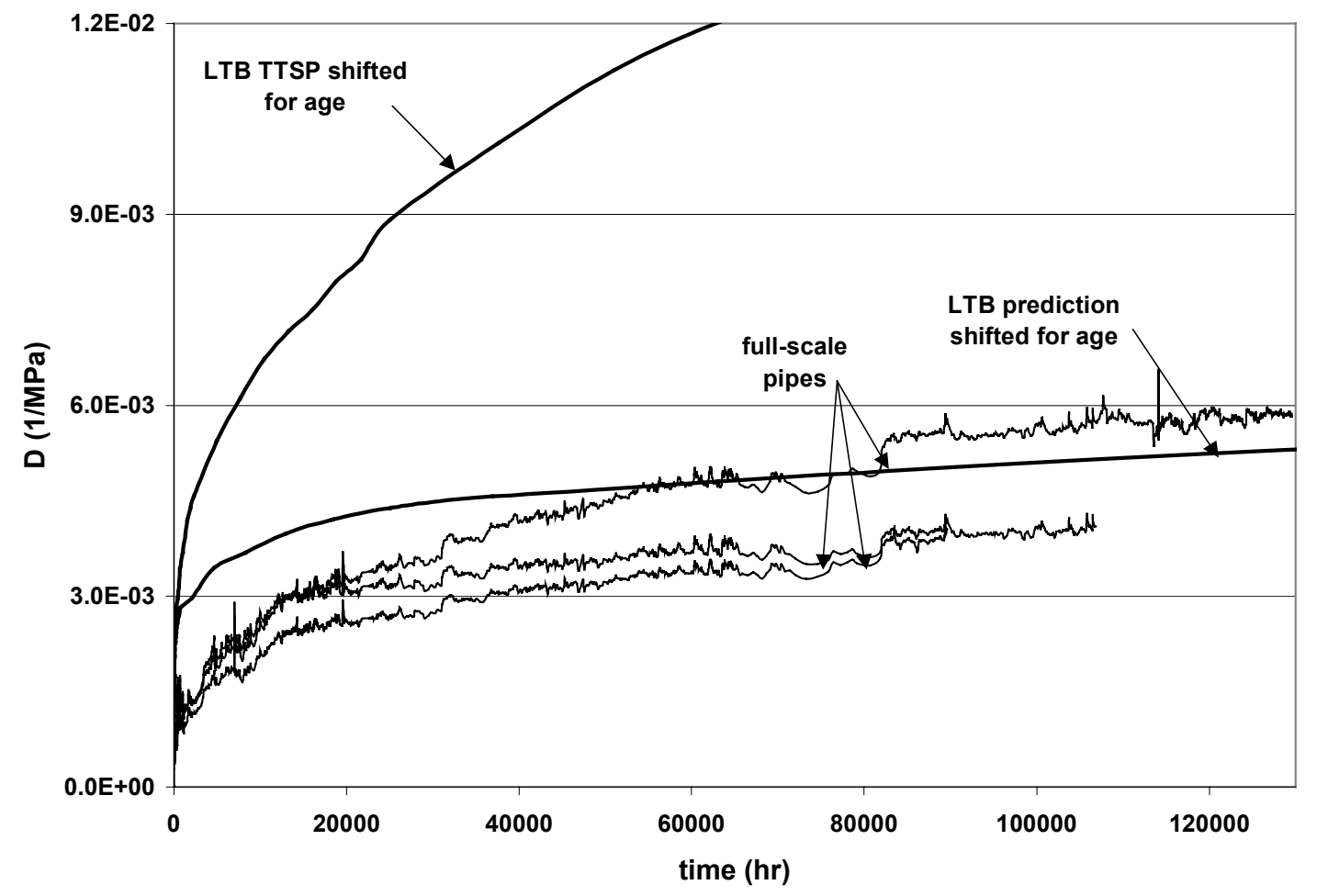

Figure 5.10. Comparison of full-size pipes and prediction for material A.

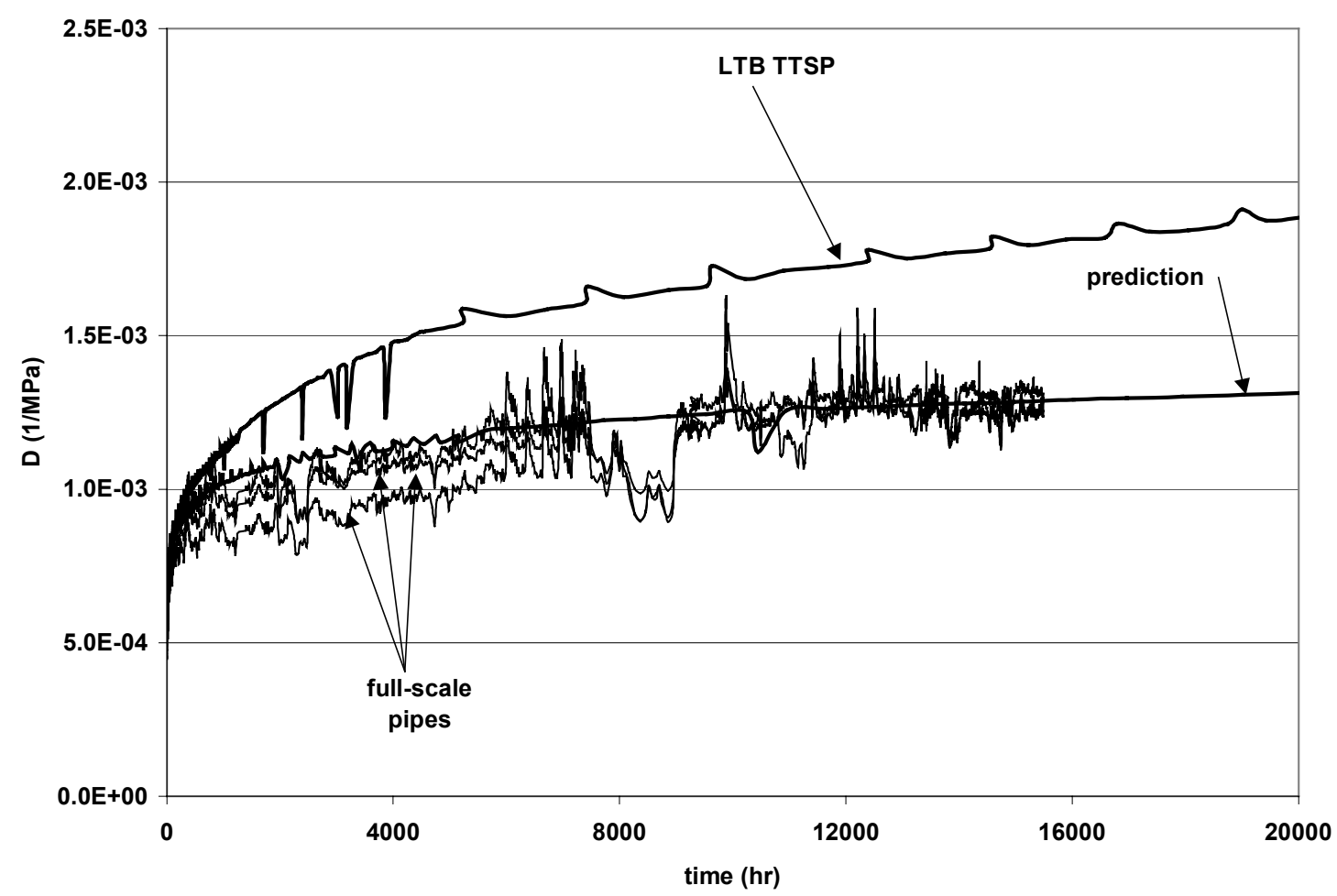

Figure 5.11. Comparison of full-size pipes and prediction for material B. 


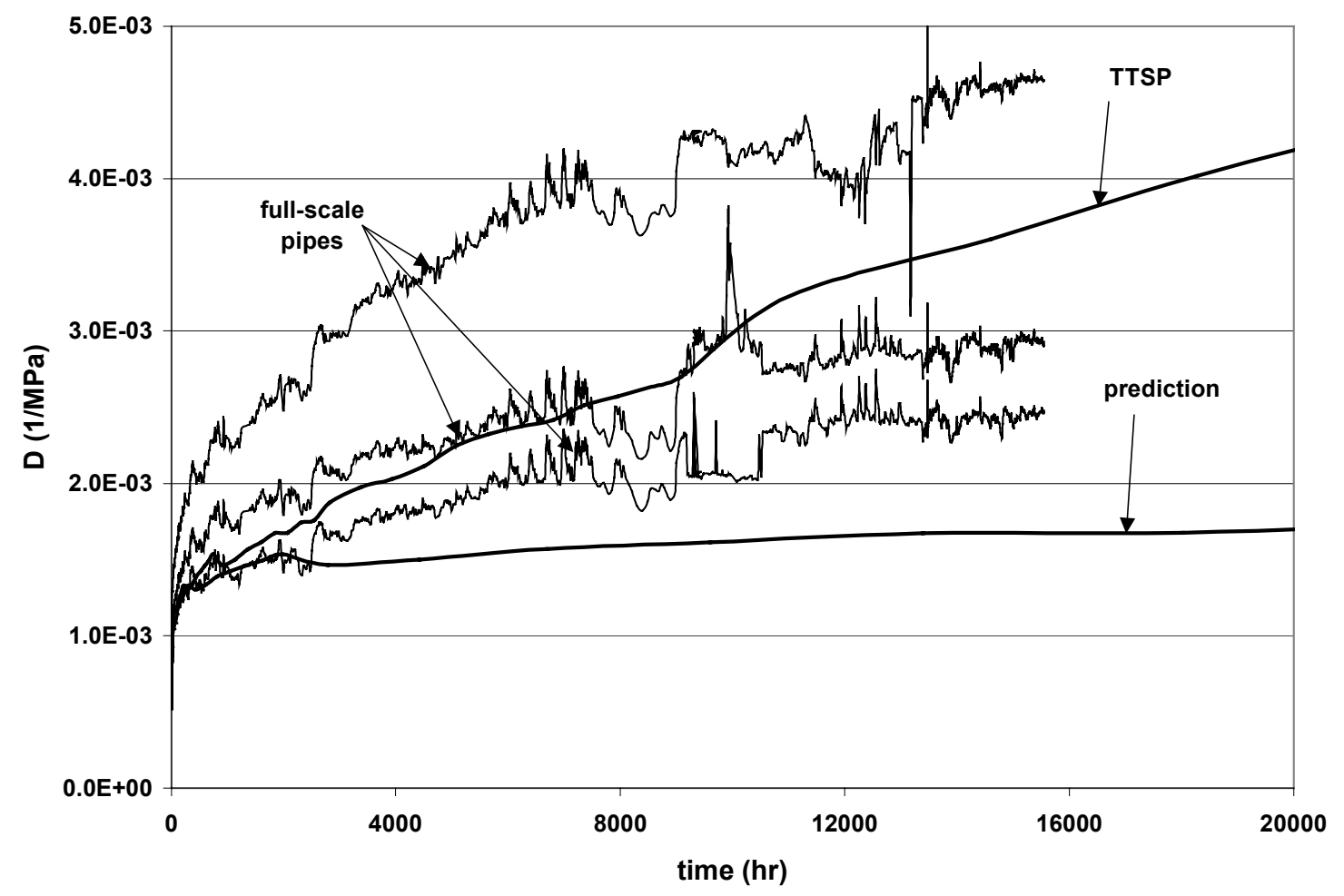

Figure 5.12. Comparison of full-size pipes and prediction for material $\mathrm{C}$.

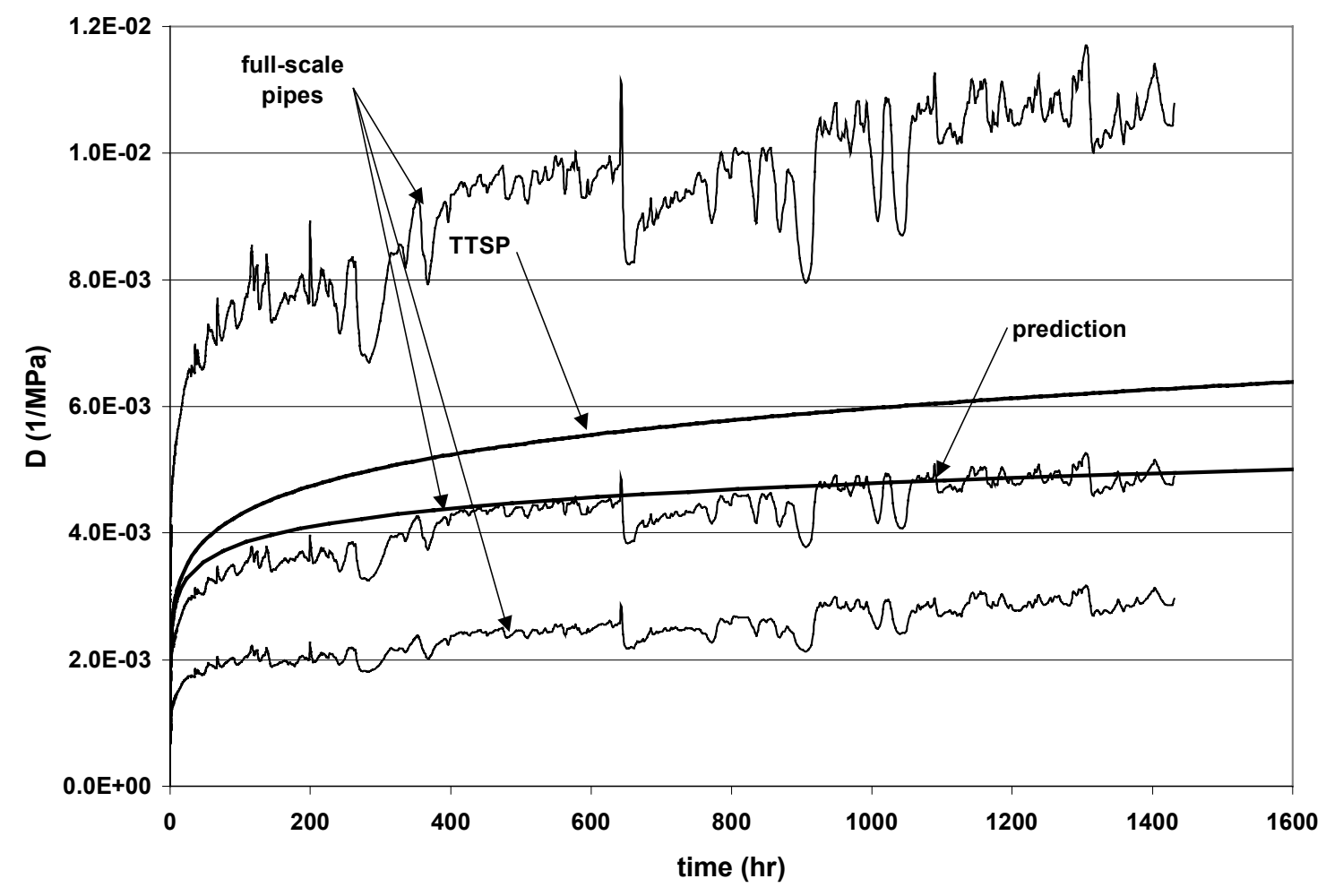

Figure 5.13. Comparison of full-size pipes and prediction for material D. 


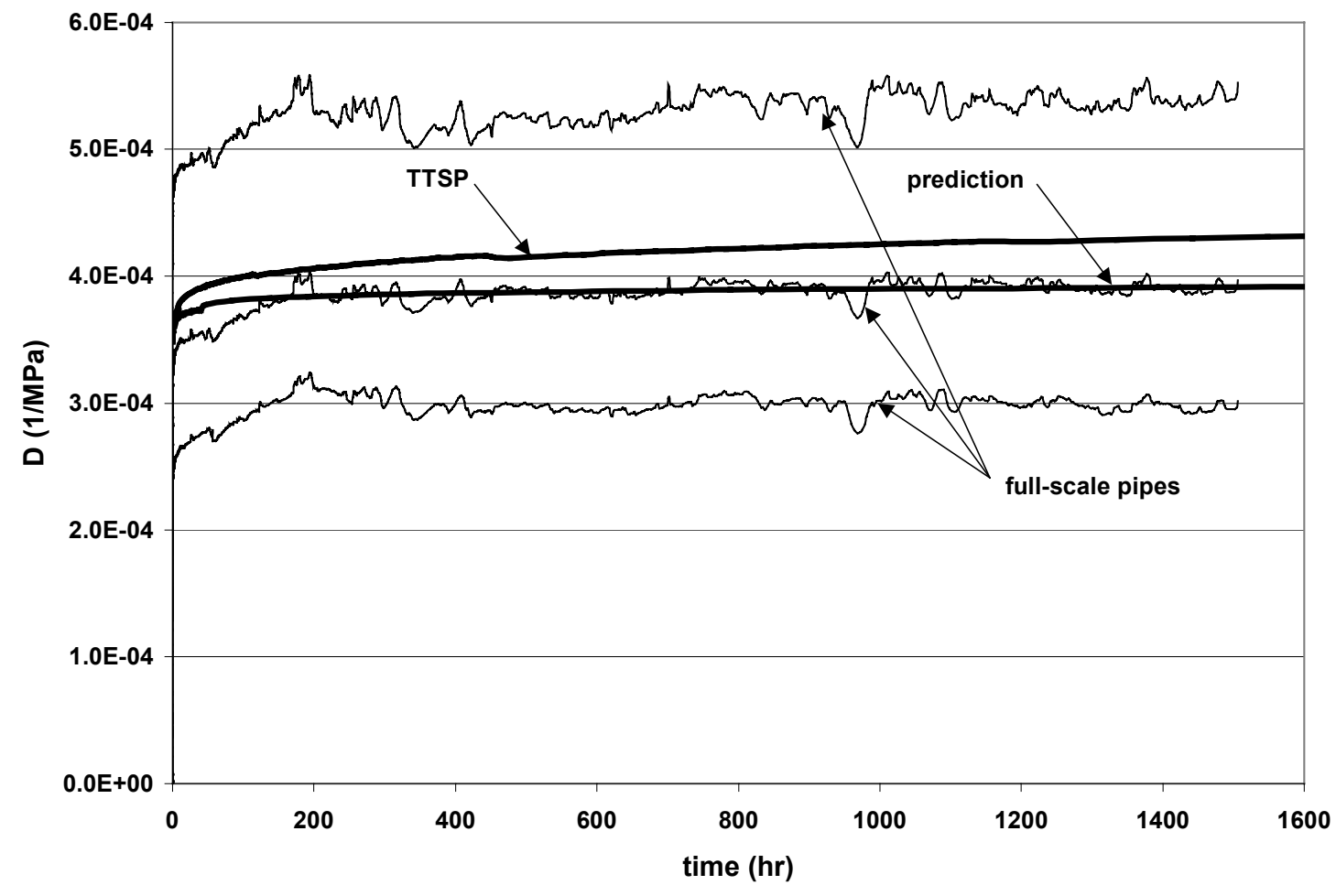

Figure 5.14. Comparison of full-size pipes and prediction for material $\mathbf{E}$. 


\section{CHAPTER 6 SUMMARY AND RECOMMENDATIONS}

\subsection{Summary}

This study was aimed at developing a short-term test that is an alternative to DMA testing to predict the time-dependent creep behaviors of commercial polymers. The proposed test procedure can be implemented regardless of the type of polymer material used for the liner. The liner materials used for testing are commercially available blends of polymers that are used in typical field installations.

It was determined that horizontal shifting alone is not enough to superimpose the creep curves obtained from an aging study as proposed by Struik [2]. A rotation is needed as proposed by Chai and McCrum [10] and Guerdoux et al. [15]. Through the fitting of the data with the Modified Power Law and then transforming the data into a straight line (Power Law) the data can be analytically superimposed. The aging parameter, $\mu$, is not affected by temperature, at least in the range of this study.

Shifting the data for temperature was performed quite well using the shift factors obtained from the TTSP curve. Struik's method for predicting the creep behavior correlated well with the experimental data from the LTB and full-size specimens. Materials A, B, D, and E followed the prediction for the time period tested. Material C however did not compare well with the prediction. It is believed that the full-size specimens yielded during testing, due to high strains.

\subsection{Contributions of this Thesis}

This thesis examined the physical aging behavior of five different materials: polyvinyl chloride, high-density polyethylene, and polyester reinforced with polyester fibers specimens. The objective of designing a short-term testing fixture capable of 
conducting the necessary experiments to predict the physical aging behavior of full-size specimens has been met using Struik's effective-time theory. It was shown that a horizontal shift alone is not enough to calculate the aging parameter of a material. A rotation as well as a horizontal shift of the data is needed. A method for shifting data to compensate for age and temperature was also presented.

\subsection{Recommendations}

The present study examined a method to predict the time-dependent creep behavior of five commercial products used in trenchless lining of pipes. The effectivetime theory and time temperature superposition principle were used for the basis of this

study. In addition to the above procedure, certain other areas of testing and theoretical analysis can be performed on materials to evaluate their structural behavior over time. Based on this study the following recommendations are made:

1. Investigate the sequential aging theory proposed by Struik to determine if the results are the same.

2. Study the effect of moisture on the long-term material properties of the liner.

3. Further analysis on the effect of annealing material D relative to its aging properties should be conducted.

4. More uniform specimens of material $\mathrm{E}$ should be obtained to improve the results of TTSP testing using the LTB.

5. Tests to determine the mechanical properties such as yield stress with a $t_{e}=1$ $\mathrm{hr}$ should be conducted. This would serve as a reference as to the maximum load to be applied during long-term testing. 


\section{REFERENCES CITED}

[1] Lakes, R. S., (1999) Viscoelastic Solids. CRC Press LLC, Boca Raton, Florida.

[2] Struik, L. C. E., (1978) Physical Aging in Amorphous Polymers and Other Materials. Elsevier Scientific Publishing Company, Amsterdam - Oxford - New York.

[3] Sharma, M. G., Viscoelasticity and Mechanical Properties of Polymers. Summer Institute on Applied Mechanics and Materials Science June 20-25, 1965, The Pennsylvania State University Department of Engineering Mechanics University Park, Pennsylvania.

[4] Wang, J. Z., Parvatareddy, H., Chang, T., Iyengar, N., Dillard, D. A., and Reifsnider, K. L. (1995) "Physical Aging Behavior of High-Performance Composites.” Composites Science and Technology. vol. 54, pp. 405-415.

[5] Knox, E. M., Cowling, M. J., and Hashim, S. A. (2000) "Creep analysis of adhesively bonded connections in GRE pipes including the effect of defects." Composites. Part A 31, pp. 583-590.

[6] Findley WN. "Combined stress creep of non-linear viscoelastic material." Smith AI, Nicolson AM, editors. Advances in creep design, London: Applied Science Publication, 1971 chap. 14.

[7] Bradshaw, R. D., and Brinson, L. C., (1997) “A Sign Control Method for Fitting and Interconverting Material Functions for Linearly Viscoelastic Solids.” Department of Mechanical Engineering Northwester University.

[8] Beckmann, J., McKenna, G. B., Landes, B. G., Bank, D. H., and Bubeck, R. A., (1997) "Physical Aging Kinetics of Syndiotactic Polystyrene as Determined From Creep Behavior." Polymer Engineering and Science. Vol. 37, No. 9 pp. 1459-1468. 
[9] Sullivan, J. L., (1990) "Creep and Physical Aging of Composites." Composite Science and Technology. Vol. 39, pp. 207-232.

[10] Chai, C. K., McCrum, N. G., (1980) "Mechanism of physical aging in crystalline polymers.” Polymer. Vol. 21 pp. 706-712.

[11] McCrum, N. G., (1992) “The interpretation of physical ageing in creep and DMTA from Sequential Ageing Theory." Plastics, Rubber and Composites Processing and Applications. Vol. 18, No. 3 pp. 181-191.

[12] Menard, K. P., (1999) Dynamical Mechanical Analysis. CRC Press LLC, Boca Raton, Florida.

[13] Kovacs, A. J., Strattton, R. A., Ferry, J. D., (1963) J. Phys. Chem., 67152.

[14] McCrum, N. G., (1984) Polymer Community, 252.

[15] Guerdoux, L., Duckett, R. A., Froelich, D., (1984) "Physical ageing of polycarbonate and PMMA by dynamic mechanical measurements." Polymer. Vol. 25 pp. 1392-1396.

[16] Dean, G. D., Read, B. E., Tomlins, P. E., (1990) "A model for long-term crep and the effects of physical aging in poly(butylenes terephthalate)." Plastics and rubber processing and applications. Vol. 13 NO 1 pp. 37-46.

[17] Barbero E. J., Julius M., and Yao Z., (2003) “Time and Frequency Viscoelastic Behavior of Commercial Polymers.” CCC-2003, Calabria, Italy.

[18] Barbero E. J., (2002) Finite Element Analysis of Composite Materials, in preparation.

[19] Beer, F. P., Johnston, E. R. Jr., (1992) Mechanics of Materials. McGraw-Hill, Inc. second edition. 
[20] Barbero E. J. and Rangarajan S., (2002) "Long-Term Testing of Trenchless Pipe Liners.” ASTM IPC-2002, Calgary, Alberta, Canada. 


\section{APPENDICES}

Appendix A - Drawing Package of Testing Apparatus

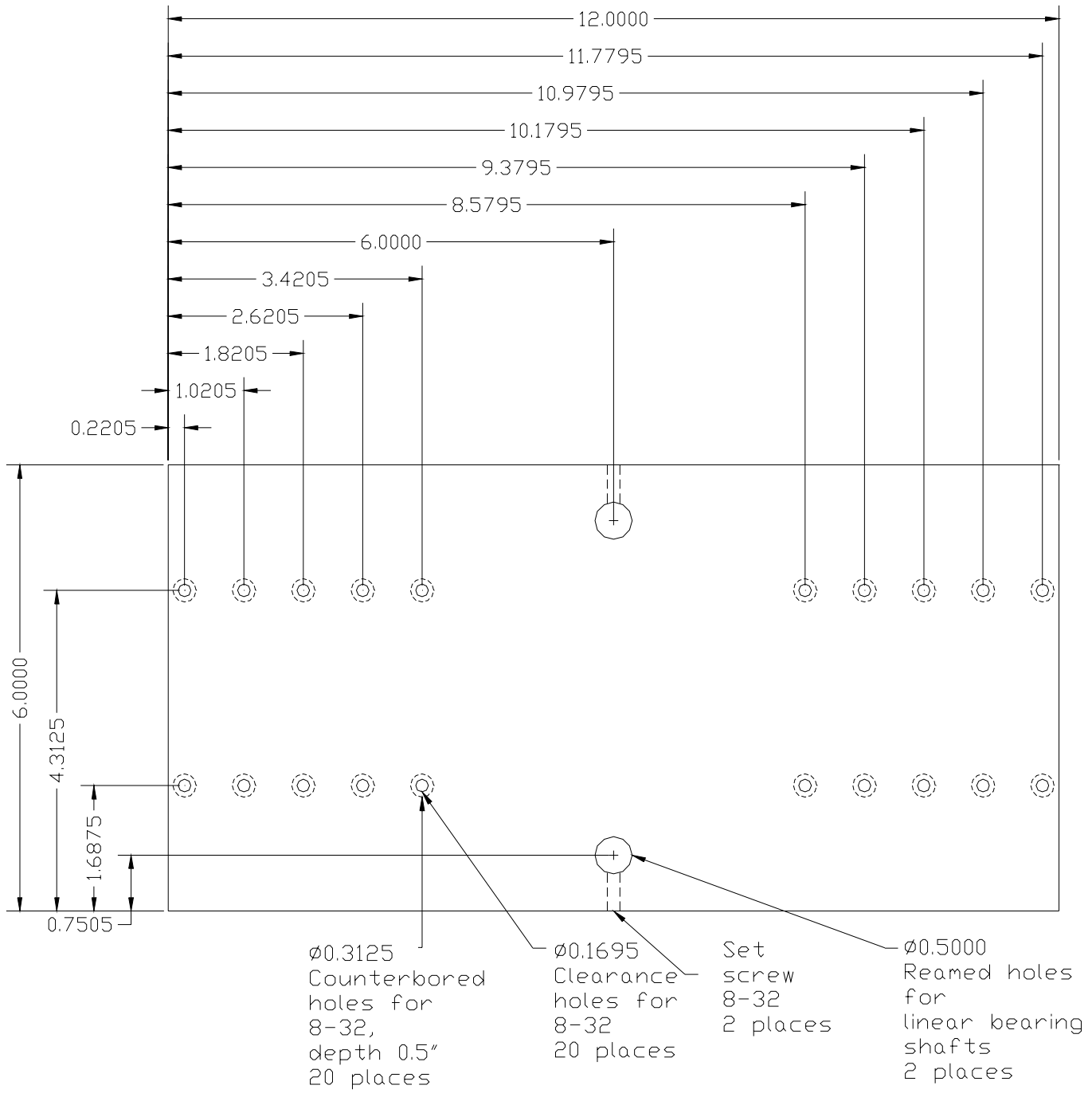

Figure A1 Testing fixture base 


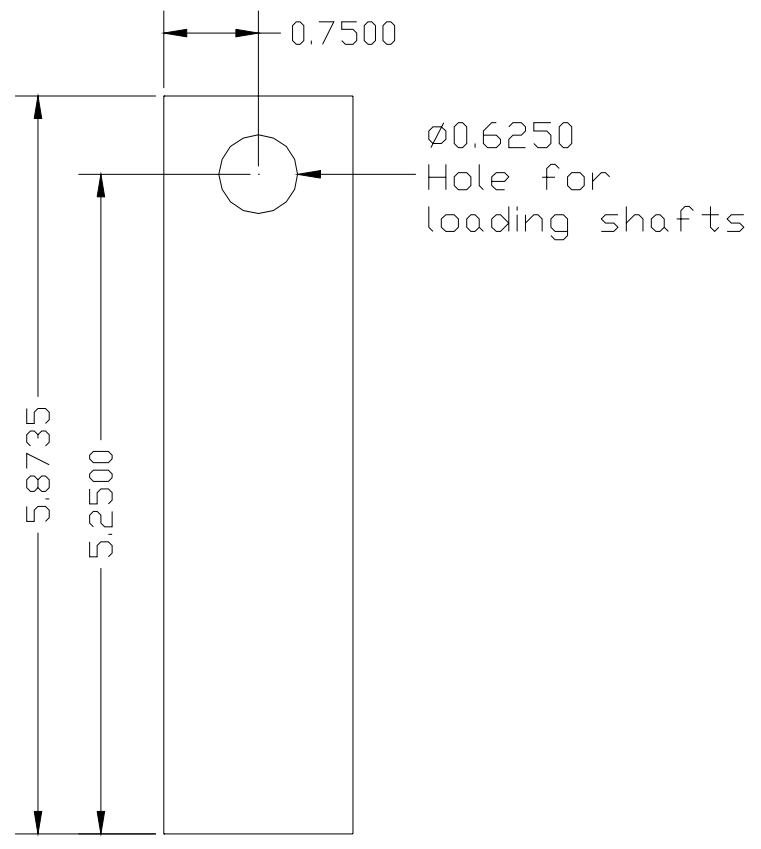

Figure A2 Testing fixture load support arm side view

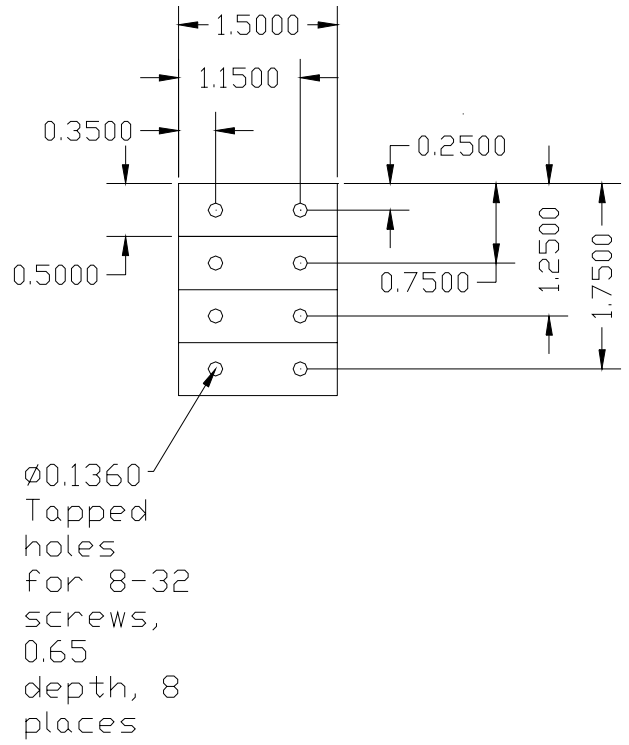

Figure A3 Testing fixture load support arm bottom view 


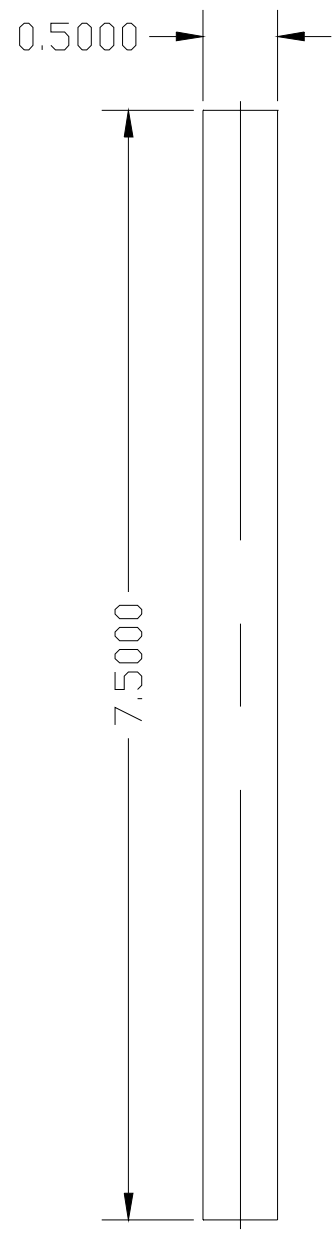

Figure A4 Test fixture ball bearing guide 


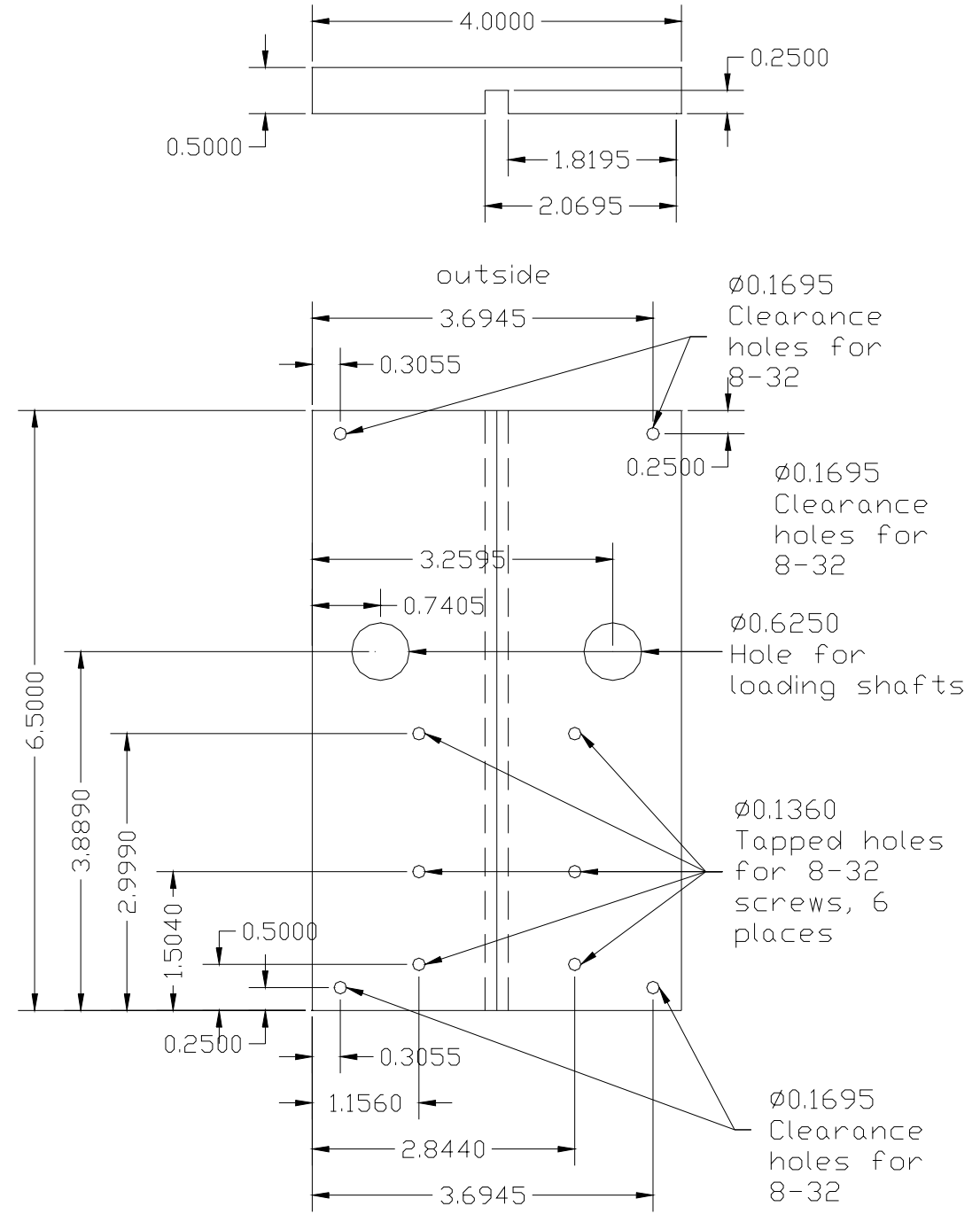

Figure A5 Test fixture carriage assembly top and outside views 


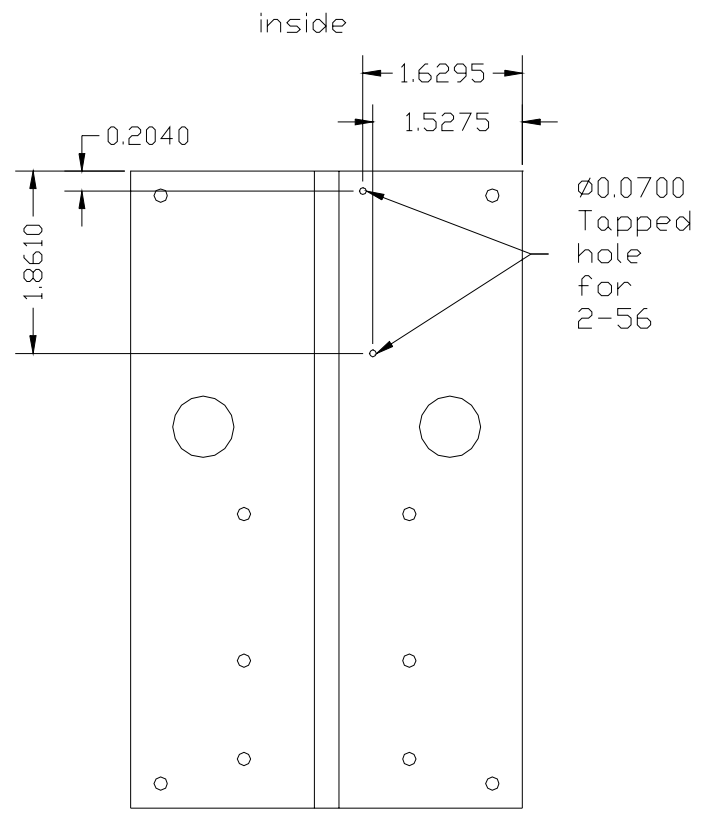

Figure A6 Testing fixture carriage assembly inside view

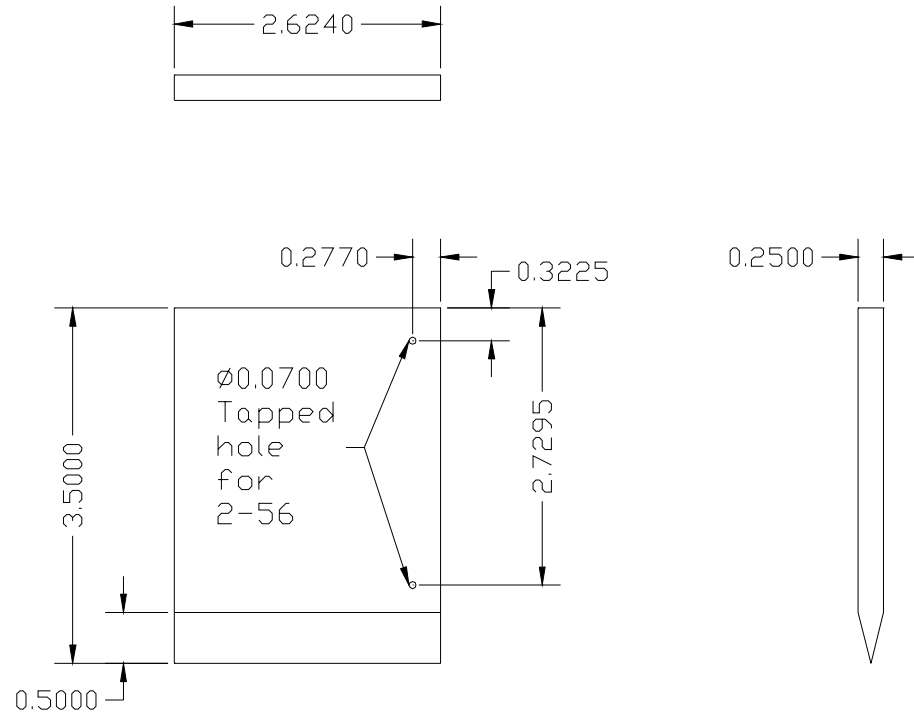

Figure A7 Testing fixture spar and mirror measuring device 


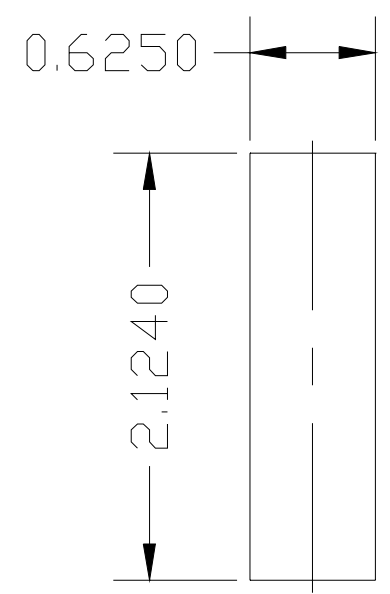

Figure A8 Testing fixture load support 


\section{Appendix B - Software Package}

Visual Basic Code for the form frmSelections

This form was added to the drivers that came with the DAQ card, it allowed the user to select the file to write data to and the time interval in which it wrote the data, Fig. B.1 is a screen shot of this form.

Private Sub cmdExit_Click()

Unload F5312

Unload frmSelections

End

End Sub

Private Sub cmdRecord1_Click()

If filenamechange $1=0$ Then

frmChangefilename. Show

Else

Open frmSelections.CommonDialog2.FileName For Output As \#2

Print \#2, Date

Print \#2, Time

AXIS1data $=$ Val(F5312.Text31Val)

Print \#2, Date; Time; " "; Format(AXIS1data, "00.000")

$\mathrm{j}=$ Timeintervalinput

Timer2.Enabled $=$ True

Timer2.Interval $=$ Timeintervalinput $* 1000$

End If

End Sub

Private Sub cmdRecord0_Click()

If filenamechange $0=0$ Then

frmChangefilename. Show

Else

Open frmSelections.CommonDialog1.FileName For Output As \#1

Print \#1, Date

Print \#1, Time

AXIS0data $=$ Val(F5312.Text30Val)

Print \#1, Date; Time; " "; Format(AXIS0data, "00.000")

$\mathrm{i}=$ Timeintervalinput

Timer1.Enabled $=$ True

Timer1.Interval $=$ Timeintervalinput $* 1000$

End If

End Sub

Private Sub cmdRecord2_Click()

If filenamechange $2=0$ Then

frmChangefilename. Show

Else

Open frmSelections.CommonDialog3.FileName For Output As \#3 


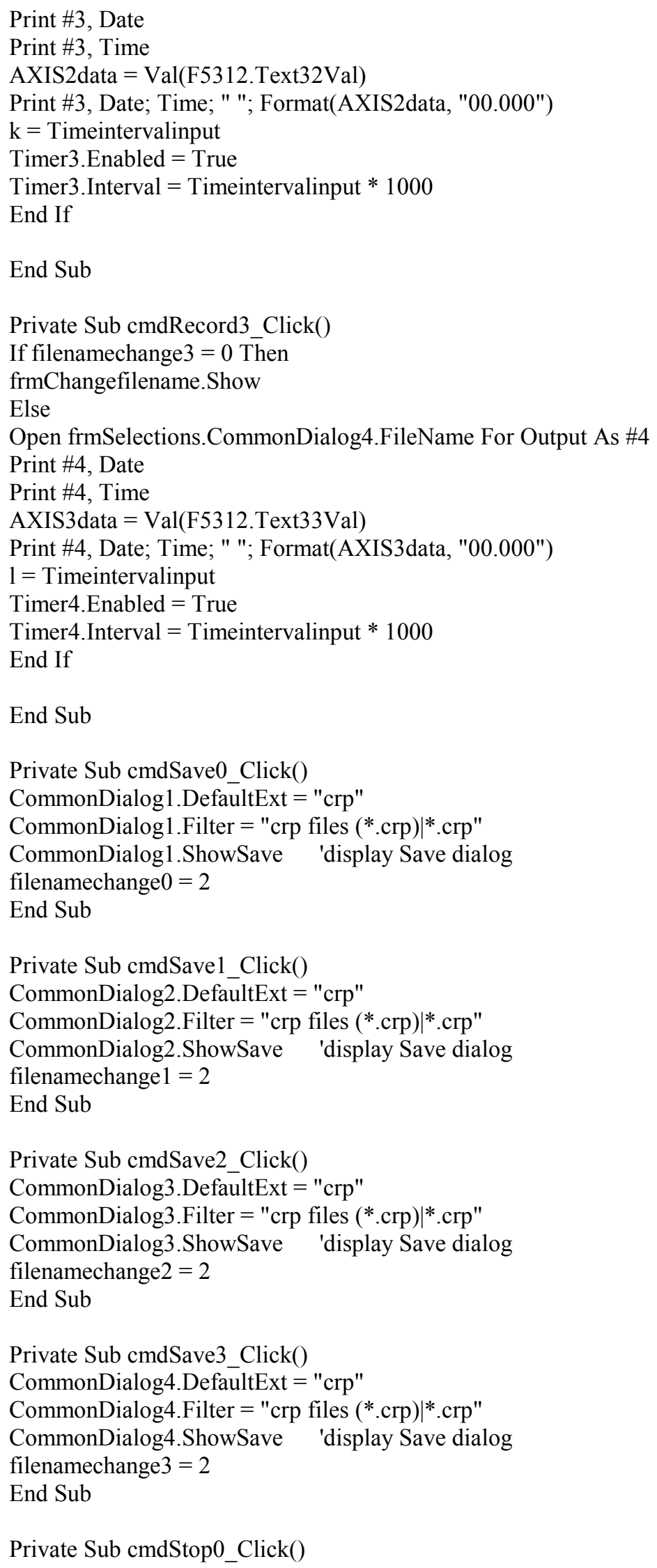


Timer1.Enabled = False
Print \#1, Time
Print \#1, Date
Close \#1
$\mathrm{i}=0$
End Sub
Private Sub cmdStop1_Click()
Timer2.Enabled = False
Print \#2, Time
Print \#2, Date
Close \#2
j=0
End Sub
Private Sub cmdStop2_Click()
Timer3.Enabled = False
Print \#3, Time
Print \#3, Date
Close \#3
$\mathrm{k}=0$
End Sub
Private Sub cmdStop3_Click()
Timer4.Enabled = False
Print \#4, Time
Print \#4, Date
Close \#4
$1=0$
End Sub

Private Sub Form_Load()

filenamechange $0=0$

filenamechange $1=0$

filenamechange $2=0$

filenamechange $3=0$

End Sub

Private Sub ReadCmd_Click()

Dim ret1 As Integer

Dim ResetTemp As Long

'--Reload the counter reset all register's for all Axis.

ret1 $=$ te5312LoadCntr $(0,0)$

ResetTemp $=$ te 5312ReadCntr $(0)$

'Text1 Val.Text $=$ ResetTemp

'Text2Val.Text $=$ Hex(ResetTemp)

ret1 $=$ te5312WriteCmd(-1, TE5312MCR_FLAG_RST)

ret $1=$ te5312WriteCmd(-1, TE5312MCR_CMP_RST)

End Sub

Private Sub Timer1_Timer()

AXIS0data $=$ Val(F5312.Text30Val $)$

Print \#1, Date; Time; " "; Format(AXIS0data, "00.000") 
$\mathrm{i}=\mathrm{i}+$ Timeintervalinput

End Sub

Private Sub Timer2 Timer()

AXIS1data $=$ Val $($ F5312.Text31 Val $)$

Print \#2, Date; Time; " "; Format(AXIS1data, "00.000")

$\mathrm{j}=\mathrm{j}+$ Timeintervalinput

End Sub

Private Sub Timer3 Timer()

AXIS2data $=$ Val(F5312.Text32 Val $)$

Print \#3, Date; Time; " "; Format(AXIS2data, "00.000")

$\mathrm{k}=\mathrm{k}+$ Timeintervalinput

End Sub

Private Sub Timer4_Timer()

AXIS3data $=$ Val(F5312.Text33Val)

Print \#4, Date; Time; " "; Format(AXIS3data, "00.000")

$1=1+$ Timeintervalinput

End Sub

Private Sub txtTimeinterval Change()

Timeintervalinput $=\operatorname{Val}(\operatorname{txt} \bar{T}$ imeinterval $)$

End Sub 
Visual Basic code for the form frmF5312

This code was modified so that the driver recorded in quadrature mode at all times. It is used to zero the system and allows the user to get to the frmSelections file, Fig. B.2 is a screen shot of this form.

Private Sub BrdAdr_Click()

Addr.Show

End Sub

Private Sub cmdRecordData Click()

If filenamechange $=1$ Then

frmChangefilename.Show

End If

If filenamechange $=2$ Then

'---Stop reading the counter.

Timer1.Enabled $=$ False

'---If "Don't show dialog" is not checked, show it.

If Demo1Chk $=$ False Then

Demo1Dlg. Show 1

End If

'---Write the registers for Quadrature operation argument $2=$ TE5312CMD_ICR Or TE5312ICR_ENABLE Or TE5312ICR_LATCH

retval $=$ te $5312 \mathrm{WriteCmd}(\overline{\mathrm{ALLAXIS}}$, argument 2$)$

argument 2 = TE5312CMD_OCCR Or OCCR_Qrd

retval $=$ te $5312 \mathrm{Write} \mathrm{Cmd}(\overline{\mathrm{ALLAXIS}}, \operatorname{argument} 2)$

'---If no dialog box appears, initial count is 0

If Demo1Chk = True Then

retval $=$ te5312LoadCntr(AXIS0, 0)

retval $=$ te5312LoadCntr(AXIS1, 0)

retval $=$ te5312LoadCntr(AXIS2, 0)

retval $=$ te5312LoadCntr(AXIS3, 0)

End If

Text29Val.Text $=$ Demo1String

'---Start reading the counter.

Timer1.Enabled $=$ True

Graph.Show

End If

End Sub 
Private Sub cmdSaveAs_Click()

CommonDialog1.DefaultExt = "dat"

CommonDialog1.Filter $=$ "dat files $\left.\left({ }^{*}\right.$.dat $)\right|^{*}$.dat"

CommonDialog1.ShowSave 'display Save dialog

filenamechange $=2$

End Sub

Private Sub cmdSelections_Click()

'---Stop reading the counter.

Timer1.Enabled $=$ False

'---If "Don't show dialog" is not checked, show it.

If Demo1Chk $=$ False Then

Demo1Dlg. Show 1

End If

'---Write the registers for Quadrature operation

argument $2=$ TE5312CMD_ICR Or TE5312ICR_ENABLE Or TE5312ICR_LATCH

retval $=$ te5312 WriteCmd(ALLAXIS, argument 2$)$

argument2 = TE5312CMD_OCCR Or OCCR_Qrd

retval $=$ te $5312 \mathrm{WriteCmd}(\overline{\mathrm{A}} L \mathrm{~L}$ AXIS, $\operatorname{argument} 2)$

'---If no dialog box appears, initial count is 0

If Demo1Chk $=$ True Then

retval $=$ te5312LoadCntr(AXIS0, 0)

retval $=$ te5312LoadCntr(AXIS1, 0)

retval $=$ te5312LoadCntr(AXIS2, 0)

retval $=$ te5312LoadCntr(AXIS3, 0)

End If

Text29Val.Text $=$ Demo1String

'---Start reading the counter.

Timer1.Enabled $=$ True

F5312.Hide

frmSelections. Show

End Sub

Private Sub cmdTime_Click()

Unload frmSelection

frmSelection. Show

End Sub

Private Sub Command1_Click()

Form1.Show

End Sub

Private Sub Demo1_Click()

"'This is what we are using!!"'

'---Stop reading the counter.

Timer1.Enabled $=$ False

'---If "Don't show dialog" is not checked, show it.

If Demo1Chk $=$ False Then 


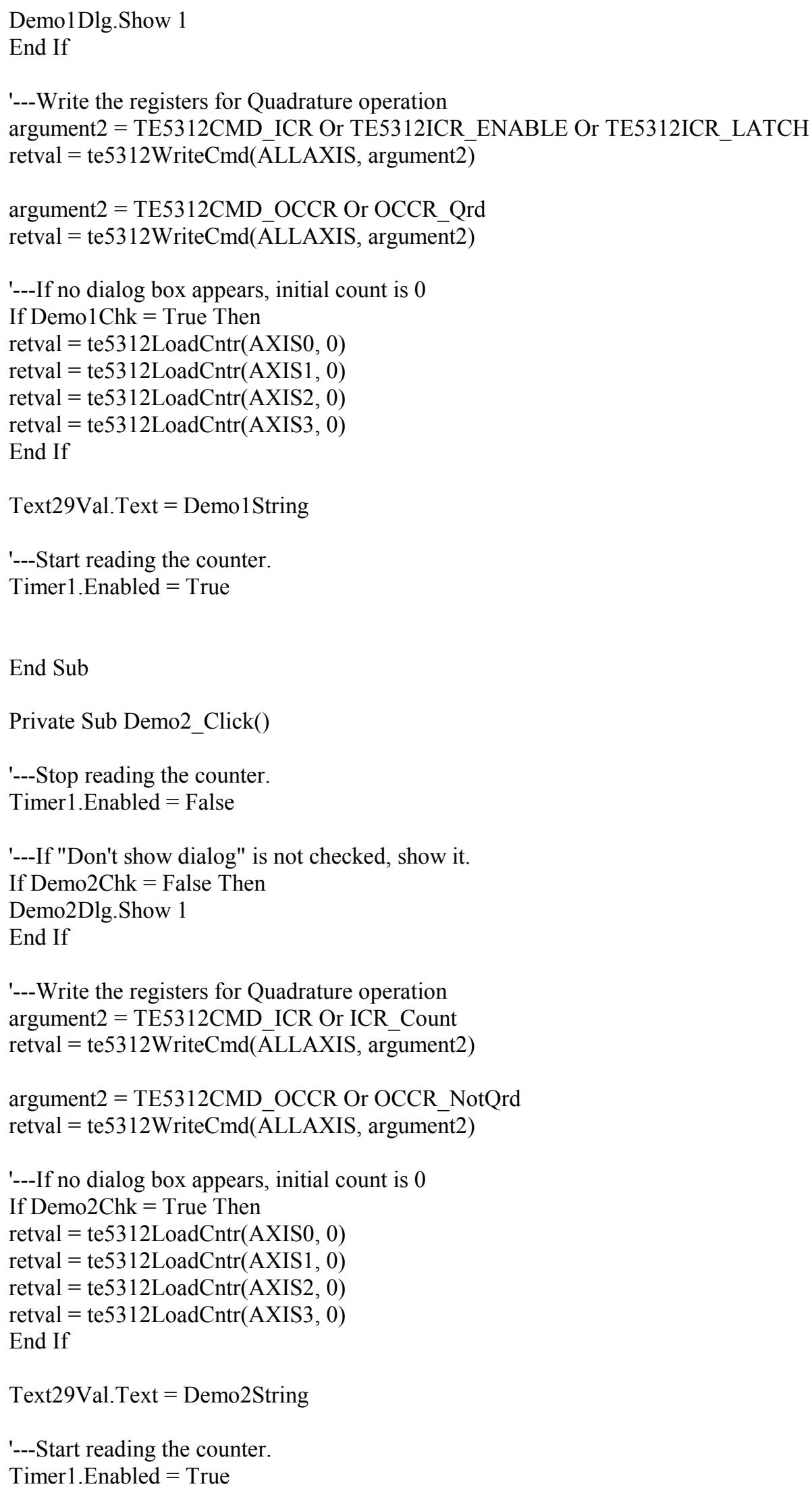


End Sub

Private Sub Demo3_Click()

'---Stop reading the counter.

Timer1.Enabled $=$ False

'---If "Don't show dialog" is not checked, show it.

If Demo3Chk $=$ False Then

Demo3Dlg.Show 1

End If

'---Write the registers for Quadrature operation argument2 = TE5312CMD_ICR Or ICR_Quad retval $=$ te 5312WriteCmd(ALLAXIS, argument2)

argument $2=$ TE5312CMD_OCCR Or OCCR_NotQrd

retval $=$ te $5312 \mathrm{WriteCmd}(\overline{\mathrm{A}} \mathrm{LLAXIS}$, argument 2$)$

'---If no dialog box appears, initial count is 0

If Demo3Chk = True Then

retval $=$ te5312 LoadCntr(AXIS0, 0)

retval $=$ te5312LoadCntr(AXIS1, 0)

retval $=$ te5312LoadCntr(AXIS2, 0)

retval $=$ te5312LoadCntr(AXIS3, 0)

End If

Text29Val.Text $=$ Demo3String

'---Start reading the counter.

Timer1.Enabled $=$ True

End Sub

Private Sub Demo4_Click()

'---Stop reading the counter.

Timer1.Enabled $=$ False

'---If "Don't show dialog" is not checked, show it.

If Demo4Chk $=$ False Then

Demo4Dlg.Show 1

End If

'---Write the registers for Quadrature operation argument2 = TE5312CMD_ICR Or ICR_Quad retval $=$ te5312WriteCmd(ALLAXIS, argument2)

argument $2=$ TE5312CMD_OCCR Or OCCR_Qrd retval $=$ te5312 WriteCmd(ALLAXIS, argument2)

'---If no dialog box appears, initial count is 0

If Demo4Chk = True Then

retval $=$ te5312LoadCntr(AXIS0, 0)

retval $=$ te5312LoadCntr(AXIS1, 0)

retval $=$ te5312LoadCntr(AXIS2, 0) 


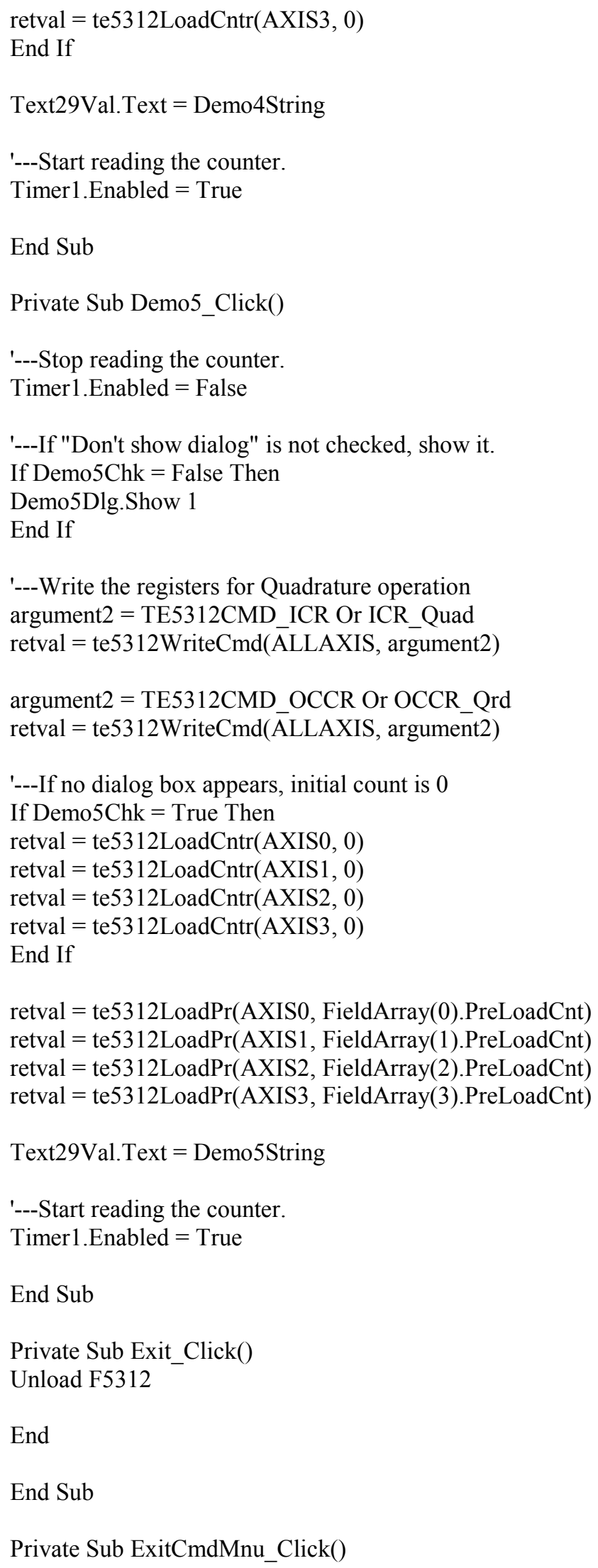


Unload F5312

End

End Sub

Private Sub Form_Unload(Cancel As Integer)

retval $=$ CloseTheDriver ()

End Sub

Private Sub Form_Load()

'Lengthoftest $=60$

'Timeinterval $=1$

filenamechange $=1$

End Sub

Private Sub ReadCmd_Click()

Dim ret1 As Integer

Dim ResetTemp As Long

'--Reload the counter reset all register's for all Axis.

ret1 $=$ te 5312LoadCntr $(0,0)$

ResetTemp $=$ te 5312ReadCntr(0)

Text 1 Val.Text $=$ ResetTemp

Text2Val.Text $=$ Hex $($ ResetTemp $)$

ret $1=$ te5312WriteCmd $(-1$, TE5312MCR_FLAG_RST)

ret $1=$ te5312WriteCmd(-1, TE5312MCR_CMP_RST)

End Sub

Private Sub ResetAllMnu_Click()

Dim ret1 As Integer

Dim ResetTemp As Long

'--Reload the counter reset all register's for all Axis.

ret1 $=$ te 5312 LoadCntr $(0,0)$

ResetTemp $=$ te 5312ReadCntr(0)

Text 1 Val.Text $=$ ResetTemp

Text2Val.Text $=$ Hex $($ ResetTemp $)$

ret1 $=$ te5312WriteCmd(-1, TE5312MCR_FLAG_RST)

ret $1=$ te5312WriteCmd(-1, TE5312MCR_CMP_RST)

End Sub

Private Sub SaveAsCmdMnu_Click()

CommonDialog1.DefaultExt $=$ "dat"

CommonDialog1.Filter $=$ "dat files $\left.(*$.dat $)\right|^{*}$.dat"

CommonDialog1.ShowSave 'display Save dialog

End Sub

Private Sub ShwDlgMnu_Click()

Demo1Dlg.Check1. Value $=0$

Demo1Chk $=$ False

Demo1Dlg.Check1. Value $=0$ 


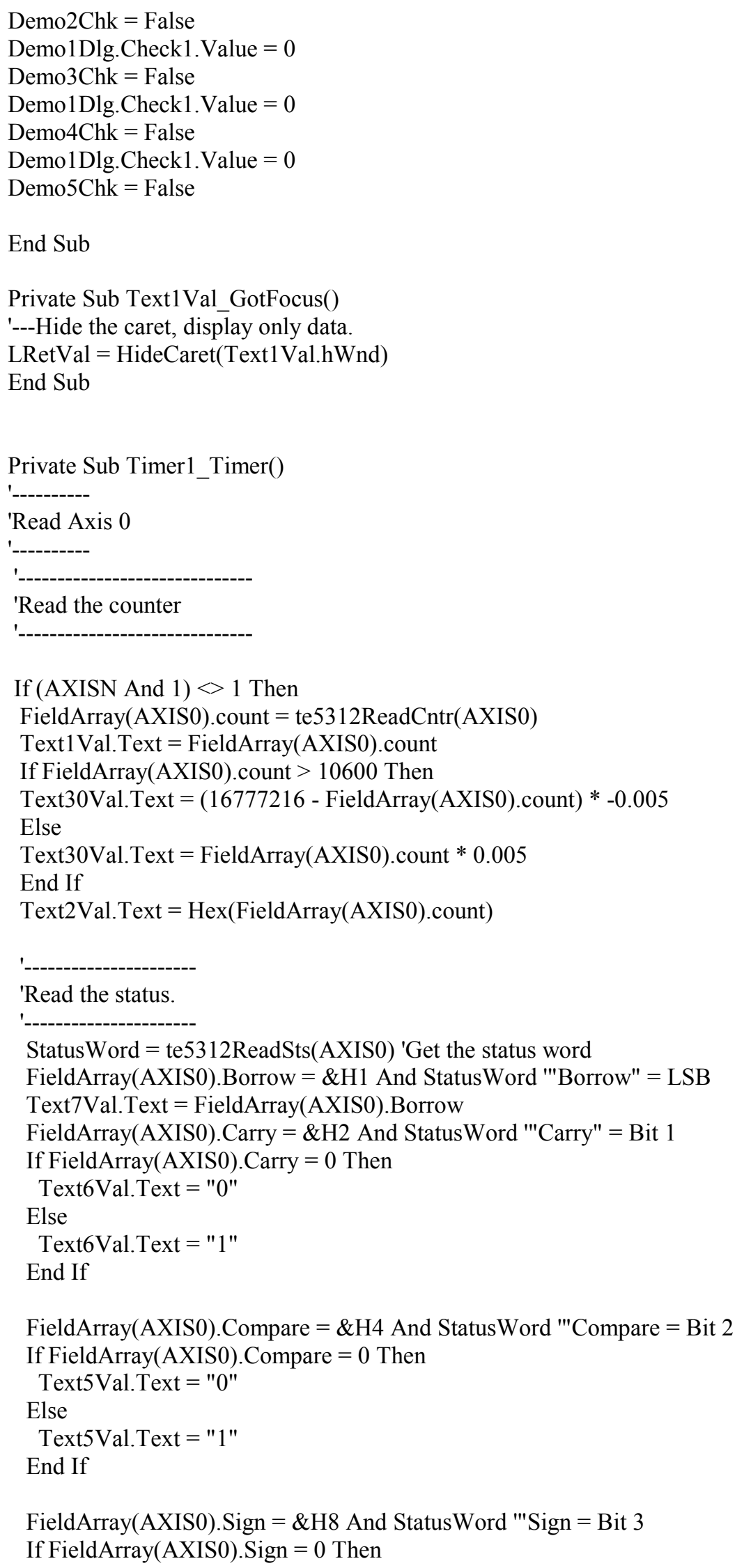




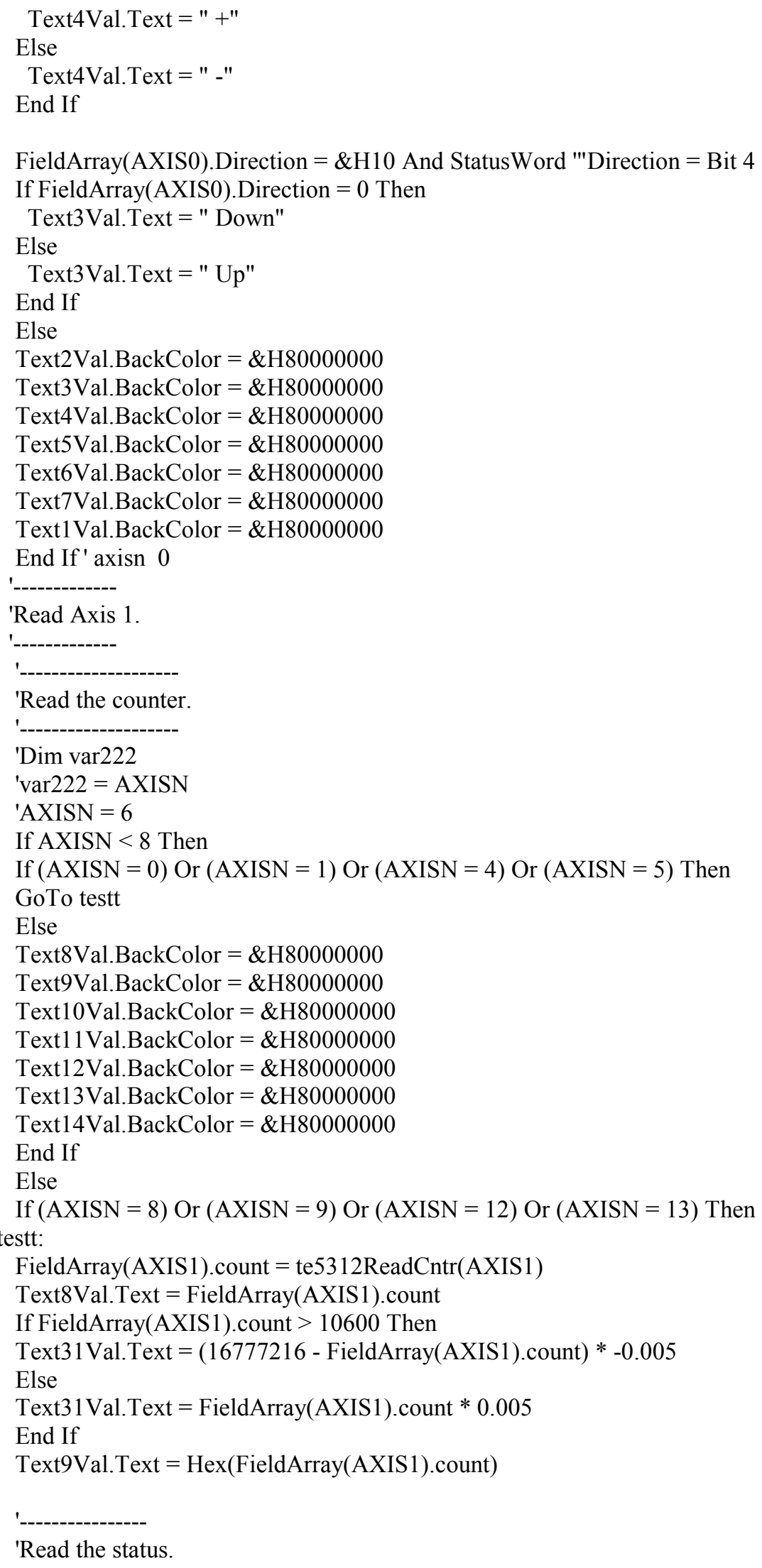


StatusWord = te5312ReadSts(1) 'Get the status word

FieldArray(AXIS1). Borrow $=$ \&H1 And StatusWord "'Borrow" = LSB

Text14Val.Text $=$ FieldArray(AXIS1).Borrow

FieldArray(AXIS1).Carry = \&H2 And StatusWord "'Carry" = Bit 1

If FieldArray(AXIS1).Carry $=0$ Then

Text13Val.Text $=$ "0"

Else

Text13Val.Text $=" 1 "$

End If

FieldArray(AXIS1).Compare = \&H4 And StatusWord "'Compare = Bit 2

If FieldArray(AXIS1).Compare $=0$ Then

Text12Val.Text = "0"

Else

Text12Val.Text $=" 1 "$

End If

FieldArray(AXIS1).Sign = \&H8 And StatusWord "'Sign = Bit 3

If FieldArray(AXIS1).Sign $=0$ Then

Text11Val.Text $="+"$

Else

Text11Val.Text = " -"

End If

FieldArray(AXIS1).Direction $=\&$ H10 And StatusWord "'Direction $=$ Bit 4

If FieldArray(AXIS1).Direction $=0$ Then

Text10Val.Text $=$ " Down"

Else

Text10Val.Text $=$ " Up"

End If

Else

Text8Val.BackColor $=\& H 80000000$

Text9Val.BackColor $=\& H 80000000$

Text10Val.BackColor $=\& H 80000000$

Text11 Val.BackColor $=\& H 80000000$

Text12Val.BackColor $=\& H 80000000$

Text13Val.BackColor $=\& H 80000000$

Text14Val.BackColor $=\& H 80000000$

End If

End If ' Axisn 1

'Read Axis 2.

'-

'Read the counter.

'Dim var222

'var222 = AXISN

'AXISN $=10$

If AXISN $<8$ Then

If $\mathrm{AXISN}=0$ Or AXISN $=1$ Or $\mathrm{AXISN}=2$ Or $\mathrm{AXISN}=3$ Then

GoTo tt

Else

Text15Val.BackColor $=\& H 80000000$ 


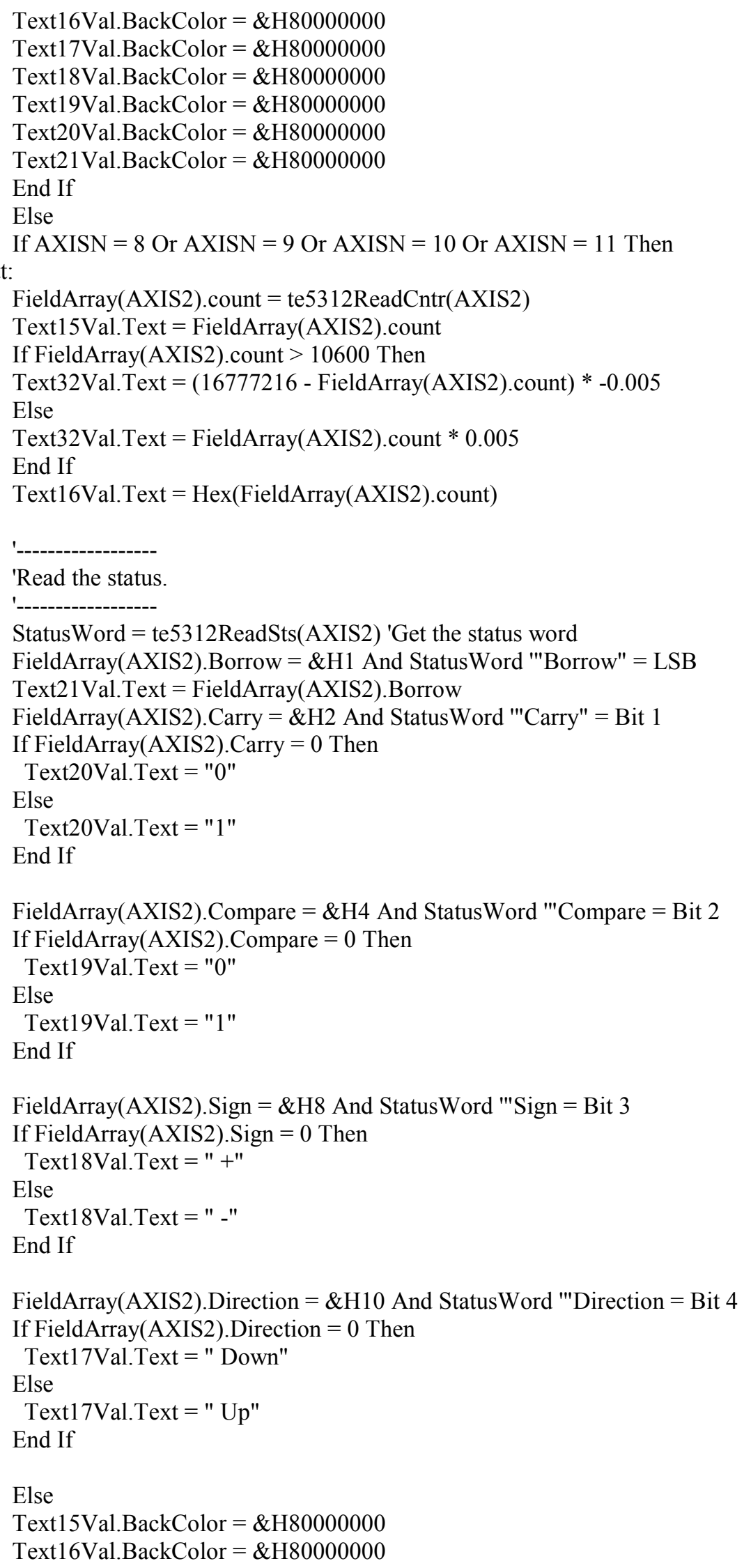




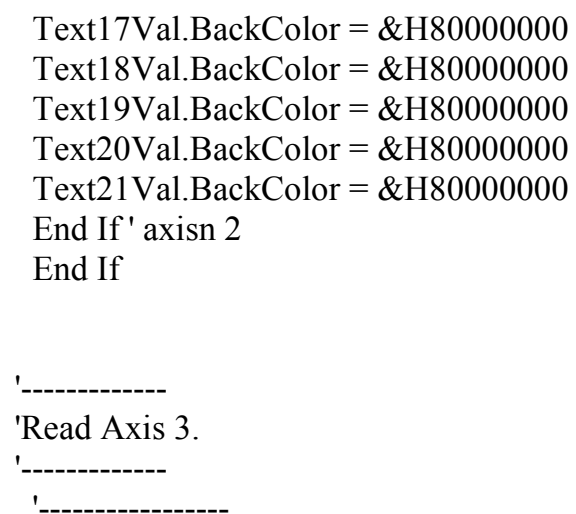

FieldArray(AXIS3).Direction $=\& H 10$ And StatusWord "'Direction $=$ Bit 4 If FieldArray(AXIS3).Direction $=0$ Then Text24Val.Text = " Down" 


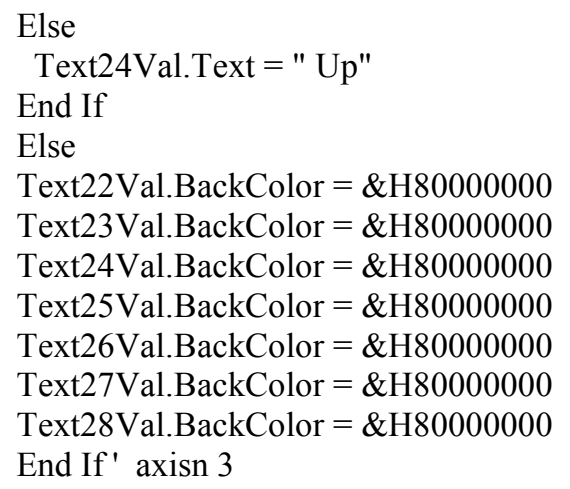

End Sub

Private Sub TimeSelectMnu_Click()

frmSelection. Show

End Sub 
Visual Basic code for the module variable

This module was added to the original code, it contains defines all of the variables that were added to the software.

Public testvar As Integer

Public AXIS0data!

Public AXIS1data!

Public AXIS2data!

Public AXIS3data!

Public graphx() As Double Public graphy1() As Double Public graphy2() As Double Public graphy3() As Double

Public graphy4() As Double

Public selec As Integer

Public testcheck As Integer

Public timecheck As Integer

Public filenamechange0 As Integer

Public filenamechange1 As Integer

Public filenamechange2 As Integer

Public filenamechange3 As Integer

Public Lengthoftestinput!

Public Timeintervalinput!

Public Lengthoftest!

Public Timeinterval!

Public i As Long

Public $\mathrm{j}$ As Long

Public k As Long

Public 1 As Long

Public counterp

Public AXIS0datap

Public displacement

Public c0 As Integer

Public c1 As Integer

Public c2 As Integer

Public c3 As Integer

Public 10!

Public 11!

Public 12!

Public 13! 


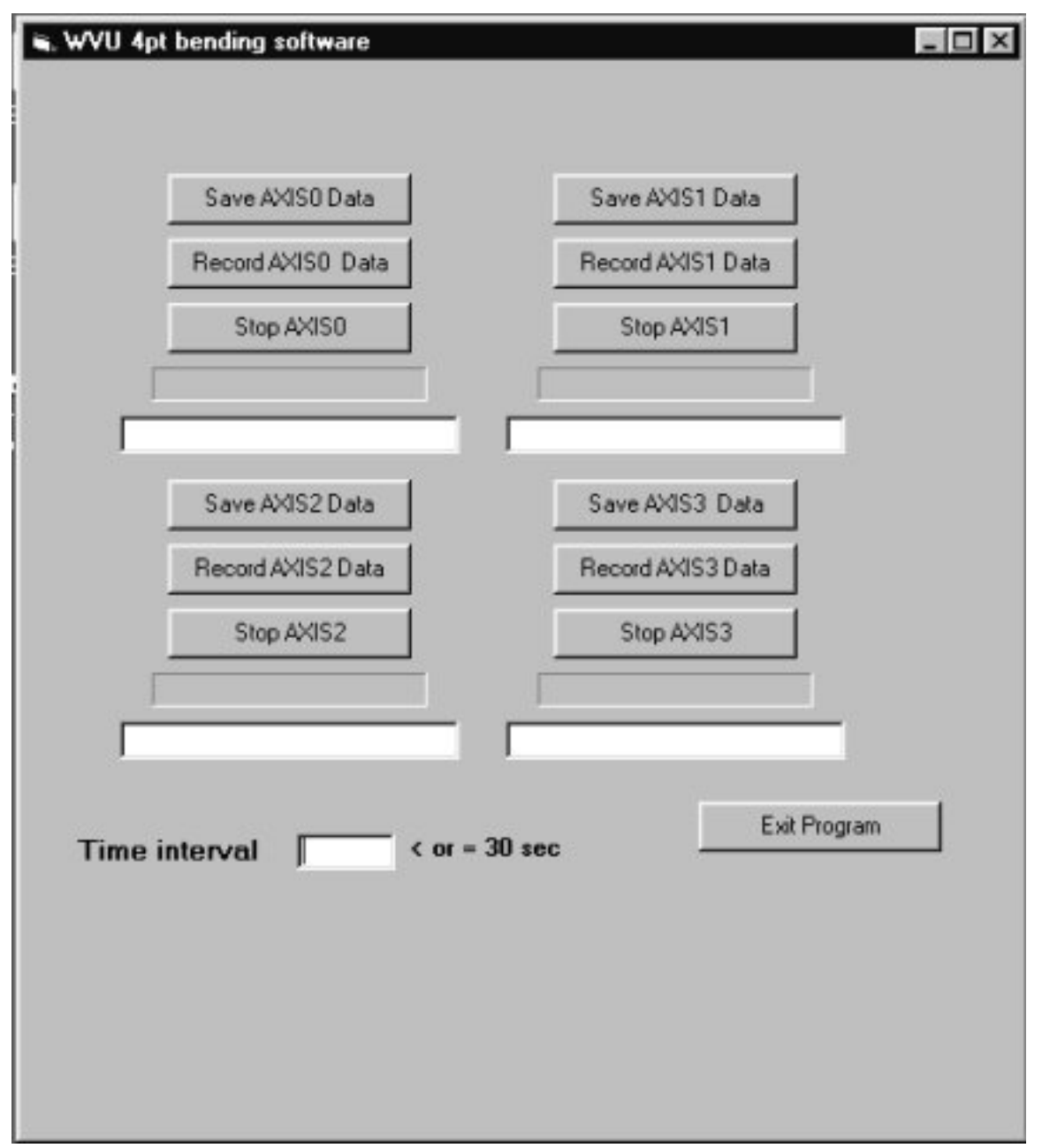

Figure B.1 Screen shot of the form frmSelections, which allows users to enter the filename to write to and select the time interval to write the data. 


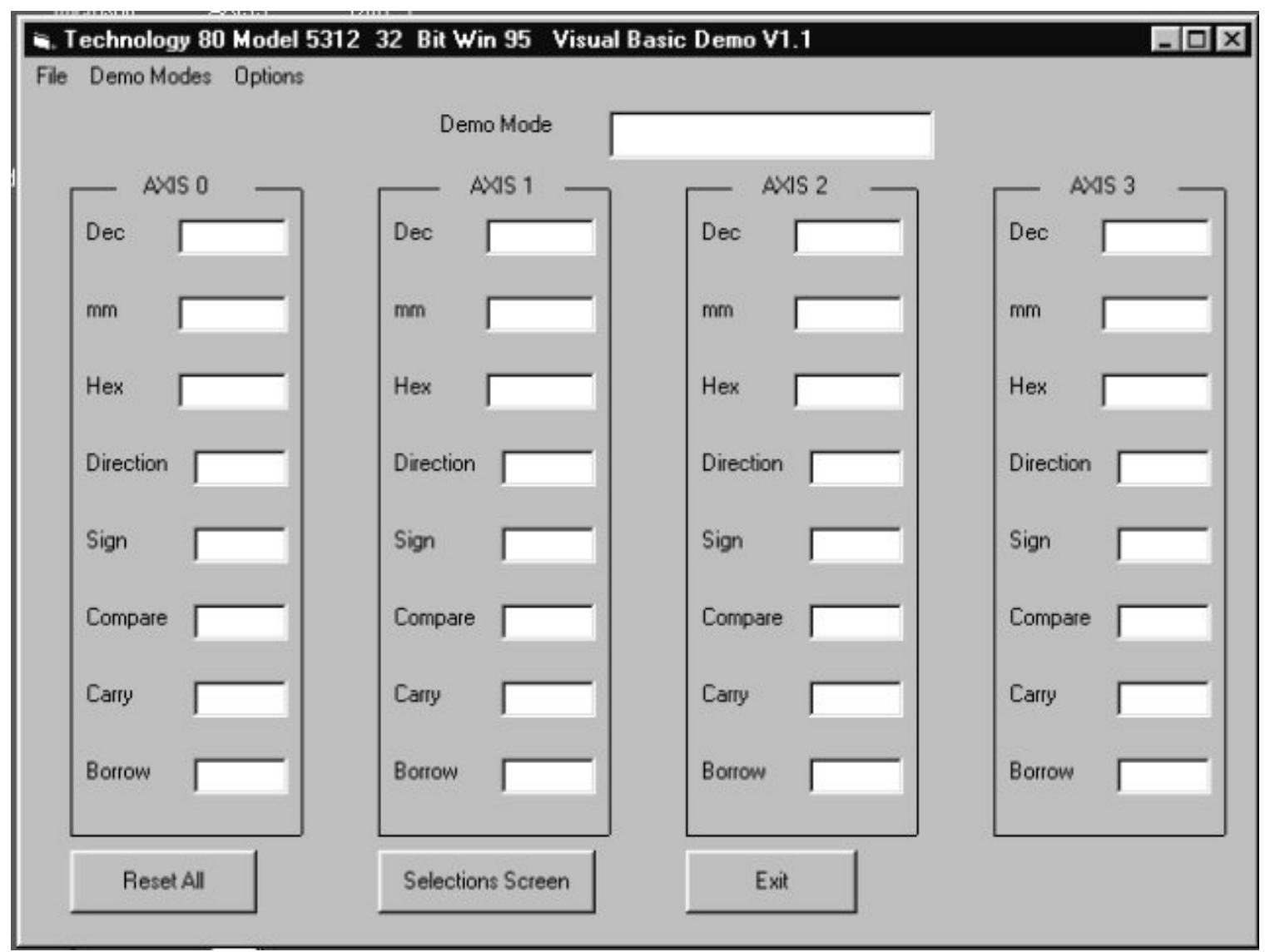

Figure B.2 Screen shot of the from frmF5312 which allows users to zero the system. 